\title{
Directional Derivatives of Oblique Reflection Maps
}

\author{
Avishai Mandelbaum \\ School of Industrial Engineering and Management \\ Technion, Haifa, Israel \\ and \\ Kavita Ramanan* \\ Department of Mathematical Sciences \\ Carnegie Mellon University, Pittsburgh, PA
}

\begin{abstract}
Given an oblique reflection map $\Gamma$ and functions $\psi, \chi \in \mathcal{D}_{\lim }$ (the space of $\mathbb{R}^{K}$-valued functions that have left and right limits at every point), the directional derivative $\nabla_{\chi} \Gamma(\psi)$ of $\Gamma$ along $\chi$, evaluated at $\psi$, is defined to be the pointwise limit (as $\varepsilon \downarrow 0$ ) of the family of functions $\nabla_{\chi}^{\varepsilon} \Gamma(\psi) \doteq \varepsilon^{-1}[\Gamma(\psi+\varepsilon \chi)-\Gamma(\psi)]$. Directional derivatives are shown to exist and lie in $\mathcal{D}_{\lim }$ for oblique reflection maps associated with reflection matrices of the so-called Harrison-Reiman class. When $\psi$ and $\chi$ are continuous, the convergence of $\nabla_{\chi}^{\varepsilon} \Gamma(\psi)$ to $\nabla_{\chi} \Gamma(\psi)$ is shown to be uniform on compact subsets of continuity points of the limit $\nabla_{\chi} \Gamma(\psi)$ and the derivative $\nabla_{\chi} \Gamma(\psi)$ is shown to have an autonomous characterization as the unique fixed point of an associated map. Motivation for the study of directional derivatives stems from the fact that they arise as functional central limit approximations to time-inhomogeneous queueing networks as well as transient time-homogeneous queueing networks. This work also shows how the various types of discontinuities of the derivative $\nabla_{\chi} \Gamma(\psi)$ are related to the reflection matrix and properties of the function $\Gamma(\psi)$. In the queueing network context, this describes the influence of the topology of the network and the states (of underloading, overloading or criticality) of the various queues in the network on the discontinuities of $\nabla_{\chi} \Gamma(\psi)$.
\end{abstract}

Key words. Reflection map, Skorokhod Map, time-inhomogeneous queueing networks, non-

${ }^{*}$ This material is based upon work partially supported by the National Science Foundation under Grants 0405343, 0406191 and 0323668-0000000965 
stationarity, time-varying rates, transient behavior, periodic queues, fluid limits, diffusion approximations, heavy traffic, directional derivatives, overloading, underloading, criticality. 


\section{Contents}

1 Introduction $\quad 5$

1.1 Motivation and Outline of the Paper . . . . . . . . . . . . 5

1.2 Basic Notation . . . . . . . . . . . . . . . . . . . 7

1.3 Definition of the Directional Derivative . . . . . . . . . . . . . . . . 9

1.4 Main Results of the Paper . . . . . . . . . . . . . . . . . . . . . 11

1.4.1 Existence of the derivative . . . . . . . . . . . . . . . . 11

1.4.2 Discontinuities of the derivative $\nabla_{\chi} \Gamma(\psi)$ for continuous $\psi, \chi \ldots \ldots 14$

2 Connection with Queueing Networks $\quad 17$

2.1 Directional Derivatives and Functional Central Limits . . . . . . . . . . . . 17

2.2 Illustrative Examples . . . . . . . . . . . . . . . . . . . . . . 19

2.2 .1 A tandem queueing model . . . . . . . . . . . . . . . . 20

2.2 .2 A merge or join network . . . . . . . . . . . . . . . . . . . 22

3 Existence and Characterization of the Directional Derivative 24

3.1 Properties of the Oblique Reflection Map . . . . . . . . . . . . . . . . . . 25

3.2 Existence of the Directional Derivative . . . . . . . . . . . . . . . . 26

3.2 .1 A related sequence $\left\{\gamma_{\varepsilon}\right\} \ldots \ldots \ldots \ldots \ldots$

3.2.2 Pointwise convergence of $\left\{\gamma_{\varepsilon}\right\}$ for H-R ORPs . . . . . . . . . 27

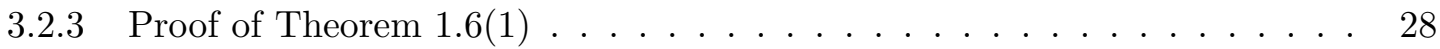

3.3 The Generalized One-dimensional Derivative . . . . . . . . . . . . . . 30

3.3.1 A representation for $\gamma^{i} \ldots \ldots \ldots \ldots \ldots \ldots$

3.3.2 Identification of the generalized one-dimensional derivative . . . . . . . 31

3.4 Proof of the First Main Result . . . . . . . . . . . . . . . . . . . . 34

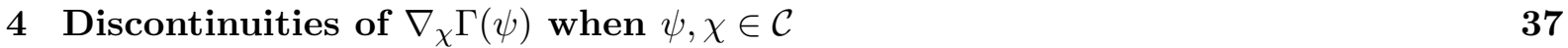

4.1 Classification of the Discontinuities of $\gamma_{(1)} \ldots \ldots \ldots \ldots \ldots$

4.2 Alternative Description of the Regimes of $(\phi, \theta) \ldots \ldots \ldots$. . . . . . . 43

4.3 Proof of the Second Main Result . . . . . . . . . . . . . . . . . . . . . . . 44 
5 Proof of Theorem 3.5

6 Auxiliary Results 


\section{Introduction}

\subsection{Motivation and Outline of the Paper}

Most real-world queueing systems are time-inhomogeneous in the sense that they evolve according to transition laws that themselves vary with time. However, the majority of queueing research has been devoted to time-homogeneous models, in which the transition laws are assumed to be independent of time. While such models may provide reasonable approximations for slowly varying systems, they completely fail to capture many important phenomena such as surges in demand, sudden node failures and periodicity. The explicit analysis of even time-homogeneous networks is usually intractable. Instead, one usually resorts to appropriate asymptotic approximations that capture the essential features of network behavior that are of interest. A commonly used asymptotic scaling is one in which arrival and service rates are scaled proportionately, but the number of servers at each queue is kept constant. Over the past two decades, much progress has been made on this kind of approximation for time-homogeneous networks with fairly general arrival, service and routing processes that satisfy a so-called heavy-traffic condition. In particular, under an additional initial assumption on the queues that guarantees that the first-order asymptotic limit (or fluid limit) is trivially zero, it has been shown that the second-order asymptotic limits associated with various classes of of time-homogeneous queueing networks are reflected Brownian motions (RBMs) or reflected Lévy processes (see, for example, [6, 7, 8, 21, 37, 41, 40, 51, 49] and references therein). In contrast, the analysis of time-inhomogeneous networks remains challenging even in a Markovian setting. In particular, there has been relatively little work done on second-order approximations to time-inhomogeneous queueing networks with a fixed number of servers. Such networks arise frequently as models of transportation, telecommunication and computer systems (see, for example, [11, 23, 34]).

The single queue with time-varying arrival and service rates has been studied by various authors under different assumptions [17, 25, 31, 32, 42, 43]. The detailed asymptotic analysis carried out in [25] is pathwise and uses strong approximations. It shows that the so-called fluid limit or first-order approximation of a time-dependent Markovian queue alternates between phases of overloading, critical loading and underloading and that the second-order correction to the fluid limit exhibits different characteristics in each of the three different phases of loading. This second-order correction admits an interpretation as the directional derivative of the one- 
dimensional reflection map $\Gamma$. In subsequent work [39], we show that such an interpretation continues to hold in the network setting, in the sense that the corresponding second-order corrections in the asymptotic approximations to a class of time-inhomogeneous networks can be characterized as directional-derivatives of associated multi-dimensional reflection maps (also see Section 2.1 for a formal discussion of this connection). With this as a motivation, the main objectives of this work are to introduce and characterize properties of directional derivatives of the class of so-called Harrison-Reiman multi-dimensional reflection maps (which are associated with single-class open queueing networks), and to illustrate the practical insights that can be obtained from such an analysis.

The representation obtained in [25] for the directional derivative of the one-dimensional reflection map $\Gamma$ relied heavily on the following explicit form for $\Gamma$ due to [46]:

$$
\Gamma(\psi)(t)=\psi(t)+\theta(t)
$$

for càdlàg functions $\psi$, where the constraining term $\theta$ that keeps $\Gamma(\psi)$ non-negative is given by

$$
\theta(t)=\max \left(\sup _{0 \leq s \leq t}[-\psi(s)], 0\right) .
$$

In contrast, in the multi-dimensional setting there is no explicit expression for the oblique reflection map, making characterization of its directional derivatives considerably more involved. In fact derivatives of reflection maps associated with even feedforward tandem networks cannot always be expressed simply as a composition of directional derivatives of one-dimensional reflection maps (see Section 3.3.2 for further discussion of this fact). The network setting also introduces additional complications due to dependence on network topology and leads to interesting new questions about when and how effects propagate through the network. Consequently new techniques need to be developed to analyze derivatives in the multi-dimensional setting.

The outline of the rest of the paper is as follows. The basic notation used throughout the paper is first collected in Section 1.2. In Section 1.3 the definitions of the multi-dimensional oblique reflection map as well as the Harrison-Reiman class of maps are stated and then the definition of the directional derivative is introduced. The main results of the paper, Theorems 1.6 and 1.10, are presented in Section 1.4. Theorem 1.6 establishes the existence of directional derivatives $\nabla_{\chi} \Gamma(\psi)$ for Harrison-Reiman reflection maps and, under additional conditions on $\psi, \chi$, also provides autonomous characterizations of the derivative. Theorem 1.10 derives necessary conditions for the existence of discontinuities in the directional derivative when $\psi, \chi$ are 
continuous. Section 2 contains a brief discussion of the connection between approximations to time-inhomogeneous queueing networks and directional derivatives of multi-dimensional reflection maps. It also presents illustrative examples that show that the directional derivative can be explicitly calculated in many cases and demonstrate some interesting features that arise in the multi-dimensional or network context. The rest of the paper is essentially devoted to proving the two main results. General properties of Harrison-Reiman maps are summarized in Section 3.1 and existence of the directional derivative is established in Section 3.2, with the proof of Theorem 1.6 presented in Section 3.4. An important ingredient of this proof is the notion of a generalized one-dimensional derivative, which is introduced in Section 3.3, and the representation of the one-dimensional derivative obtained in Theorem 3.5, whose proof is deferred to Section 5. In Section 4 the discontinuities of the directional derivative are analyzed when $\psi, \chi$ are continuous, culminating in the proof of Theorem 1.10 in Section 4.3. The paper concludes with Section 6, which comprises of lemmas establishing general properties of functions that are required for various proofs.

\subsection{Basic Notation}

In this section, for convenience, we compile all the common notation used throughout the paper. For $a, b \in \mathbb{R}$, let $a \vee b=\max (a, b)$ and $a \wedge b=\min (a, b)$. Given a vector $x \in \mathbb{R}^{K}, x^{i}$ or $[x]^{i}$ is used to denote the $i$ th component of the vector. For $a \in \mathbb{R}^{K}$, the norm $|a|$ is defined by

$$
|a| \doteq \max _{i=1, \ldots, K}\left|a^{i}\right|
$$

where, for $a^{i} \in \mathbb{R},\left|a^{i}\right|$ denotes the usual Euclidan norm. Given a $K \times K$ matrix $R, R^{T}$ denotes its transpose, $\sigma(R)$ its spectral radius and $R_{i j}$ represents the entry in the $i$ th row and $j$ th column of $R$. The matrix $I$ represents the $K \times K$ identity matrix, and $\left\{e_{i}, i=1, \ldots, K\right\}$ is the standard orthonormal basis in $\mathbb{R}^{K}$. Inequalities of vectors and matrices should be interpreted componentwise. Vectors are always expressed as column vectors. The $K$-dimensional orthant is denoted by $\mathbb{R}_{+}^{K}$ :

$$
\mathbb{R}_{+}^{K} \doteq\left\{x \in \mathbb{R}^{K}: x^{i} \geq 0 \text { for every } i=1, \ldots, K\right\}
$$

The notation $\uparrow(\downarrow)$ is used to denote monotone decreasing (nonincreasing) convergence of a family of real numbers to a limit. We adopt the convention that the infimum and supremum of an empty set are $-\infty$ and $\infty$, respectively. The notation 0 is used to denote both the number zero as well as the identically zero function - the use should be clear from the context. 
Given a function $f$ on $[0, \infty)$ that takes values in $\mathbb{R}^{K}, f^{i}$ denotes the $i$ th coordinate function. For any $\mathbb{R}^{K}$-valued function $f$ and $T<\infty,\|f\|_{T}$ denotes the supremum norm: $\|f\|_{T} \doteq \sup _{s \in[0, T]}|f(s)|$, where $|\cdot|$ is the norm defined above in (1.3). In addition, the notation $\bar{f}$ is used to denote the supremum function:

$$
\bar{f}(t) \doteq \sup _{s \in[0, t]} f(s)
$$

The analysis in this paper involves the use of many different functions spaces, which are summarized in the following table.

$\mathcal{D}_{\lim } \quad$ the space of all functions on $[0, \infty)$ taking values in $\mathbb{R}^{K}$ that have left and right limits for every $t \in[0, \infty)$

$\mathcal{D}_{\lim }^{+} \quad$ the subspace of functions $f \in \mathcal{D}_{\lim }$ with $f(0) \in \mathbb{R}_{+}^{K}$

$\mathcal{D}_{r} \quad$ the subspace of right continuous functions in $\mathcal{D}_{\text {lim }}$

$\mathcal{D}_{\ell, r} \quad$ the subspace of functions that are either right continuous or left continuous at every $t \in[0, \infty)$

$\mathcal{D}_{\text {usc }} \quad$ the subspace of functions in $\mathcal{D}_{\text {lim }}$ such that each coordinate function $f^{i}$ is upper semicontinuous (i.e. $f(t) \geq f(t-) \vee f(t+)$ for every $t \in[0, \infty))$

$\mathcal{D}_{c, \text { lim }}$ the subspace of piecewise constant functions in $\mathcal{D}_{\text {lim }}$ with a finite number of jumps

$\mathcal{D}_{c} \quad$ the subspace of piecewise constant functions in $\mathcal{D}_{r}$ with a finite number of jumps

$\mathcal{I}^{+} \quad$ the subspace of functions in $\mathcal{D}_{\text {lim }}^{+}$such that each coordinate function is nondecreasing

$\mathcal{I}_{0}^{+} \quad$ the subspace of functions $f \in \mathcal{I}^{+}$such that $f(0)=0$

$\mathcal{C} \quad$ the subspace of continuous functions in $\mathcal{D}_{\text {lim }}$

$\mathcal{B V}$ the subspace of functions in $\mathcal{D}_{\lim }$ that have bounded variation on every bounded interval of $[0, \infty)$

For $f \in \mathcal{B V},|f|_{t}$ denotes the total variation norm on $[0, t]$ (with respect to the norm $|\cdot|$ defined in (1.3) on $\left.\mathbb{R}^{K}\right)$. A function $f \in \mathcal{D}_{\text {lim }}$ is said to have a separated discontinuity at a point $t \in[0, \infty)$ if for some $i=1, \ldots, K, f^{i}(t)$ does not lie in the interval created by $f^{i}(t-)$ and $f^{i}(t+)$ : that is,

$$
f^{i}(t) \notin\left[f^{i}(t-) \wedge f^{i}(t+), f^{i}(t-) \vee f^{i}(t+)\right]
$$

For $f \in \mathcal{D}_{\text {lim }}$, let $\operatorname{Disc}(f)$ (respectively $\operatorname{LDisc}(f), R \operatorname{Risc}(f)$ and $S \operatorname{Disc}(f)$ ) denote the set of 
points of discontinuity (respectively left discontinuity, right discontinuity and separated discontinuity) of $f$. Clearly, $\operatorname{Disc}(f)=\operatorname{LDisc}(f) \cup \operatorname{RDisc}(f)$, and for $f \in \mathcal{D}_{\text {usc }}$, it is easy to see that $\operatorname{SDisc}(f)=\operatorname{LDisc}(f) \cap R \operatorname{Disc}(f)$.

The left and right regularizations of any function $g \in \mathcal{D}_{\text {lim }}$, denoted by $g_{l}$ and $g_{r}$ respectively, are defined by

$$
g_{l}(s) \doteq g(s-) \quad \text { and } \quad g_{r}(s) \doteq g(s+)
$$

for $s \in[0, \infty)$. It is easy to see that $g_{l}(s-)=g_{l}(s)=g(s-)$ and $g_{l}(s+)=g(s+)$, and likewise $g_{r}(s+)=g_{r}(s)=g(s+)$ and $g_{r}(s-)=g(s-)$. Thus $g_{l} \in \mathcal{D}_{l}, g_{r} \in \mathcal{D}_{r}$ and

$$
g \in \mathcal{D}_{l} \Rightarrow g_{l}=g, \quad \text { and } \quad g \in \mathcal{D}_{r} \Rightarrow g_{r}=g .
$$

Lastly, given a real-valued function $f$, a point $t \in[0, \infty)$ is said to be a point of strict left increase if there exists $\delta>0$ such that $f(s)<f(t)$ for every $s \in\left[(t-\delta)^{+}, t\right)$, and of strict right increase if there exists $\delta>0$ such that $f(t)<f(s)$ for every $s \in(t, t+\delta]$. Moreover, $f$ is said to be flat to the left (right) of $t$, and is represented by the notation $\Delta f(t-)=0(\Delta f(t+)=0)$, if there exists $\delta>0$ such that $f(s)=f(t)$ for all $s \in(t-\delta, t]$ (for all $s \in[t, t+\delta)$ ). We will also use the shorthand notation $\Delta f(t-) \neq 0(\Delta f(t+) \neq 0)$ to denote the fact that $f$ is not flat to the left (right) of $t$.

\subsection{Definition of the Directional Derivative}

In this section we state the precise definitions of the oblique reflection map and its directional derivatives. Let $R \in \mathbb{R}^{K \times K}$ be a matrix whose $i$ th column is the vector $r_{i}$, which represents the constraint direction on the face $F_{i}=\left\{x \in \mathbb{R}_{+}^{K}: x^{i}=0\right\}$ of the boundary of the non-negative orthant $\mathbb{R}_{+}^{K}$. Roughly speaking, given a trajectory $\psi \in \mathcal{D}_{\text {lim }}$, the oblique reflection problem (ORP) associated with the constraint matrix $R$ defines a constrained version $\phi$ of $\psi$ that is restricted to live in $\mathbb{R}_{+}^{K}$ by a constraining term that pushes along the direction $r_{i}$ only when $\phi$ lies on the face $F_{i}$. We will assume that for every $i=1, \ldots, K, R_{i i}=r_{i}^{i}>0$. This ensures that from any point in the relative interior of the face $F_{i}$, the vector $r_{i}$ points into the orthant $\mathbb{R}_{+}^{K}$. This condition is without loss of generality since it is clearly a necessary condition for the existence of a constrained version $\phi$ that stays in the orthant. The rigorous definition of the ORP is as follows. Recall the definitions of $\mathcal{D}_{\text {lim }}^{+}$and $\mathcal{I}_{0}^{+}$given in Section 1.2. 
Definition 1.1 (Oblique Reflection Problem) Given $R \in \mathbb{R}^{K \times K}$ with $R_{i i}>0$ for $i=$ $1, \ldots, K$ and $\psi \in \mathcal{D}_{\lim }^{+},(\phi, \theta) \in \mathcal{D}_{\lim }^{+} \times \mathcal{I}_{0}^{+}$solve the oblique reflection problem associated with the constraint matrix $R$ for $\psi$ if $\phi(0)=\psi(0)$, and if for all $t \in[0, \infty)$,

1. $\phi(t) \in \mathbb{R}_{+}^{K}$

2. $\phi(t)=\psi(t)+R \theta(t)$, where for every $i=1, \ldots, K$

$$
\int_{(0, t]} 1_{(0, \infty)}\left(\phi^{i}(s)\right) d \theta^{i}(s)=0
$$

Note that the condition (1.8) simply states that the constraining term $\theta^{i}$ can increase at time $t$ only if $\phi^{i}(t)=0$. From the definition above it is clear that one can without loss of generality assume that $R_{i i}=1$ for $i=1, \ldots, K$. Indeed, we shall assume this normalization throughout the rest of the paper. When a unique solution to the ORP exists for every $\psi \in \mathcal{D}_{\text {lim }}^{+}$, we say the ORP is well-defined and refer to the mapping $\Gamma: \psi \rightarrow \phi$ as the reflection map (RM). We also use $\Theta: \psi \rightarrow \theta$ to denote the mapping that takes $\psi$ to the corresponding constraining term $\theta$.

In this work we focus mainly on oblique reflection problems (ORPs) associated with reflection matrices $R$ that satisfy the so-called Harrison-Reiman (H-R) condition stated below as Definition 1.2, which was first introduced in [16]. As shown in Theorem 3.1, ORPs in this class are welldefined, and in fact have Lipschitz continuous reflection mappings (with respect to the uniform topology on path space on both the domain and range).

Definition 1.2 ( $H-R$ condition) A constraint matrix $R \in \mathbb{R}^{K \times K}$ is said to satisfy the $H-R$ condition if $P \doteq I-R \geq 0$ and the spectral radius of the matrix $P$ is less than one.

Remark 1.3 If $R$ satisfies the $H-R$ condition and $P \doteq I-R$, then there exists a diagonal matrix $A$ with strictly positive diagonal elements such that each row sum of the matrix $\tilde{P} \doteq A^{-1} P A$ is strictly less than 1 (cf., [48]), Lemma 3).

Remark 1.4 The ORP was introduced in [16] to characterize functional central limits of singleclass open queueing networks (see Figure 2). Single-class open queueing networks with $K$ queues in which, on average, a fraction $q_{i j}$ of the departures from queue $i$ are sent to queue $j$, and a fraction $1-\sum_{j=1}^{K} q_{i j}$ of the departures from queue $i$ exit the network give rise to ORPs with an $\mathbb{R}^{K \times K}$ constraint matrix $R$ given by

$$
R_{i j} \doteq\left\{\begin{aligned}
-q_{j i} & \text { for } j \neq i \\
1 & \text { otherwise. }
\end{aligned}\right.
$$


We now precisely state what we mean by a directional derivative of the multi-dimensional reflection map.

Definition 1.5 Consider an ORP whose reflection map $\Gamma$ is well-defined on $\mathcal{D}_{\text {lim. }}$. Given paths $\psi \in \mathcal{D}_{\text {lim }}^{+}, \chi \in \mathcal{D}_{\text {lim }}$, define

$$
\nabla_{\chi}^{\varepsilon} \Gamma(\psi) \doteq \frac{1}{\varepsilon}[\Gamma(\psi+\varepsilon \chi)-\Gamma(\psi)] \quad \text { for } \varepsilon>0 .
$$

The derivative of $\Gamma$ along $\chi$ evaluated at $\psi$ is the pointwise limit of the sequence $\left\{\nabla_{\chi}^{\varepsilon} \Gamma(\psi)\right\}$, as $\varepsilon \downarrow 0$.

\subsection{Main Results of the Paper}

\subsubsection{Existence of the derivative}

In $[25]$ it was shown that when $\Gamma$ is the one-dimensional $\mathrm{RM}, \psi, \chi$ are continuous, and $\Gamma(\psi)(0)=$ $\psi(0)=0$, then the directional derivative has the explicit form

$$
\nabla_{\chi} \Gamma(\psi)(t)=\chi(t)+\sup _{s \in \Phi(t)}[-\chi(s)] \vee 0
$$

where

$$
\Phi(t) \doteq\{s \in[0, t]: \phi(s)=0 \text { and } \theta(s)=\theta(t)\}
$$

with $\phi=\Gamma(\psi)$ and $\theta$ defined as in (1.1) and (1.2), respectively. In reality, this was shown in [25] under the additional restrictions that $\nabla_{\chi} \Gamma(\psi)$ has only a finite number of discontinuities in any compact interval and $\phi(0)=0$. However, as shown in Theorem 3.5 (see also [50, Theorem 9.3.1]), these conditions can be relaxed. When $\psi$ and $\chi$ are the fluid and functional central limits of the netput process associated with a time-varying queue, then $\nabla_{\chi} \Gamma(\psi)$ characterizes the secondorder approximation to the time-varying queue. In this case $\phi=\Gamma(\psi)$ has an interpretation as the fluid limit of the queue and $\theta$ as the corresponding cumulative potential outflow lost (due to idleness of the server) during the period $[0, t]$. Thus $\Phi(t)$ represents the set of all times $s$ in the interval $[0, t]$ when the fluid queue was zero, but the server was fully utilized in the interval $[s, t]$. Observe that when $\phi(0)=0$, using the fact that $\Gamma(\psi)(t)=\psi(t)+\theta(t)=\psi(t)+\overline{-\psi}(t) \geq 0$ for every $t \in[0, \infty)$, it can be shown (see Lemma 3.8) that $\Phi(t)$ can be rewritten as $\Phi(t)=\Phi_{-\psi}(t)$, where for $f \in \mathcal{D}_{\text {lim }}$,

$$
\Phi_{f}(t) \doteq\{s \in[0, t]: f(s)=\bar{f}(t)\}
$$


When $\psi, \chi \in \mathcal{D}_{\text {lim }}$ are not necessarily continuous, the directional derivative can be shown to still exist (see Theorem 3.5) but, in addition to sets of the form $\Phi_{f}$, its explicit representation also involves sets of the form

$$
\begin{aligned}
\Phi_{f}^{L}(t) & \doteq\{s \in[0, t]: f(s-)=\bar{f}(t)\} \\
\tilde{\Phi}_{f}^{R}(t) & \doteq\{s \in[0, t): f(s+)=\bar{f}(t)\} .
\end{aligned}
$$

Now consider the multi-dimensional setting, when $\Gamma$ is the RM associated with an ORP with an H-R constraint matrix $R \in \mathbb{R}^{K \times K}$ and $(\phi, \theta)$ solve the ORP for a given $\psi \in \mathcal{D}_{\text {lim. }}$. In this setting, for $i=1, \ldots, K, \theta^{i}$ represents the cumulative potential outflow lost from the $i$ th queue during $[0, t]$, and the set

$$
\Phi^{i}(t) \doteq\left\{s \in[0, t]: \phi^{i}(s)=0 \text { and } \theta^{i}(s)=\theta^{i}(t)\right\}
$$

represents the times $s \in[0, t]$ at which the $i$ th fluid queue is zero but the $i$ th server is fully utilized during $[s, t]$. As shown below in Theorem 1.6, when $\psi, \chi$ are continuous, the directional derivative in the multi-dimensional case can be expressed in terms of these sets. The proof of Theorem 1.6 is given in Section 3.4. Recall, from Section 1.2 that $\Delta f(t+) \neq 0$ denotes the condition that the function $f$ is not flat to the right of $t$, and also recall the convention that $\inf \emptyset=-\infty$.

Theorem 1.6 (Existence and Characterization of $\nabla_{\chi} \Gamma(\psi)$ ) Let $R \in \mathbb{R}^{K \times K}$ be a reflection matrix that satisfies the $H-R$ condition stated in Definition 1.2, let $P \doteq I-R$ and let $\Gamma$ be the associated RM. Then the following properties hold.

1. Given $\psi, \chi \in \mathcal{D}_{\text {lim }}$, the directional derivative $\nabla_{\chi} \Gamma(\psi)$ exists and lies in $\mathcal{D}_{\text {lim }}$. Furthermore, the derivative $\nabla_{\chi} \Gamma(\psi)$ is Lipschitz in $\chi$ (with respect to the uniform topology on both the domain and range) and, for every $\alpha, \beta>0$, also satisfies

$$
\nabla_{\alpha \chi} \Gamma(\beta \psi)=\alpha \nabla_{\chi} \Gamma(\psi)
$$

2. When $\psi, \chi \in \mathcal{C}$, the convergence of $\nabla_{\chi}^{\varepsilon} \Gamma(\psi)$ to $\nabla_{\chi} \Gamma(\psi)$ is uniform on compact subsets of continuity points of $\nabla_{\chi} \Gamma(\psi)$. Moreover, if $(\phi, \theta)$ solve the ORP for $\psi$, then

$$
\nabla_{\chi} \Gamma(\psi)=\chi+R \gamma_{(1)}(\psi, \chi)
$$


where $\gamma_{(1)} \doteq \gamma_{(1)}(\psi, \chi)$ lies in $\mathcal{D}_{\text {usc }}$ and is the unique solution to the system of equations

$$
\gamma^{i}(t)= \begin{cases}0 & \text { if } t \in\left(0, t_{l}^{i}\right) \\ \sup _{s \in \Phi^{i}(t)}\left[-\chi^{i}(s)+[P \gamma]^{i}(s)\right] \vee 0 & \text { if } t \in\left[t_{l}^{i}, t_{u}^{i}\right] \\ \sup _{s \in \Phi^{i}(t)}\left[-\chi^{i}(s)+[P \gamma]^{i}(s)\right] & \text { if } t \in\left(t_{u}^{i}, \infty\right)\end{cases}
$$

for $i=1, \ldots, K$, with $\Phi^{i}$ defined as in (1.14) and

$$
\begin{aligned}
t_{l}^{i} & =\inf \left\{t>0: \phi^{i}(t)=0\right\} \\
t_{u}^{i} & =\inf \left\{t>0: \Delta \theta^{i}(t+) \neq 0\right\} .
\end{aligned}
$$

3. When $\psi \in \mathcal{D}_{c}$ and $\chi \in \mathcal{D}_{\lim }$, if $(\phi, \theta)$ solve the ORP for $\psi$, then

$$
\nabla_{\chi} \Gamma(\psi)=\chi+R \gamma_{(2)}(\psi, \chi)
$$

where $\gamma_{(2)}=\gamma_{(2)}(\psi, \chi)$ is the unique solution to the system of equations

$$
\gamma^{i}(t)= \begin{cases}0 & \text { if } t \in\left(0, t_{l}^{i}\right) \\ S_{2}\left(-\psi^{i}+[P \theta]^{i},-\chi^{i}+[P \gamma]^{i}\right) \vee 0 & \text { if } t \in\left[t_{l}^{i}, t_{u}^{i}\right] \\ S_{2}\left(-\psi^{i}+[P \theta]^{i},-\chi^{i}+[P \gamma]^{i}\right) & \text { if } t \in\left(t_{u}^{i}, \infty\right)\end{cases}
$$

for $i=1, \ldots, K$, where $t_{l}^{i}$ and $t_{u}^{i}$ are defined by (1.18) and (1.19), respectively, and for $f, g \in \mathcal{D}_{\lim }$,

$$
S_{2}(f, g)=\sup _{s \in \Phi_{f}^{L}(t)}[g(s-)] \vee \sup _{s \in \Phi_{f}(t)}[g(s)] \vee \sup _{s \in \tilde{\Phi}_{f}^{R}(t)}[g(s+)]
$$

Remark 1.7 Note that if $\psi, \chi$ are continuous, $\phi(0)=0$ and $\Delta \theta^{i}(t+)=0$ for every $t \in[0, \infty)$. This corresponds to the case when all fluid queues are initially empty and are subsequently always in heavy-traffic or, equivalently, are always critically loaded. In this case, $t_{l}^{i}=0, t_{u}^{i}=\infty$ (due to the convention that the supremum of an empty set is $\infty$ ) and $\Phi^{i}(t)=[0, t]$ for every $i=1, \ldots, K$ and $t \in[0, \infty)$. In this case $\gamma_{(1)}$ is the unique solution to the system of equations

$$
\gamma^{i}(t)=\sup _{s \in[0, t]}\left[-\chi^{i}(s)+[P \gamma]^{i}(s)\right] \vee 0 \quad \text { for } i=1, \ldots, K .
$$

It then follows from Theorem 3.1 (see also equations (13)-(15) and Theorem 1 of [16]) that the derivative is simply the reflected or constrained version of $\chi$ :

$$
\nabla_{\chi} \Gamma(\psi)=\chi+R \gamma_{(1)}=\Gamma(\chi)
$$

which is consistent with the well-known reflected Brownian motion characterization of heavytraffic limits of stationary open single-class queueing networks [16, 41]. 


\subsubsection{Discontinuities of the derivative $\nabla_{\chi} \Gamma(\psi)$ for continuous $\psi, \chi$}

Theorem 1.6 shows that even when $\psi, \chi \in \mathcal{C}$, convergence of $\nabla_{\chi}^{\varepsilon} \Gamma(\psi)$ to $\nabla_{\chi} \Gamma(\psi)$ is pointwise and is uniform only on compact subsets of continuity points of the derivative $\nabla_{\chi} \Gamma(\psi)$. In order to establish convergence with respect to stronger topologies than the pointwise topology (which is carried out in [39]), it is therefore necessary to understand the structure of the discontinuities of $\nabla_{\chi} \Gamma(\psi)$. The next main result of the paper, Theorem 1.10, describes the various types of discontinuities exhibited by the derivative. It turns out that discontinuities in $\nabla_{\chi} \Gamma(\psi)$ can occur only at points at which there is a change in certain regimes associated with the solution $(\phi, \theta)$ to the ORP with input $\psi$. These regimes, which are introduced in Definition 1.8, are described in terms of the following set-valued functions. For $t \in[0, \infty)$, define

$$
\begin{aligned}
\mathcal{O}(t) & \doteq\left\{i \in\{1, \ldots, K\}: \phi^{i}(t)>0\right\} \\
\mathcal{U}(t) & \doteq\left\{i \in\{1, \ldots, K\}: \phi^{i}(t)=0, \Delta \theta^{i}(t+) \neq 0, \Delta \theta^{i}(t-) \neq 0\right\}, \\
\mathcal{C}(t) & \doteq\{1, \ldots, K\} \backslash[\mathcal{O}(t) \cup \mathcal{U}(t)] \\
\mathcal{E} \mathcal{O}(t) & \doteq\left\{i \in \mathcal{C}(t): \exists \delta>0 \text { such that } \phi^{i}(s)>0 \quad \forall s \in(t-\delta, t)\right\} \\
\mathcal{S U}(t) & \doteq\left\{i \in \mathcal{C}(t): \Delta \theta^{i}(t-)=0, \Delta \theta^{i}(t+) \neq 0\right\} .
\end{aligned}
$$

When $\psi$ is the fluid limit of the so-called netput process associated with a queueing network, then $\mathcal{O}(t)$ represents the set of queues that are overloaded at time $t, \mathcal{U}(t)$ the set of queues that are underloaded at time $t$ and $\mathcal{C}(t)$ the set of queues that are critical at time $t$. Moreover, $\mathcal{S U}(t)$ represents the set of queues that are at the start of underloading and $\mathcal{E O}(t)$ the set of queues that are at the end of overloading at time $t$. The terminology used in the following definition reflects this interpretation of the various regimes of $(\phi, \theta)$.

Definition 1.8 (Regimes of $(\phi, \theta)$ ) Given an ORP associated with an $H$-R reflection matrix, let $(\phi, \theta)$ be the solution to the ORP for a given input trajectory $\psi$. Then $i \in \mathcal{I}$ is said to be overloaded (respectively critical, underloaded, at the start of underloading, at the end of overloading) at time $t$ if and only if $i \in \mathcal{O}(t)$ (respectively $i \in \mathcal{C}(t), i \in \mathcal{U}(t), i \in \mathcal{S U}(t), i \in \mathcal{E} \mathcal{O}(t)$ ).

In [25], strong approximations for the uniformly accelerated $M_{t} / M_{t} / 1$ queue with integrable average instantaneous arrival and service rates $\lambda(\cdot)$ and $\mu(\cdot)$ were obtained. The second-order term in the expansion for the queue length process obtained in [25] admits an interpretation as the directional derivative $\nabla_{\chi} \Gamma(\psi)$ of the one-dimensional $\operatorname{RM} \Gamma$, where $\psi \in \mathcal{C}$ is equal to the 
fluid netput process given by

$$
\psi(t)=\int_{0}^{t} \lambda(s) d s-\int_{0}^{t} \mu(s) d s
$$

In [25], the queue $\phi=\Gamma(\psi)$ was said to be overloaded, critical or underloaded depending on whether the traffic intensity function

$$
\rho^{*}(t) \doteq \sup _{0 \leq s \leq t} \frac{\int_{s}^{t} \lambda(r) d r}{\int_{s}^{t} \mu(r) d r}
$$

is greater than, equal to or less than 1, respectively. The regimes introduced here in Definition 1.8, while coinciding with the definition given in [25] for the one-dimensional case when $\psi$ has the particular form (1.24) (this can be seen by comparing Proposition 7.2 of [25] with Lemma 4.3 of this paper), allows for more general $\psi \in \mathcal{C}$ that are not necessarily even of bounded variation and also generalizes to the multi-dimensional setting. For the one-dimensional $\mathrm{RM} \Gamma, \psi$ of the form (1.24) and $\chi \in \mathcal{C}$, under the additional assumption that the derivative has only a finite number of discontinuities in a bounded interval, it was shown in [25] that the derivative $\nabla_{\chi} \Gamma(\psi)$ is either right or left continuous at every point. In the multi-dimensional setting, the situation is considerably more complex with components of $\nabla_{\chi} \Gamma(\psi)$ even admitting points of separated discontinuities (see (LRc) of Theorem 1.10). The following concept of critical and sub-critical chains captures the relevant aspects of the reflection matrix $R$ (or, equivalently, of the topology of the associated network) that influence the nature of discontinuities of the derivative $\nabla_{\chi} \Gamma(\psi)$.

Definition 1.9 (Critical and Sub-critical Chains) Given an $H-R$ constraint matrix $R \in$ $\mathbb{R}^{K \times K}, P \doteq I-R$, associated $R M \Gamma$ and $\psi \in \mathcal{C}$, let $\phi \doteq \Gamma(\psi)$. Then a sequence $j_{0}, j_{1}, j_{2}, \ldots, j_{m}$, with $j_{k} \in\{1, \ldots, K\}$ for $k=0,1, \ldots, m$, that satisfies $P_{j_{k-1} j_{k}}>0$ for $k=1, \ldots, m$ is said to be a chain. The chain is said to be a cycle if there exist distinct $k_{1}, k_{2} \in\{0, \ldots, m\}$ such that $j_{k_{1}}=j_{k_{2}}$, is said to precede $i$ if $j_{0}=i$ and is said to be empty at $t$ if $\phi^{j_{k}}(t)=0$ for every $k=1, \ldots, m$. For $i=1, \ldots, K$ and $t \in[0, \infty)$, we consider the following two types of chains.

1. At $t$, an empty chain preceding $i$ is said to be critical if it is either cyclic or $m$ is at the end of overloading at $t$.

2. At $t$, an empty chain preceding $i$ is said to be sub-critical if it is either cyclic or $m$ is at the start of underloading at $t$.

We now state the second main result of the paper, which specifies necessary conditions for the existence of left and right discontinuities of $\nabla_{\chi} \Gamma(\psi)$ when $\psi, \chi$ are continuous. 
Theorem 1.10 (Necessary Conditions for Discontinuities in $\nabla_{\chi} \Gamma(\psi)$ ) Given an H-R constraint matrix $R$ with associated reflection map $\Gamma$ and functions $\psi, \chi \in \mathcal{C}$, the directional derivative $\nabla \Gamma \doteq \nabla_{\chi} \Gamma(\psi)$ satisfies the following properties hold at each $t \in[0, \infty)$.

(L) If $\nabla \Gamma^{i}$ has a left discontinuity, then one of the following conditions must hold:

(a) $i$ is at the end of overloading and no critical chain precedes $i$, in which case

$$
\nabla \Gamma^{i}(t-)<\nabla \Gamma^{i}(t)=0
$$

(b) $i$ is not underloaded and a critical chain precedes $i$; if, in addition, $i$ is overloaded then

$$
\nabla \Gamma^{i}(t-)>\nabla \Gamma^{i}(t)
$$

(R) If $\nabla \Gamma^{i}$ has a right discontinuity, then one of the following conditions must hold:

(a) $i$ is at the start of underloading and no sub-critical chain precedes $i$, in which case

$$
\nabla \Gamma^{i}(t)>\nabla \Gamma^{i}(t+)=0
$$

(b) $i$ is not underloaded and a sub-critical chain precedes $i$; if $i$ is also overloaded then

$$
\nabla \Gamma^{i}(t)<\nabla \Gamma^{i}(t+)
$$

(LR) If $\nabla \Gamma^{i}$ has both a right and left discontinuity, then one of the following conditions holds: (a) $i$ is at the end of overloading, no critical chain precedes $i$ but a sub-critical chain precedes $i$, in which case

$$
\nabla \Gamma^{i}(t-)<\nabla \Gamma^{i}(t)=0<\nabla \Gamma^{i}(t+)
$$

(b) $i$ is at the start of underloading, no sub-critical chain precedes $i$ but a critical chain precedes $i$, in which case

$$
0=\nabla \Gamma^{i}(t-)>\nabla \Gamma^{i}(t)>\nabla \Gamma^{i}(t+)
$$

(c) $i$ is not underloaded and there exist both critical and sub-critical chains preceding $i$; if, in addition, $i$ is overloaded then the discontinuity is a separated discontinuity of the form

$$
\nabla \Gamma^{i}(t)<\min \left[\nabla \Gamma^{i}(t-), \nabla \Gamma^{i}(t+)\right]
$$

Finally, if $i$ is underloaded at $t$ then $\nabla \Gamma^{i}(t-)=\nabla \Gamma^{i}(t)=\nabla \Gamma^{i}(t+)=0$. 


\section{Connection with Queueing Networks}

In Section 2.1 we provide a heuristic description of how directional derivatives of multi-dimensional reflection maps arise in the characterization of second-order (or functional central limit) approximations to non-stationary queueing networks. In Section 2.2 we present two examples to illustrate how the topology of a queueing network associated with a reflection matrix $R$ and the various states of the fluid $(\phi, \theta)$ associated with a continuous netput process $\psi$ can influence the nature of discontinuities of the associated directional derivative $\nabla_{\chi} \Gamma(\psi)$.

\subsection{Directional Derivatives and Functional Central Limits}

Second-order or diffusion approximations of many classes of queueing networks can be obtained by the following general procedure $[7,8,27,40,41,49]$. Consider a family of queueing networks defined in terms of their primitives (i.e. the random processes defined on some probability space $(\Omega, \mathcal{F}, P)$ that describe arrivals, services and routing, as well as the scheduling rules). For each queueing network in the family, one constructs from the primitives a certain netput process, $\tilde{X}^{\varepsilon}$, where, roughly speaking, the $i$ th component of $\tilde{X}^{\varepsilon}$ represents the cumulative net arrivals minus the potential services at the $i$ th queue (see, for example, $[7,8,27,40,41,49]$ for precise definitions of netput processes associated with various queueing networks). The evolution of the corresponding queue length process, $\tilde{Z}^{\varepsilon}$, coincides with the evolution of the netput process $\tilde{X}^{\varepsilon}$ whenever all queues are non-empty, but in general the queue length process is a more complicated functional of the netput process: $\tilde{Z}^{\varepsilon}=\Gamma\left(\tilde{Z}^{\varepsilon}(0)+\tilde{X}^{\varepsilon}\right)$, where the functional $\Gamma$ is the multidimensional oblique reflection mapping associated with the queueing network. In many cases, the family of netput processes $\left\{\tilde{X}^{\varepsilon}\right\}$ can be assumed to be Markovian and satisfy a functional strong law of large numbers (FSLLN) and functional central limit theorem (FCLT). Specifically, for $\varepsilon>0$, consider the so-called uniformly accelerated version $\bar{X}^{\varepsilon}$ of $\tilde{X}^{\varepsilon}$, where $\bar{X}^{\varepsilon} / \varepsilon^{2}$ is defined to be the Markovian process whose instantaneous transition rates are equal to the instantaneous transition rates of $\tilde{X}^{\varepsilon}$ scaled by $1 / \varepsilon^{2}$. (See $[19,34,31,32]$ for more discussion on the uniform acceleration scaling applied to queueing networks.) Note that in the time-homogeneous setting, $\bar{X}^{\varepsilon}$ can be equivalently defined by

$$
\bar{X}^{\varepsilon}(t) \doteq \varepsilon^{2} \tilde{X}^{\varepsilon}\left(t / \varepsilon^{2}\right) \quad \text { for } t \in[0, \infty) .
$$


The FSLLN for the family of netput processes then takes the form

$$
\bar{X}^{\varepsilon} \rightarrow \bar{X} \quad \text { as } \varepsilon \rightarrow 0,
$$

where the limit is in the sense of $P$-a.s. convergence with respect to an appropriate topology on path space (e.g. uniform convergence on compact sets). Similarly, the FCLT for the netput process takes the form

$$
\hat{X}^{\varepsilon} \Rightarrow \hat{X} \quad \text { as } \varepsilon \rightarrow 0
$$

where the limit is in the sense of weak convergence and

$$
\hat{X}^{\varepsilon} \doteq \frac{1}{\varepsilon}\left[\bar{X}^{\varepsilon}-\bar{X}\right]
$$

is a rescaled centered version of the netput process that captures the fluctuations around its FSSLN limit.

In order to obtain a corresponding FSLLN and FCLT for the queue length process, in analogy with $\bar{X}^{\varepsilon}, \bar{Z}^{\varepsilon}$ is first defined to be the corresponding uniformly accelerated version of $\tilde{Z}^{\varepsilon}$. Next, homogeneity of the reflection map $\Gamma$ with respect to space and time can be used to conclude that

$$
\bar{Z}^{\varepsilon}=\Gamma\left(\bar{Z}^{\varepsilon}(0)+\bar{X}^{\varepsilon}\right)
$$

Under the assumption that $\bar{Z}^{\varepsilon}(0) \rightarrow \bar{Z}(0)$ as $\varepsilon \rightarrow 0$, the FSLLN for the queue length process is then obtained by establishing the $P$-a.s. convergence

$$
\bar{Z}^{\varepsilon}=\Gamma\left(\bar{Z}^{\varepsilon}(0)+\bar{X}^{\varepsilon}\right) \rightarrow \bar{Z} \doteq \Gamma(\bar{Z}(0)+\bar{X}) \quad \text { as } \varepsilon \rightarrow 0,
$$

where $\bar{X}$ is the FSLLN limit of the netput process. The process $\bar{Z}$ provides a first-order approximation to the queueing network and is referred to as the fluid limit of the queueing network. To capture the fluctuations of the queue lengths around the fluid limit, one then considers the centered sequence $\left\{\hat{Z}^{\varepsilon}\right\}$ of queue lengths defined by

$$
\hat{Z}^{\varepsilon} \doteq \frac{1}{\varepsilon}\left[\bar{Z}^{\varepsilon}-\bar{Z}\right] \quad \text { for } \varepsilon>0 \text {. }
$$

The above display, together with (2.2), (2.3) and (2.4), then yields the relation

$$
\hat{Z}^{\varepsilon}=\frac{1}{\varepsilon}\left[\Gamma\left(\bar{Z}^{\varepsilon}(0)+\bar{X}^{\varepsilon}\right)-\Gamma(\bar{Z}(0)+\bar{X})\right]=\frac{1}{\varepsilon}\left[\Gamma\left(\bar{Z}^{\varepsilon}(0)+\bar{X}+\varepsilon \hat{X}^{\varepsilon}\right)-\Gamma(\bar{Z}(0)+\bar{X})\right] .
$$


In many cases, using continuity properties of $\Gamma$ and the FCLT (2.1), it is then possible to show that (with respect to a suitable topology on path space) the limit $\hat{Z} \doteq \lim _{\varepsilon \rightarrow 0} \hat{Z}^{\varepsilon}$ exists and satisfies

$$
\hat{Z}=\lim _{\varepsilon \rightarrow 0} \frac{1}{\varepsilon}[\Gamma(\bar{Z}(0)+\bar{X}+\varepsilon \hat{X})-\Gamma(\bar{Z}(0)+\bar{X})]=\nabla_{\hat{X}} \Gamma(\bar{Z}(0)+\bar{X}),
$$

where $\nabla_{\hat{X}} \Gamma(\bar{X})$ is the directional derivative of the reflection map $\Gamma$ (see Definition 1.5) in the direction $\hat{X}$, evaluated at $\bar{Z}(0)+\bar{X}$. In summary, under appropriate conditions, the fluid limit or first-order approximation, $\bar{Z}$, and the functional central limit or second-order approximation, $\hat{Z}$, of the queue length process have the representations

$$
\bar{Z}=\Gamma(\bar{Z}(0)+\bar{X}) \quad \text { and } \quad \hat{Z}=\nabla_{\hat{X}} \Gamma(\bar{Z}(0)+\bar{X})
$$

where $\bar{X}$ and $\hat{X}$ are the functional strong law and functional central limits, respectively, of the netput process. As explained in Remark 1.7, for time-homogeneous networks, under socalled heavy traffic conditions, the representations for fluid and functional central limits for the queueing network take the simpler, more familiar form $\bar{Z} \equiv 0$ and $\hat{Z}=\Gamma(\hat{X})$. On the other hand, in order to analyze time-inhomogeneous networks or transient behaviour in time-homogeneous networks (i.e. when $\bar{Z}(0) \neq 0$ ), the fluid limit is in general not trivial, and so the second-order approximation is no longer equal to the image of $\hat{X}$ under the reflection map, but instead involves a certain directional derivative of the oblique reflection map.

\section{$2.2 \quad$ Illustrative Examples}

Here we show how directional derivatives associated with two time-inhomogeneous networks a two-station tandem queueing network in Section 2.2.1 and a three-station "join" network in Section 2.2.2 - can be computed. In both examples, $\lambda^{i}$ denotes the mean exogenous arrival rate to station $i$ and $\mu^{i}$ the mean potential service rate at station $i$. Moreover, the netput process, $\psi$, which represents the cumulative net arrivals minus the cumulative potential services that the queues would have seen had they been non-empty throughout, is defined by the equations $\psi^{i}(t)=\int_{0}^{t}\left(\lambda^{i}(s)+[P \mu(s)]^{i}-\mu^{i}(s)\right) d s$ for $t \in[0, T]$ and $i=1, \ldots, K$. 


\subsubsection{A tandem queueing model}

Consider the two-station tandem queueing system illustrated in Figure 2. The associated reflection map has routing matrix $Q$ and reflection matrix $R=I-Q^{T}$ given by

$$
Q \doteq\left[\begin{array}{cc}
0 & 1 \\
0 & 0
\end{array}\right] \quad \text { and } \quad R \doteq\left[\begin{array}{cc}
1 & 0 \\
-1 & 1
\end{array}\right]
$$

Since $Q^{T} \geq 0$ and has spectral radius zero, $R$ satisfies the Harrison-Reiman condition stated in Definition 1.2. Let $\Gamma$ denote the associated reflection map (see Figure 1 for the geometry of the associated ORP).

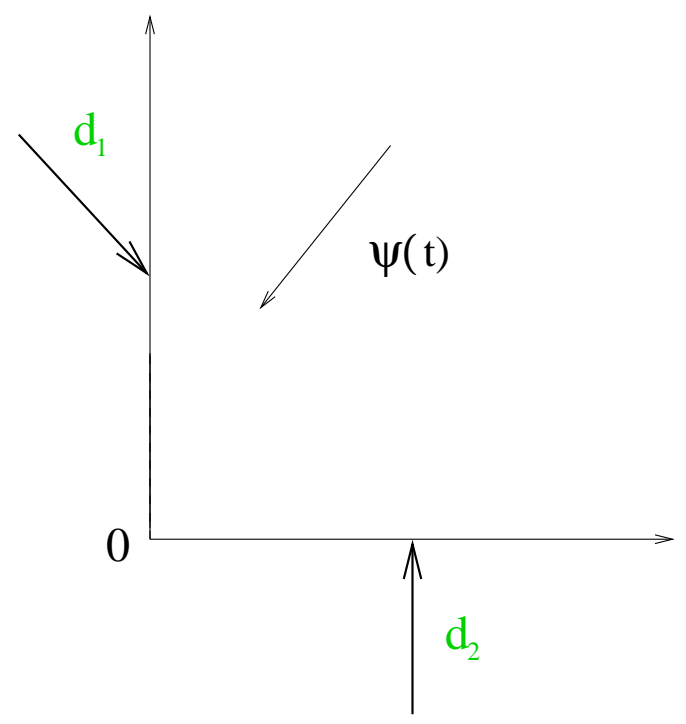

Figure 1: The oblique reflection problem (ORP) associated with the tandem queueing network.

We consider a model in which there are no exogeneous arrivals to station 2, arrivals to station 1 occur at a time-dependent mean rate of $\lambda^{1}(\cdot)$ given by

$$
\lambda^{1}(t) \doteq\left\{\begin{array}{cc}
3 & \text { for } t \in[0,1) \\
1 & \text { for } t \in[1,3]
\end{array}\right.
$$

and the mean potential service rates at station 1 and station 2 are constant and given by $\mu^{1}=2$ and $\mu^{2}=1$, respectively. If $\psi$ is the netput process and $(\phi, \theta)$ solve the ORP for $\psi$ then, as 
shown in Figure 2, it it is easy to see that $\psi^{2}(t)=t$ and $\theta^{2}(t)=0$ for $t \in[0,3]$ and

$$
\begin{aligned}
& \psi^{1}(t)=\left\{\begin{array}{rl}
t & \text { for } t \in[0,1) \\
1-(t-1) & \text { for } t \in[1,3],
\end{array} \quad \phi^{1}(t)=\left\{\begin{array}{rl}
t & \text { for } t \in[0,1) \\
1-(t-1) & \text { for } t \in[1,2) \\
0 & \text { for } t \in[0,2) \\
\theta^{1}(t)=\left\{\begin{aligned}
& 0 \text { for } t \in[2,3], \\
&(t-2) \text { for } t \in[2,3],
\end{aligned}\right.
\end{array} \quad \phi^{2}(t)=\left\{\begin{aligned}
t & \text { for } t \in[0,2) \\
2 & \text { for } t \in[2,3],
\end{aligned}\right.\right.\right.
\end{aligned}
$$
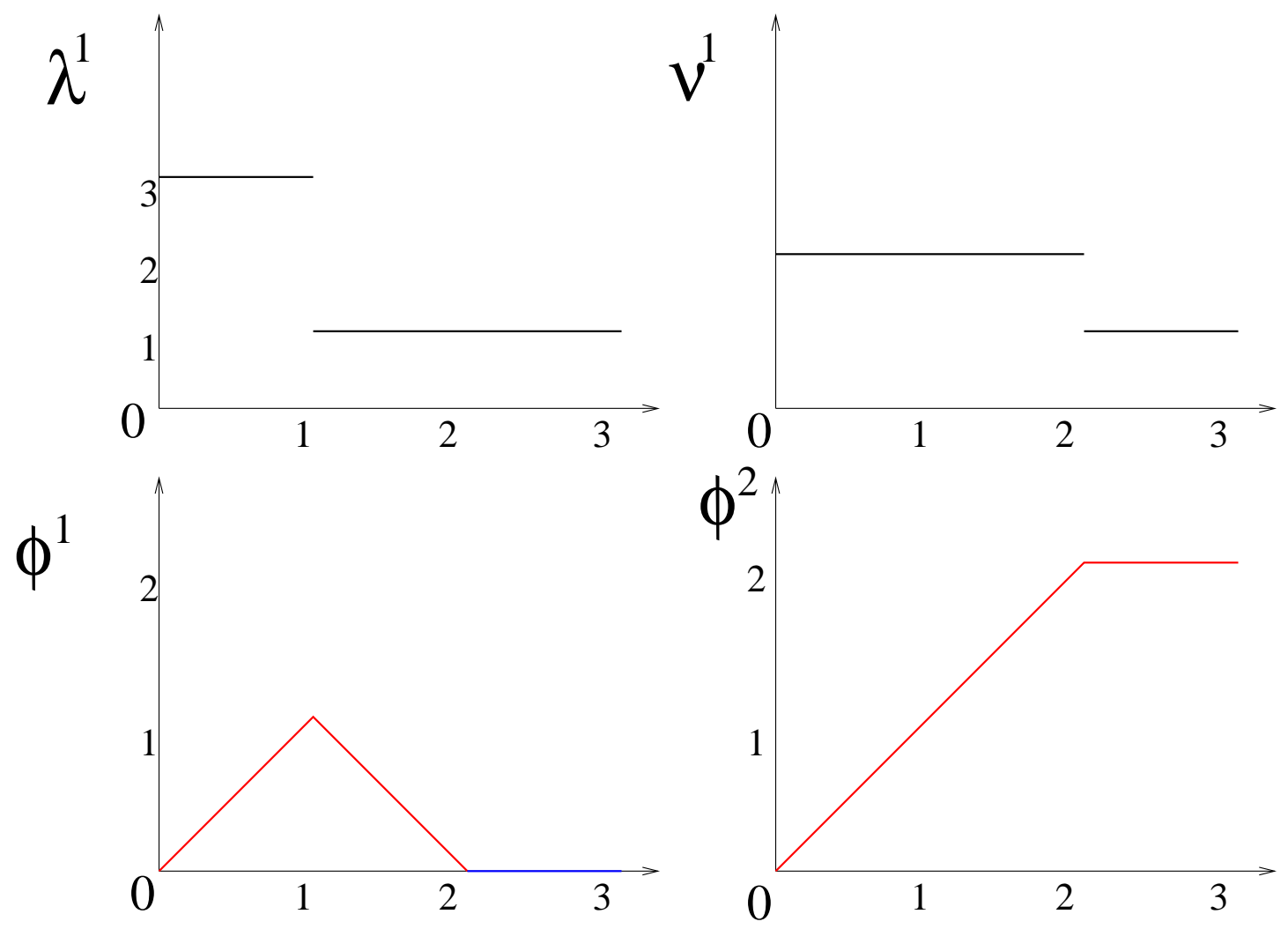

Figure 2: The time-varying exogenous arrival rate $\lambda^{1}$ to and departure rate $\nu^{1}$ from the first queue, along with the contents $\phi^{1}$ and $\phi^{2}$ of the first and second queue in the tandem network.

The above also relations show that $t_{l}^{1}=t_{l}^{2}=0, t_{u}^{1}=2, t_{u}^{2}=\infty$ and, by the representation (1.14) for $\Phi^{i}$, we see that $\Phi^{2}(t)=\{0\}$ for $t \in[0, \infty)$ and

$$
\Phi^{1}(t) \doteq\left\{\begin{aligned}
\{0\} & \text { for } t \in[0,2) \\
\{0,2\} & \text { for } t=2 \\
\{t\} & \text { for } t \in(2,3]
\end{aligned}\right.
$$


Now fix $\chi \in \mathcal{C}$. Since $\psi \in \mathcal{C}$, we can use the characterization (1.17) and the formulae for $\Phi^{i}$ given above to compute the directional derivative $\nabla \Gamma=\nabla_{\chi} \Gamma(\psi)$ as follows: $\nabla \Gamma^{1}(t)=\chi^{1}(t)+\gamma^{1}(t)$, where

$$
\gamma^{1}(t)=\left\{\begin{aligned}
{\left[-\chi^{1}(0)\right] \vee 0 } & \text { for } t \in[0,2) \\
{\left[-\chi^{1}(2)\right] \vee\left[-\chi^{1}(0)\right] \vee 0 } & \text { for } t=2 \\
-\chi^{1}(t) & \text { for } t \in(2,3]
\end{aligned}\right.
$$

while $\nabla \Gamma^{2}(t)=\chi^{2}(t)+\gamma^{2}(t)-\gamma^{1}(t)=\chi^{2}(t)+\gamma^{2}(0)-\gamma^{1}(t)$, where

$$
\gamma^{2}(0)=-\chi^{2}(0)+\gamma^{1}(0)=\chi^{2}(0)+\left[-\chi^{1}(0)\right] \vee 0
$$

We now refer to the various types of discontinuities mentioned in Theorem 1.10. From the above expressions, it is clear that at $t=2,\left[-\chi^{1}(2)\right]>\left[-\chi^{1}(0)\right] \vee 0$ is a necessary and sufficient condition for $\nabla \Gamma^{1}$ to have a left discontinuity (of type (La)) as well as for $\nabla \Gamma^{2}$ to have a left discontinuity (of type $(\mathrm{Lb})$ ), while the reverse inequality, $\left[-\chi^{1}(2)\right]<\left[-\chi^{1}(0)\right] \vee 0$, is necessary and sufficient for $\nabla \Gamma^{1}$ to have a right discontinuity (of type (Ra)) as well as for $\nabla \Gamma^{2}$ to have a right discontinuity (of type $(\mathrm{Rb})$ ). Observe that the necessary conditions mentioned in Theorem 1.10 are indeed satisfied since at $t=2$, queue 1 is at the end of overloading and at the start of underloading, while queue 2 is overloaded and has critical and sub-critical chains preceding it.

\subsubsection{A merge or join network}

We now consider a scenario in which two upstream queues feed into a common buffer (see Figure 3). The upstream queues experience a surge in arrival rate for an initial period, which then subsides to a lower rate. However, just as the surge ends, the server at queue 2 undergoes a partial failure, resulting in the queue maintaining criticality. We show that in such a scenario there can be a discontinuity in the derivative of the downstream queue at the time congestion ends in the upstream queues.

There are no exogeneous arrival rates to queue 3 and the mean exogenous arrival rate $\lambda^{i}$ to queue $i$ for $i=1,2$ is given by

$$
\lambda^{1}(t)=\left\{\begin{array}{ll}
1 & \text { for } t \in[0,1) \\
1 / 2 & \text { for } t \in[1,2]
\end{array} \quad \text { and } \quad \lambda^{2}(t)= \begin{cases}3 / 2 & \text { for } t \in[0,1 / 2) \\
1 / 2 & \text { for } t \in[1 / 2,1) \\
1 / 3 & \text { for } t \in[1,2]\end{cases}\right.
$$


Moreover, we assume that queues 1 and 3 have constant service rates $\mu^{1}(t)=\mu^{3}(t)=1$ for $t \in[0,2]$, while queue 2 has service rate

$$
\mu^{2}(t)= \begin{cases}1 & \text { for } t \in[0,1] \\ 1 / 3 & \text { for } t \in[1,2]\end{cases}
$$

Since the departures from queues 1 and 2 feed into queue 3 (see Figure 3), the routing matrix $Q$ and reflection matrix $R=I-Q^{T}$ are given by

$$
Q=\left[\begin{array}{ccc}
0 & 0 & 0 \\
0 & 0 & 0 \\
1 & 1 & 0
\end{array}\right] \quad \text { and } \quad R=I-Q^{T}=\left[\begin{array}{ccc}
1 & 0 & -1 \\
0 & 1 & -1 \\
0 & 0 & 1
\end{array}\right]
$$

It is trivial to verify that $Q^{T}$ is an $\mathrm{H}-\mathrm{R}$ matrix. Let $\Gamma$ denote the associated reflection map, $\psi$ the netput process and let $\phi=\Gamma(\psi)$. Then it follows from the definitions that

$$
\psi^{1}(t)=\left\{\begin{array}{rl}
0 & \text { for } t \in[0,1) \\
-\frac{1}{2}(t-1) & \text { for } t \in[1,2],
\end{array} \quad \theta^{1}(t)=\left\{\begin{aligned}
0 & \text { for } t \in[0,1) \\
\frac{1}{2}(t-1) & \text { for } t \in[1,2],
\end{aligned}\right.\right.
$$

$\phi^{1}, \theta^{2}$ and $\theta^{3}$ are identically zero on $[0,2]$, so that $\phi^{2}=\psi^{2}$ and $\phi^{3}=\psi^{3}$ are given by

$$
\phi^{2}(t)=\left\{\begin{array}{rl}
\frac{1}{2} t & \text { for } t \in[0,1 / 2) \\
\frac{1}{4}-\frac{1}{2}(t-1 / 2) & \text { for } t \in[1 / 2,1) \\
0 & \text { for } t \in[1,2],
\end{array} \quad \phi^{3}(t)= \begin{cases}t & \text { for } t \in[0,1) \\
1-\frac{1}{6}(t-1) & \text { for } t \in[1,2] .\end{cases}\right.
$$

Figure 4 provides an illustration of the fluid limit $\phi$ of the three queues.

The above calculations also readily show that $\Phi^{3}(t)=\{0\}$ for $t \in[0,2]$,

$$
\Phi^{1}(t)=\left\{\begin{array}{ll}
{[0, t]} & \text { for } t \in[0,1] \\
\{t\} & \text { for } t \in(1,2]
\end{array} \quad \text { and } \quad \Phi^{2}(t)= \begin{cases}\{0\} & \text { for } t \in[0,1) \\
\{0\} \cup[1, t] & \text { for } t \in[1,2] .\end{cases}\right.
$$

From Theorem 1.6(2) it then follows that for $\chi \in \mathcal{C}, \nabla \Gamma=\nabla_{\chi} \Gamma(\psi)$ takes the explicit form

$$
\nabla \Gamma^{1}(t)=\chi^{1}(t)+\sup _{s \in \Phi^{1}(t)}\left[-\chi^{1}(s)\right], \quad \nabla \Gamma^{2}(t)=\chi^{2}(t)+\sup _{s \in \Phi^{2}(t)}\left[-\chi^{2}(s)\right]
$$

and

$$
\begin{aligned}
\nabla \Gamma^{3}(t)= & \chi^{3}(t)-\sup _{s \in \Phi^{2}(t)}\left[-\chi^{2}(s)\right]-\sup _{s \in \Phi^{1}(t)}\left[-\chi^{1}(s)\right] \\
& +\sup _{s \in \Phi^{3}(t)}\left[-\chi^{3}(s)+\sup _{r \in \Phi^{2}(s)}\left[-\chi^{2}(r)\right]+\sup _{r \in \Phi^{1}(s)}\left[-\chi^{1}(r)\right]\right]
\end{aligned}
$$


From the above expressions it is straightforward to deduce that

$$
\nabla \Gamma^{3}(1)-\nabla \Gamma^{3}(1-)=\left[\chi^{2}(1)-\chi^{2}(0)\right] \wedge 0 \quad \text { and } \quad \nabla \Gamma^{3}(1)-\nabla \Gamma^{3}(1+)=-\chi^{1}(1)-\sup _{s \in[0,1]}\left[-\chi^{1}(t)\right]
$$

Therefore, if $\chi^{2}(1)<\chi^{2}(0)$ and $\sup _{s \in[0,1]}\left[-\chi^{1}(s)\right]>-\chi^{1}(1)$, then

$$
\nabla \Gamma^{3}(1)-\nabla \Gamma^{3}(1-)=\chi^{2}(1)-\chi^{2}(0)<0 \quad \text { and } \quad \nabla \Gamma^{3}(1)-\nabla \Gamma^{3}(1+)=-\sup _{s \in[0,1]}\left[-\chi^{1}(s)\right]-\chi^{1}(1)<0
$$

which implies $\nabla \Gamma^{3}$ is neither right or left continuous at $t=1$. In fact, it has a separated discontinuity at that point since $\nabla \Gamma^{3}(1)<\nabla \Gamma^{3}(1-) \wedge \nabla \Gamma^{3}(1+)$, as anticipated by condition (LRc) of Theorem 1.10. Note that at $t=1$, queue 3 is overloaded and, since 2 is at the end of overloading and 1 is at the start of underloading at $t=1,23$ is a critical chain and 21 is a subcritical chain preceding 3, which is the necessary condition stated in (LRc) of Theorem 1.10.

It is significant that the separated discontinuity arises only in the multi-dimensional setting, and not in the one-dimensional setting. This has important ramifications for the mode of convergence of $\nabla_{\chi}^{\varepsilon}(\psi)$ to $\nabla_{\chi} \Gamma(\psi)$ when $\psi, \chi$ are continuous. Specifically, as remarked earlier, it was shown in [25] that for the one-dimensional map, $\nabla_{\chi}^{\varepsilon}(\psi)$ converges to $\nabla_{\chi} \Gamma(\psi)$ in the $M_{1}$ topology [49]. When $\psi, \chi$ are continuous, $\nabla_{\chi}^{\varepsilon}(\psi)$ is also continuous for every $\varepsilon>0$. Since $\mathcal{D}_{l, r}$, the space of functions that are either left or right continuous at every point, is complete under the $M_{1}$ topology [49], and continuous functions clearly lie in $\mathcal{D}_{l, r}$, while functions with separated discontinuities do not lie in $\mathcal{D}_{l, r}$, this example demonstrates that one cannot in general expect $M_{1}$ convergence in the multi-dimensional case. This issue is discussed further in [28], where the mode of convergence is relevant for proving functional central limits for time-inhomogeneous queueing networks.

\section{Existence and Characterization of the Directional Derivative}

This section is devoted to establishing existence of and characterizating the directional derivative for H-R ORPs, as stated in Theorem 1.6. Relevant properties of H-R ORPs with inputs $\psi \in \mathcal{D}_{\text {lim }}$ are first described in Section 3.1, and then existence of the associated directional derivative is established in Section 3.2. In Section 3.3 the notion of a generalized one-dimensional derivative is introduced and characterized, leading to the proof of Theorem 1.6 in Section 3.4. 


\subsection{Properties of the Oblique Reflection Map}

The ORP associated with an H-R matrix $R \in \mathbb{R}^{K \times K}$ was introduced in Section 1.3. Here we first establish a minor generalization of a well-known result of Harrison and Reiman [16] to show that RMs $\Gamma$ associated with $\mathrm{H}-\mathrm{R}$ reflection matrices are well-defined on $\mathcal{D}_{\text {lim }}$. Recall the notation $\bar{f}(t)=\sup _{s \in[0, t]} f(s)$.

Theorem 3.1 (Solutions to H-R ORPs) Given an ORP with $H$-R constraint matrix $R \in$ $\mathbb{R}^{K \times K}, P=I-R$ and $\psi \in \mathcal{D}_{\lim }^{+}$, there exists a unique solution $(\phi, \theta)$ for $\psi$. Moreover, $\theta=\Theta(\psi)$ is the unique fixed point of the map $F(\psi, \cdot): \mathcal{I}_{0} \rightarrow \mathcal{I}_{0}$ given by

$$
F^{i}(\psi, \theta)(t) \doteq\left[\overline{-\psi^{i}+[P \theta]^{i}}(t)\right] \vee 0 \quad \text { for } i=1, \ldots, K
$$

In other words, for $i=1, \ldots, K, \theta^{i}$ satisfies

$$
\theta^{i}(t)=\left[\overline{-\psi^{i}+[P \theta]^{i}}(t)\right] \vee 0
$$

Furthermore, the maps $\Gamma$ and $\Theta$ are Lipschitz continuous with respect to the uniform topology on $\mathcal{D}_{\text {lim }}$, that is, there exists $L=L(R)<\infty$ such that for every $\psi_{1}, \psi_{2} \in \mathcal{D}_{\lim }$ and $N<\infty$,

$$
\left\|\Gamma\left(\psi_{1}\right)-\Gamma\left(\psi_{2}\right)\right\|_{N} \leq L\left\|\psi_{1}-\psi_{2}\right\|_{N} \quad \text { and } \quad\left\|\Theta\left(\psi_{1}\right)-\Theta\left(\psi_{2}\right)\right\|_{N} \leq L\left\|\psi_{1}-\psi_{2}\right\|_{N}
$$

Lastly, if $\psi \in \mathcal{C}\left(\mathcal{D}_{c}\right)$, then $\phi, \theta \in \mathcal{C}$ (respectively, $\left.\mathcal{D}_{c}\right)$.

Proof. It is easy to verify that $F(\psi, \theta) \in \mathcal{I}_{0}$ whenever $(\psi, \theta) \in \mathcal{D}_{\lim }^{+} \times \mathcal{I}_{0}$. Since $\mathcal{D}_{\text {lim }}$ is complete with respect to the sup norm, the same argument as that used in [16] shows that $F(\psi, \cdot)$ is a contraction mapping that maps $\mathcal{I}_{0}$ into $\mathcal{I}_{0}$, and thus has a unique fixed point. The proof of the fact that $\theta$ is a fixed point of $F(\psi, \cdot)$ if and only if $\theta=\Theta(\psi)$. also follows from a straightforward generalization of the corresponding argument used in [16] from $\mathcal{C}$ to $\mathcal{D}_{\text {lim }}^{+}$, and is thus omitted. Lipschitz continuity of the maps $\Gamma$ and $\Theta$ can be deduced from the explicit representation (3.1) for $F^{i}$ and the fact that the matrix $P$ is similar to a matrix whose row sumes are strictly less than 1 (see Lemma 3.3 for similar arguments or [10] for an alternative proof of Lipschitz continuity when $\psi \in \mathcal{D}_{r}$ ). When $\psi \in \mathcal{C}$, the last statement is a consequence of the fact that $\mathcal{C}$ and $\mathcal{D}_{r}$ are closed subspaces of $\mathcal{D}_{\lim }$ (with respect to the topology of uniform convergence). The case when $\psi \in \mathcal{D}_{c}$ is easily verified directly (see, for example, the argument in [9]). 


\subsection{Existence of the Directional Derivative}

In order to show the existence of the derivative or, equivalently, to show the existence of a pointwise limit of the sequence $\nabla_{\chi}^{\varepsilon}(\psi) \in \mathcal{D}_{\text {lim }}$ as $\varepsilon \downarrow 0$, it turns out to be more convenient to work with a closely related sequence $\left\{\gamma_{\varepsilon}(\psi, \chi)\right\}$. This sequence is introduced in Section 3.2.1 and is shown to have a pointwise limit $\gamma(\psi, \chi)$ in Section 3.2.2. In Section 3.2.3 the limit $\gamma(\psi, \chi)$ and the derivative $\nabla_{\chi} \Gamma(\psi)$ are shown to lie in $\mathcal{D}_{\lim }$ and satisfy certain continuity and scaling properties.

\subsubsection{A related sequence $\left\{\gamma_{\varepsilon}\right\}$}

Given an ORP with H-R constraint matrix $R$, let

$$
\gamma_{\varepsilon}(\psi, \chi) \doteq \varepsilon^{-1}[\Theta(\psi+\varepsilon \chi)-\Theta(\psi)]
$$

where $\Theta$ is the mapping introduced after Definition 1.1. Using the fact that $\Gamma(\psi)=\psi+R \Theta(\psi)$ for $\psi \in \mathcal{D}_{\text {lim }}$, along with definition (1.9) of the sequence $\left\{\nabla_{\chi}^{\varepsilon} \Gamma(\psi)\right\}$ one obtains the relation

$$
\nabla_{\chi}^{\varepsilon} \Gamma(\psi)=\chi+R \gamma_{\varepsilon}(\psi, \chi)
$$

Thus, in order to establish existence of the derivative, it clearly suffices to show that the sequence $\gamma_{\varepsilon}(\psi, \chi)$ has a pointwise limit as $\varepsilon \downarrow 0$.

Now fix $\psi, \chi \in \mathcal{D}_{\text {lim. }}$. For conciseness let $\theta \doteq \Theta(\psi)$ and for $\varepsilon>0$, let $\theta_{\varepsilon} \doteq \Theta(\psi+\varepsilon \chi)$ and $\gamma_{\varepsilon} \doteq \gamma_{\varepsilon}(\psi, \chi)$. Then from (3.2) it follows that

$$
\theta^{i}=\left[\overline{-\psi^{i}+[P \theta]^{i}}\right] \vee 0 \quad \text { and } \quad \theta_{\varepsilon}^{i}=\left[\overline{-\psi^{i}-\varepsilon \chi^{i}+\left[P \theta_{\varepsilon}\right]^{i}}\right] \vee 0 .
$$

Adding and subtracting $[P \theta]^{i}$ under the supremum on the right side of the second equality in the above display, one can rewrite

$$
\theta_{\varepsilon}^{i}=\left[\overline{-\xi^{i}-\varepsilon \chi^{i}+\left[P\left(\theta_{\varepsilon}-\theta\right)\right]^{i}}\right] \vee 0
$$

where

$$
\xi^{i} \doteq \psi^{i}-[P \theta]^{i}
$$

Multiplying the difference between (3.7) and (3.6) by $\varepsilon^{-1}$, and using the definitions of $F$ and $\gamma_{\varepsilon}$ in (3.1) and (3.4) respectively, one infers that for $i=1, \ldots, K$

$$
\gamma_{\varepsilon}^{i}=\gamma_{\varepsilon}^{i}(\psi, \chi)=\overline{-\varepsilon^{-1} \xi^{i}-\chi^{i}+\left[P \gamma_{\varepsilon}\right]^{i}} \vee 0-\overline{-\varepsilon^{-1} \xi^{i}} \vee 0
$$




\subsubsection{Pointwise convergence of $\left\{\gamma_{\varepsilon}\right\}$ for H-R ORPs}

In this section some basic properties of the sequences $\left\{\gamma_{\varepsilon}\right\}$ and $\left\{\nabla_{\chi}^{\varepsilon} \Gamma(\psi)\right\}$ are established. The uniform boundedness property proved in Lemma 3.2 shows that for every $t>0$ the sequence $\left\{\gamma_{\varepsilon}(t)\right\}$ must have a convergent subsequence. Lemma 3.3 then establishes an additional monotonicity property that shows that the sequence $\left\{\gamma_{\varepsilon}(\psi, \chi)\right\}$ has a pointwise limit as $\varepsilon \downarrow 0$. Together, for H-R ORPs, these results establish existence of a pointwise limit $\gamma$ of $\left\{\gamma_{\varepsilon}(\psi, \chi)\right\}$ for $\psi, \chi \in \mathcal{D}_{\lim }$.

Lemma 3.2 (Uniform Boundedness) Let $\nabla_{\chi}^{\varepsilon} \Gamma(\psi)$ and $\gamma_{\varepsilon}(\psi, \chi)$ be defined by (1.9) and (3.4) respectively and let $L<\infty$ be the constant that satisfies (3.3). Then for any $\xi, \chi_{1}, \chi_{2} \in \mathcal{D}_{\lim }$ and $T<\infty$, the following inequalities hold:

$$
\begin{array}{ll}
\sup _{\varepsilon>0}\left\|\nabla_{\chi_{1}}^{\varepsilon} \Gamma(\psi)-\nabla_{\chi_{2}}^{\varepsilon} \Gamma(\psi)\right\|_{T} \leq L\left\|\chi_{1}-\chi_{2}\right\|_{T} & \sup _{\varepsilon>0}\left\|\nabla_{\chi}^{\varepsilon} \Gamma(\psi)\right\|_{T} \leq L\|\chi\|_{T} \\
\sup _{\varepsilon>0}\left\|\gamma_{\varepsilon}\left(\psi, \chi_{1}\right)-\gamma_{\varepsilon}\left(\psi, \chi_{2}\right)\right\|_{T} \leq L\left\|\chi_{1}-\chi_{2}\right\|_{T} & \sup _{\varepsilon>0}\left\|\gamma_{\varepsilon}(\psi, \chi)\right\|_{T} \leq L\|\chi\|_{T} .
\end{array}
$$

Proof. The first and third inequalities follow directly from the Lipschitz continuity of the RM as stated in (3.3) and the definitions of $\nabla_{\chi}^{\varepsilon} \Gamma(\psi)$ and $\gamma_{\varepsilon}$ given in (1.9) and (3.4), respectively. The second and fourth bounds follow simply by choosing $\chi_{1}=\chi$ and $\chi_{2}=0$ in the first and third bounds respectively and noting that $\nabla_{0}^{\varepsilon} \Gamma(\psi)=\gamma_{\varepsilon}(\psi, 0)=0$.

Lemma 3.3 (Monotonicity) Given $\psi, \chi \in \mathcal{D}_{\text {lim }}$, let $\gamma_{\varepsilon} \doteq \gamma_{\varepsilon}(\psi, \chi)$ be defined by (3.4). Then for $i=1, \ldots, K, \gamma_{\varepsilon}^{i}$ is monotonically nonincreasing as $\varepsilon \downarrow 0$, so that

$$
0<\varepsilon_{1} \leq \varepsilon_{2} \quad \text { implies } \quad \gamma_{\varepsilon_{1}}^{i}(s)-\gamma_{\varepsilon_{2}}^{i}(s) \leq 0 \quad \text { for } s \in[0, \infty)
$$

Moreover, the family $\left\{\gamma_{\varepsilon}(\psi, \chi)\right\}$ has a pointwise limit $\gamma(\psi, \chi)$, as $\varepsilon \rightarrow 0$.

Proof. Let $0<\varepsilon_{1} \leq \varepsilon_{2}$ and fix $i \in\{1, \ldots, K\}$ and $s \in[0, \infty)$. Using the representation (3.9) for $\gamma_{\varepsilon}^{i}$ and making repeated use of Lemma 6.1, it follows that for $0 \leq \varepsilon_{1} \leq \varepsilon_{2}$ and $t \in[0, s]$,

$$
\begin{aligned}
\gamma_{\varepsilon_{1}}^{i}(t)-\gamma_{\varepsilon_{2}}^{i}(t) & =\overline{-\varepsilon_{1}^{-1} \xi^{i}-\chi^{i}+\left[P \gamma_{\varepsilon_{1}}\right]^{i}}(t) \vee 0-\overline{\varepsilon_{1}^{-1} \xi^{i}}(t) \vee 0 \\
& =\frac{-\overline{-\varepsilon_{2}^{-1} \xi^{i}-\chi^{i}+\left[P \gamma_{\varepsilon_{2}}\right]^{i}}(t) \vee 0+\overline{\varepsilon_{2}^{-1} \xi^{i}}(t) \vee 0}{-\varepsilon_{1}^{-1} \xi^{i}-\chi^{i}+\left[P \gamma_{\varepsilon_{1}}\right]^{i}}(t) \vee 0-\overline{-\varepsilon_{2}^{-1} \xi^{i}-\chi^{i}+\left[P \gamma_{\varepsilon_{2}}\right]^{i}}(t) \vee 0 \\
& \leq \overline{-\left(\varepsilon_{1}^{-1}-\varepsilon_{2}^{-1}\right)\left[\overline{-\xi^{i}}(t) \vee 0\right]} \\
& \leq \overline{\left[P \varepsilon_{1}^{-1}-\varepsilon_{2}^{-1}\right) \xi^{i}+\left[P \gamma_{\varepsilon_{1}}\right]^{i}-\left[P \gamma_{\varepsilon_{2}}\right]^{i}}(t) \vee 0 .
\end{aligned}
$$


By Remark 1.3, there exists a diagonal matrix $A$ with $A_{i i}>0$ for $i=1, \ldots, K$, and $\delta>0$ such that the matrix $\tilde{P} \doteq A^{-1} P A$ satisfies $\max _{i=1, \ldots, K} \sum_{j=1}^{K} \tilde{P}_{i j} \leq 1-\delta$. Define $\tilde{\gamma} \doteq A^{-1} \gamma$. Using the fact that $\tilde{P}$ is nonnegative (since $P$ is nonnegative), we obtain for every $t \in[0, s]$,

$$
\begin{aligned}
\frac{\tilde{\gamma}_{\varepsilon_{1}}^{i}-\tilde{\gamma}_{\varepsilon_{2}}^{i}}{\varepsilon^{2}}(t) & =\frac{1}{A_{i i}}\left[\gamma_{\varepsilon_{1}}^{i}(t)-\gamma_{\varepsilon_{2}}^{i}(t)\right] \\
\leq \quad \frac{1}{A_{i i}} \overline{\left[A \tilde{P} \tilde{\gamma}_{\varepsilon_{1}}\right]^{i}-\left[A \tilde{P} \tilde{\gamma}_{\varepsilon_{2}}\right]^{i}}(s) \vee 0 & =\frac{\left[\tilde{P} \tilde{\gamma}_{\varepsilon_{1}}\right]^{i}-\left[\tilde{P} \tilde{\gamma}_{\varepsilon_{2}}\right]^{i}}{(s) \vee 0} \\
\leq \quad\left(\sum_{j=1}^{K} \tilde{P}_{i j}\right)_{k=1, \ldots, K} \max _{\varepsilon_{1}} \overline{\tilde{\gamma}_{\varepsilon_{1}}^{k}-\tilde{\gamma}_{\varepsilon_{2}}^{k}}(s) \vee 0 & \leq(1-\delta) \max _{k=1, \ldots, K} \overline{\tilde{\gamma}_{\varepsilon_{1}}^{k}-\tilde{\gamma}_{\varepsilon_{2}}^{k}}(s) \vee 0 .
\end{aligned}
$$

Taking the supremum of the left-hand side of the above inequality over $t \in[0, s]$ and then the maximum over $i=1, \ldots, K$ yields the relation

$$
\max _{k=1, \ldots, K} \overline{\tilde{\gamma}_{\varepsilon_{1}}^{k}-\tilde{\gamma}_{\varepsilon_{2}}^{k}}(s) \leq(1-\delta) \max _{k=1, \ldots, K} \overline{\tilde{\gamma}_{\varepsilon_{1}}^{k}-\tilde{\gamma}_{\varepsilon_{2}}^{k}}(s) \vee 0
$$

from which (3.12) follows for $\tilde{\gamma}$, and hence for $\gamma$.

Now the uniform boundedness of the sequence $\left\{\gamma_{\varepsilon}\right\}$ proved in Lemma 3.2 shows that for each $s \in[0, \infty)$, there exists a subsequence (which could depend on $s$ ) of $\left\{\gamma_{\varepsilon}(s)\right\}$ that converges to a limit. The monotonicity property shows that this limit, which we denote by $\gamma(s)$, is independent of the subsequence. Thus $\gamma$ is the pointwise limit of the sequence of functions $\left\{\gamma_{\varepsilon}\right\}$.

\subsubsection{Proof of Theorem 1.6(1)}

In this section we first show that the limit $\gamma$ of Lemma 3.3 lies in $\mathcal{D}_{\text {lim }}$. Note that this is not apriori obvious even if $\psi$ and $\chi$ are assumed to be continuous (which would in turn imply that the functions $\gamma_{\varepsilon}=\gamma_{\varepsilon}(\psi, \chi), \varepsilon>0$, are continuous) since the limit of a monotone non-increasing sequence of real-valued continuous functions $\left\{f_{n}\right\}$ need not in general lie in $\mathcal{D}_{\text {lim }}$. For instance the sequence of continuous functions $\left\{f_{n}\right\}$, where $f_{n}(x)=\sin (1 / x)$ if $x \in[1 /(2 n \pi+\pi / 2), \infty)$ and $f_{n}(x)=1$ otherwise, decreases and converges pointwise to the function $f$ defined by $f(x)=$ $\sin (1 / x)$ if $x \in(0, \infty)$ and $f(0)=1$, which does not lie in $\mathcal{D}_{\text {lim }}$ since it has no right limit at 0 . However, the sequence $\left\{\gamma_{\varepsilon}\right\}$ possesses special properties by virtue of the fact that it is defined via an ORP, which allow one to conclude that $\gamma$ must lie in $\mathcal{D}_{\text {lim }}$. The case when $\chi \in \mathcal{B V}$ is proved in Lemma 3.4 and the general case is dealt with in the proof of Theorem 1.6(1), which is presented after Lemma 3.4. Recall that $|f|_{T}$ denotes the total variation of the function $f$ on the interval $[0, T]$. 
Lemma 3.4 (Uniformly BV) Given an $H$ - $R$ reflection matrix $R$ and associated $R M \Gamma, \psi \in$ $\mathcal{D}_{\lim }$ and $\chi \in \mathcal{B V}$, let $\gamma_{\varepsilon} \doteq \gamma_{\varepsilon}(\psi, \chi)$ be defined by (3.4). Then for every $T \in[0, \infty)$

$$
\sup _{\varepsilon>0}\left|\gamma_{\varepsilon}\right|_{T}<\infty .
$$

Moreover the pointwise limit $\gamma(\psi, \chi)$ of $\gamma_{\varepsilon}(\psi, \chi)$ lies in $\mathcal{D}_{\lim }$.

Proof. Fix $T<\infty$ and let $P=I-R$. Using the representation (3.9) for $\gamma_{\varepsilon}^{i}$, Lemma 6.1 and the fact that $P$ is non-negative it follows that for $\varepsilon>0$

$$
\begin{aligned}
\left|\gamma_{\varepsilon}^{i}\right|_{\mathrm{T}} & =\left|\overline{-\varepsilon^{-1} \xi^{i}-\chi^{i}+\left[P \gamma_{\varepsilon}\right]^{i}} \vee 0-\overline{-\varepsilon^{-1} \xi^{i}} \vee 0\right|_{\mathrm{T}} \\
& \leq\left|-\chi^{i}+\left[P \gamma_{\varepsilon}\right]^{i}\right|_{\mathrm{T}} \\
& \leq\left|\chi^{i}\right|_{\mathrm{T}}+\sum_{j=1}^{K} P_{i j}\left|\gamma_{\varepsilon}^{j}\right|_{T V} .
\end{aligned}
$$

By Remark 1.3 there exists a diagonal matrix $A$ (with $A_{i i}>0$ ) and $\delta>0$ such that the matrix $\tilde{P} \doteq A^{-1} P A$ satisfies $\max _{i=1, \ldots, K} \sum_{j=1}^{K} \tilde{P}_{i j} \leq 1-\delta$. Multiplying both sides of the last display by $A_{i i}$ and substituting for $P$ in terms of $\tilde{P}$ (note that $A_{j j} \tilde{P}_{i j}=A_{i i} P_{i j}$ ), we obtain the inequality

$$
A_{i i}\left|\gamma_{\varepsilon}^{i}\right|_{\mathrm{T}} \leq A_{i i}\left|\chi^{i}\right|_{\mathrm{T}}+\sum_{j=1}^{K} \tilde{P}_{i j} A_{j j}\left|\gamma_{\varepsilon}^{j}\right|_{\mathrm{T}},
$$

which implies that

$$
\max _{i=1, \ldots, K} A_{i i}\left|\gamma_{\varepsilon}^{i}\right|_{\mathrm{T}} \leq \max _{i=1, \ldots, K} A_{i i}\left|\chi^{i}\right|_{\mathrm{T}}+(1-\delta) \max _{i=1, \ldots, K} A_{i i}\left|\gamma_{\varepsilon}^{i}\right|_{\mathrm{T}}
$$

This can in turn be rearranged to obtain

$$
\max _{i=1, \ldots, K} A_{i i}\left|\gamma_{\varepsilon}^{i}\right|_{\mathrm{T}} \leq \frac{\max _{i=1, \ldots, K} A_{i i}\left|\chi^{i}\right|_{\mathrm{T}}}{\delta}
$$

from which we conclude that

$$
\sup _{\varepsilon>0}\left|\gamma_{\varepsilon}\right|_{\mathrm{T}} \leq K \sup _{\varepsilon>0} \max _{i=1, \ldots, K}\left|\gamma_{\varepsilon}^{i}\right|_{\mathrm{T}} \leq \frac{K \max _{i=1, \ldots, K} A_{i i}}{\delta \min _{i=1, \ldots, K} A_{i i}}|\chi|_{\mathrm{T}}<\infty
$$

where the last inequality follows because of the assumption that $\chi \in \mathcal{B V}$. Combining this property with the fact that the pointwise limit $\gamma$ of the family of functions $\left\{\gamma_{\varepsilon}\right\}$ is uniformly bounded (due to (3.11)) and Lemma 6.2, it follows that $\gamma \in \mathcal{D}_{\text {lim. }}$.

Proof of Theorem 1.6(1). For any $\psi, \chi \in \mathcal{D}_{\text {lim }}$, Lemma 3.3 establishes the existence of the limit $\gamma(\psi, \chi)$. Moreover, if $\chi \in \mathcal{B V}$, Lemma 3.4 shows that $\gamma(\psi, \chi) \in \mathcal{D}_{\text {lim }}$. Now let $\chi \in \mathcal{D}_{\lim }$. 
Since $\mathcal{D}_{c, l i m}$ is dense in $\mathcal{D}_{\text {lim }}$ with respect to the topology of uniform convergence on compact sets (see, for example, [49]) and clearly, $\mathcal{D}_{c, \text { lim }} \subset \mathcal{B V}$, in particular $\mathcal{B V}$ is dense in $\mathcal{D}_{\text {lim }}$. Thus $\chi$ can be expressed as the limit (in the uniform norm) of a sequence $\left\{\chi_{n}\right\} \subset \mathcal{B V}$, and from Lemma 3.2 it follows that $\gamma\left(\psi, \chi_{n}\right)$ converges to $\gamma(\psi, \chi)$ in the uniform norm. Since $\gamma\left(\psi, \chi_{n}\right) \in \mathcal{D}_{\text {lim }}$ for every $n$, and $\mathcal{D}_{\text {lim }}$ is complete, we deduce that $\gamma(\psi, \chi) \in \mathcal{D}_{\text {lim }}$. The relation (3.5) then shows that the pointwise limit $\nabla_{\chi} \Gamma(\psi)$ of $\nabla_{\chi}^{\varepsilon} \Gamma(\psi)$ exists and is equal to $\chi+R \gamma$, from which it automatically follows that $\nabla_{\chi} \Gamma(\psi) \in \mathcal{D}_{\text {lim }}$.

For any fixed $\psi \in \mathcal{D}_{\text {lim }}$, the Lipschitz continuity of $\nabla . \Gamma(\psi)$ with respect to $\chi$ is a direct consequence of (3.10). Lastly, since $\nabla_{\chi} \Gamma(\psi)=\chi+R \gamma(\psi, \chi)$, in order to establish (1.15) it suffices to show that for $\alpha, \beta>0, \gamma(\beta \psi, \alpha \chi)=\alpha \gamma(\psi, \chi)$. From (3.2) it is clear that for $\beta>0$, $\Theta(\beta \psi)=\beta \Theta(\psi)$. Fix $\varepsilon>0$. By (3.4) we then see that

$$
\gamma_{\varepsilon}(\beta \psi, \alpha \chi)=\varepsilon^{-1}[\Theta(\beta \psi+\varepsilon \alpha \chi)-\Theta(\beta \psi)]=\beta \varepsilon^{-1}\left[\Theta\left(\psi+\varepsilon \frac{\alpha}{\beta} \chi\right)-\Theta(\psi)\right] .
$$

Setting $\tilde{\varepsilon}=\varepsilon \alpha / \beta$, we can rewrite the above equation as

$$
\gamma_{\varepsilon}(\beta \psi, \alpha \chi)=\alpha \tilde{\varepsilon}^{-1}[\Theta(\psi+\tilde{\varepsilon} \chi)-\Theta(\psi)]=\alpha \gamma_{\tilde{\varepsilon}}(\psi, \chi)
$$

Taking limits as $\varepsilon \rightarrow 0$, and noting that then $\tilde{\varepsilon} \rightarrow 0$, we obtain the desired relation $\gamma(\beta \psi, \alpha \chi)=$ $\alpha \gamma(\psi, \chi)$

\subsection{The Generalized One-dimensional Derivative}

\subsubsection{A representation for $\gamma^{i}$}

In the last section we showed that given $\psi, \chi \in \mathcal{D}_{\text {lim }}$, the directional derivative has the form $\nabla_{\chi} \Gamma(\psi)=\chi+R \gamma$, where $\gamma \doteq \gamma(\psi, \chi) \in \mathcal{D}_{\text {lim }}$ is the pointwise limit of the monotonically nonincreasing sequence $\left\{\gamma_{\varepsilon}(\psi, \chi)\right\}$. From the expression (3.9) for $\gamma_{\varepsilon}^{i} \doteq \gamma_{\varepsilon}^{i}(\psi, \chi)$, it is clear that for every $t \in[0, \infty)$,

$$
\begin{aligned}
\gamma^{i}(t) & =\lim _{\varepsilon \downarrow 0}\left[\overline{-\varepsilon^{-1} \xi^{i}-\chi^{i}+\left[P \gamma_{\varepsilon}\right]^{i}}(t) \vee 0-\overline{-\varepsilon^{-1} \xi^{i}}(t) \vee 0\right] \\
& =\lim _{\varepsilon \downarrow 0}\left[F^{i}\left(\varepsilon^{-1} \xi+\chi-P \gamma_{\varepsilon}, 0\right)(t)-F^{i}\left(\varepsilon^{-1} \xi, 0\right)(t)\right] .
\end{aligned}
$$

Since $P$ is non-negative, it follows that $P \gamma$ is the pointwise limit of the monotonically nonincreasing sequence $\left\{P \gamma_{\varepsilon}\right\}$. Thus $\gamma^{i}$ has a representation as a one-dimensional pointwise limit of the form

$$
\lim _{\varepsilon \downarrow 0}\left[\overline{\varepsilon^{-1} f+g_{\varepsilon}} \vee 0-\overline{\varepsilon^{-1} f} \vee 0\right]
$$


where $f, g_{\varepsilon} \in \mathcal{D}_{\text {lim }}$ and $g_{\varepsilon}$ monotonically converges pointwise down to a function $g \in \mathcal{D}_{\text {lim }}$ as $\varepsilon \downarrow 0$ (indeed, make the identification $f \doteq-\xi^{i}, g_{\varepsilon} \doteq-\chi^{i}+\left[P \gamma_{\varepsilon}\right]^{i}$ and $\left.g=-\chi^{i}+[P \gamma]^{i}\right)$. If for every $\varepsilon>0, g_{\varepsilon} \equiv g$ were independent of $\varepsilon$, then (3.15) would reduce to a limit of the form

$$
\lim _{\varepsilon \downarrow 0}\left[\overline{\left(\varepsilon^{-1} f+g\right.} \vee 0-\overline{\varepsilon^{-1} f} \vee 0\right]
$$

Under the assumption that $f, g \in \mathcal{C}$ and the limit has a finite number of discontinuities on any compact interval, the limit in (3.16) was shown in [25] to be equal to $\max _{s \in \Phi_{f}(t)} g(s)$, where $\Phi_{f}$ is as defined in (1.11). This representation was later generalized to the case $f, g \in \mathcal{D}_{r}$ in [50, Theorem 9.3.1]. One may be tempted to conjecture that the limit in (3.15) is equal to the limit in (3.16) with $g \in \mathcal{D}_{\text {lim }}$ equal to the pointwise limit of $\left\{g_{\varepsilon}\right\}$. If that were true, then the limit in (3.15) could be identified simply by generalizing the results in $[25,50]$ to the case when $f, g \in \mathcal{D}_{\text {lim }}$. However, it turns out that the topology of pointwise convergence $g_{\varepsilon} \downarrow g$ is too weak for such a conjecture to hold in general (see Remark 3.6(3) for examples when the two limits fail to coincide). Thus a more careful analysis is required in order to determine the correct limit in (3.15). This is carried out in Section 3.3.2. Fortunately, it turns out that the conjecture is true for the special case when $f \in \mathcal{C}$ and $g_{\varepsilon} \in \mathcal{C}$ for all $\varepsilon>0$, and in this case the one-dimensional limit takes a rather nice form (see Theorem 3.5). In the multi-dimensional case, when $P \neq 0$, (3.14) leads to a finite system of coupled equations that implicitly determine $\gamma$. The additional justification required to establish that this system of equations uniquely determines $\gamma$ (under suitable conditions on $\psi$ and $\chi$ ) is provided in Section 3.4.

\subsubsection{Identification of the generalized one-dimensional derivative}

In order to describe the limit in (3.15) we need to first introduce some definitions. For $f, g, g_{1}, g_{2} \in$ $\mathcal{D}_{\text {lim }}$, define

$$
H\left(f, g, g_{1}, g_{2}\right)(t) \doteq \begin{cases}0 & \text { for } t \in\left(0, t_{\ell}\right), \\ S\left(f, g, g_{1}, g_{2}\right)(t) \vee 0 & \text { for } t \in\left[t_{l}, t_{u}\right], \\ S\left(f, g, g_{1}, g_{2}\right)(t) & \text { for } t \in\left(t_{u}, \infty\right)\end{cases}
$$

where

$$
\begin{aligned}
& t_{\ell}=t_{\ell}(f) \doteq \inf \{t>0: \bar{f}(t)=0\} \\
& t_{u}=t_{u}(f) \doteq \sup \{t>0: \bar{f}(t)=0\}
\end{aligned}
$$


and

$$
S\left(f, g, g_{1}, g_{2}\right)(t) \doteq \sup _{s \in \Phi_{f}^{L}(t)}\left\{g_{1}(s)\right\} \vee \sup _{s \in \Phi_{f}(t)}\{g(s)\} \vee \sup _{s \in \tilde{\Phi}_{f}^{R}(t)}\left\{g_{2}(s)\right\}
$$

with $\Phi_{f}, \Phi_{f}^{L}$ and $\tilde{\Phi}_{f}^{R}$ defined as in (1.11), (1.12) and (1.13), respectively. Moreover, let

$$
S_{1}(f, g) \doteq S(f, g, g, g) \quad \text { and } \quad H_{1}(f, g) \doteq H(f, g, g, g)
$$

and, likewise, let

$$
S_{2}(f, g) \doteq S\left(f, g, g_{l}, g_{r}\right) \quad \text { and } \quad H_{2}(f, g) \doteq H\left(f, g, g_{l}, g_{r}\right)
$$

where $g_{l}$ and $g_{r}$ are the left and right regularizations of $g$, as defined in (1.6). It is easy to see that for $f \in \mathcal{D}_{\lim }$ and $t \in[0, \infty), \Phi_{f}^{L}(t) \cup \Phi_{f}(t) \cup \tilde{\Phi}_{f}^{R}(t) \neq \emptyset$ and hence $S\left(f, g, g_{1}, g_{2}\right), S_{1}(f, g)$ and $S_{2}(f, g)$ are always finite. The following theorem provides a useful characterization of the generalized one-dimensional derivative.

Theorem 3.5 (Generalization of the one-dimensional derivative) Consider a sequence $\left\{g_{\varepsilon}\right\} \subseteq \mathcal{D}_{\text {lim }}(\mathbb{R})$ such that $\sup _{\varepsilon>0}\left\|g_{\varepsilon}\right\|_{N}<\infty$ for every $N \in[0, \infty)$ and for every $s \in[0, \infty)$

$$
\varepsilon_{1} \leq \varepsilon_{2} \quad \Rightarrow \quad g_{\varepsilon_{1}}(s) \leq g_{\varepsilon_{2}}(s)
$$

Moreover, let $g, g^{*, l}, g^{*, r} \in \mathcal{D}_{\lim }(\mathbb{R})$ be such that $g_{\varepsilon} \downarrow g \in \mathcal{D}_{\lim }(\mathbb{R}), g_{\varepsilon, l} \downarrow g^{*, l}$ and $g_{\varepsilon, r} \downarrow g^{*, r}$ pointwise as $\varepsilon \downarrow 0$, where $g_{\varepsilon, l}$ and $g_{\varepsilon, r}$ are respectively the left and right regularizations of $g_{\varepsilon}$. For $f \in \mathcal{D}_{\lim }(\mathbb{R})$, if

$$
\tilde{\gamma}_{\varepsilon} \doteq \overline{\varepsilon^{-1} f+g_{\varepsilon}} \vee 0-\overline{\varepsilon^{-1} f} \vee 0
$$

then $\tilde{\gamma}_{\varepsilon} \rightarrow \tilde{\gamma} \in \mathcal{D}_{\text {lim }}(\mathbb{R})$ pointwise as $\varepsilon \downarrow 0$, where

$$
\tilde{\gamma} \doteq H\left(f, g, g^{*, l}, g^{*, r}\right)
$$

and $H$ is given by (3.17). Moreover, if $\left\{g_{\varepsilon}, \varepsilon>0\right\} \subset \mathcal{C}$ then the generalized derivative takes the simpler form

$$
\tilde{\gamma}=H_{1}(f, g)
$$

and if in addition $f \in \mathcal{C}$, then $\tilde{\gamma}=H_{1}(f, g)=H_{2}(f, g)$ and

$$
S_{1}(f, g)=S_{2}(f, g)=\sup _{s \in \Phi_{f}(t)}[g(s)] .
$$

Lastly, if $f \in \mathcal{D}_{c}$ and $g \in \mathcal{D}_{\text {lim }}$ then

$$
\tilde{\gamma}=H_{2}(f, g)
$$


The proof of Theorem 3.5 is relegated to Section 5. Here we make some observations on the theorem.

Remark 3.6 (The generalized one-dimensional derivative)

1. Note that for $f, g \in \mathcal{D}_{\text {lim }}$, the limit in (3.16) is equal to the function $H_{2}(f, g)$ defined in (3.22). Indeed, when $g_{\varepsilon}=g$ is independent of $\varepsilon$, then clearly $g^{*, l}=g_{l}$ and $g^{*, r}=g_{r}$, and so in this case by $(3.22)$ we have $H\left(f, g, g^{*, l}, g^{*, r}\right)=H\left(f, g, g_{l}, g_{r}\right)=H_{2}(f, g)$. If in addition $f, g \in \mathcal{C}$, then $\Phi_{f}^{L}(t) \cup \Phi_{f}(t) \cup \Phi_{f}^{R}(t)=\Phi_{f}(t)$ and $g(s-)=g(s)=g(s+)$, so that

$$
S_{2}(f, g)(t)=\sup _{s \in \Phi_{f}(t)}[g(s)]
$$

Thus Theorem 3.5 contains as a special case the results in [25, Lemma 5.2]. and [50, Theorem 9.3.1].

2. Note that the notation $\tilde{\Phi}_{f}^{R}$ rather than $\Phi_{f}^{R}$ is used in the definitions of $S, S_{1}$ and $S_{2}$ in order to emphasize that $t \notin \tilde{\Phi}_{f}^{R}(t)$, in contrast with the sets $\Phi_{f}^{L}(t)$ and $\Phi_{f}(t)$, which could contain $t$. In the definition for $S_{2}(f, g)$ in [50, Theorem 9.3.1], however, the set $\tilde{\Phi}_{f}^{R}$ is replaced by the set

$$
\Phi_{f}^{R}(t) \doteq\{s \in[0, t]: f(s+)=\bar{f}(t)\},
$$

which could contain $t$. This gives the correct expression when $g \in \mathcal{D}_{r}$, which is the setting considered in [50]. However, the following example shows that when $g \in \mathcal{D}_{\text {lim }}$, even if $f \in \mathcal{C}$, the correct definition of $S_{2}$ is with $\tilde{\Phi}_{f}^{R}$ rather than with $\Phi_{f}^{R}$.

Example 1. Let $f(s) \doteq s 1_{[0,1)}(s)+1_{[1,2)}(s)$ for $s \in[0,2]$, and for every $\varepsilon>0$, let $g_{\varepsilon}(s)=$ $g(s) \doteq 1_{(1,2]}$ for $s \in[0,2]$. Then $f$ is continuous and $g$ is left continuous. Moreover, from the definition of $f$ it follows that $\Phi_{f}^{L}(1)=\Phi_{f}(1)=\{1\}$ and $\tilde{\Phi}_{f}^{R}(1)=\emptyset$, while $\Phi_{f}^{R}(1)=\{1\}$. By (1.22) we have $S_{2}(f, g)(1)=g(1-) \vee g(1)=0$, while for the modified case (i.e. with $\tilde{\Phi}_{f}^{R}$ replaced by $\Phi_{f}^{R}$ in the definition of $\left.S_{2}\right)$ we see that $S_{2}(f, g)(1)=g(1-) \vee g(1) \vee g(1+)=1$. However, by direct verification it is easy to see in this simple example that

$$
\lim _{\varepsilon \downarrow 0}\left[\overline{\varepsilon^{-1} f+g_{\varepsilon}}(1)-\overline{\varepsilon^{-1} f}(1)\right]=\lim _{\varepsilon \downarrow 0}\left[\overline{\varepsilon^{-1} f+g}(1)-\overline{\varepsilon^{-1} f}(1)\right]=g(1)=0 .
$$

3. When both $f$ and $\left\{g_{\varepsilon}, \varepsilon>0\right\}$ are continuous and $g_{\varepsilon} \downarrow g$, it follows from (3.25) and (3.26) that $\tilde{\gamma}=H_{1}(f, g)=H_{2}(f, g)$. Since the limit in (3.15) is given by $\tilde{\gamma}$ and, by Remark 3.6.1 above, the limit in (3.16) is given by $\mathrm{H}_{2}(f, g)$, we see that the two limits in (3.15) and 
(3.16) coincide in this special case. However, the following two examples demonstrate that these two limits need not be equal for general $f, g,\left\{g_{\varepsilon}\right\} \in \mathcal{D}_{\text {lim }}$. Example 2 demonstrates the necessity of having $g_{\varepsilon}$ continuous, while Example 3 shows why $f$ must be continuous.

Example 2. Let $f(s) \doteq s$ and $g(s) \doteq 1$ for $s \in[0,2]$. Also, for $\varepsilon>0$, let

$$
g_{\varepsilon} \doteq \begin{cases}1 & \text { for } t \in[0,1-\varepsilon) \\ 2 & \text { for } t \in[1-\varepsilon, 1) \\ 1 & \text { for } t \in[1,2] .\end{cases}
$$

Then clearly $f$ and $g$ are continuous and each $g_{\varepsilon}$ is right continuous. Moreover, $\Phi_{f}^{L}(1)=$ $\Phi_{f}(1)=1, \tilde{\Phi}_{f}^{R}(1)=\emptyset$ and the fact that $g_{\varepsilon}(1-)=2$ for every $\varepsilon>0$ implies $g^{*, l}(1)=2$. By Theorem 3.5 the limit in (3.15) is equal to $S\left(f, g, g^{*, l}, g^{*, r}\right)=g^{*, l}(1) \vee g(1)=2$, while by Remark 3.6.1 above the limit in (3.16) is equal to $S_{2}(f, g)=g(1-) \vee g(1)=1 \neq 2$.

Example 3. On the interval $[0,2]$ define the functions $f(s) \doteq s 1_{[0,1)}, g \doteq 1_{[1,2]}$ and

$$
g_{\varepsilon}(s) \doteq\left\{\begin{aligned}
0 & \text { for } s \in[0,1-\varepsilon) \\
\frac{s-(1-\varepsilon)}{\varepsilon} & \text { for } s \in[1-\varepsilon, 1) \\
1 & \text { for } s \in[1,2]
\end{aligned}\right.
$$

Then clearly $\left\{g_{\varepsilon}, \varepsilon>0\right\}$ is a sequence of continuous functions that converges pointwise monotonically down to the right continuous function $g$. Moreover, $f$ is also right continuous and $\Phi_{f}^{L}(1)=\{1\}$ and $\Phi_{f}(1)=\tilde{\Phi}^{R}(1)=\emptyset$. By Remark 3.6.1 above, the limit in (3.16) is given by $S_{2}(f, g)(1)=g(1-)=0$. On the other hand, since $g_{\varepsilon}$ are continuous, by $(3.25)$ of Theorem 3.5, the limit in (3.15) is equal to $S_{1}(f, g)=g(1)=1 \neq 0$.

\subsection{Proof of the First Main Result}

In this section we present the proof of Theorem 1.6. First, we use the explicit representations for the generalized one-dimensional derivative established in Theorem 3.5, along with the monotonic pointwise convergence of $\gamma_{\varepsilon}$ to $\gamma$ established in Section 3.2.2, to obtain autonomous characterizations of $\gamma$ when either $\psi, \chi \in \mathcal{C}$ or when $\psi \in \mathcal{D}_{c}$ and $\chi \in \mathcal{D}_{\lim }$.

Fix $\psi, \chi \in \mathcal{D}_{\text {lim }}$, and as usual let $\gamma_{\varepsilon}$ and $\xi$ be defined via (3.4) and (3.8) respectively. Also, for $i=1, \ldots, K$, let $\chi_{l}^{i}$ and $\chi_{r}^{i}$ be the left and right regularizations of $\chi^{i}$, let $\gamma^{*, l}$ and $\gamma^{*, r}$ be the limits of the left and right regularized sequences $\left\{\gamma_{\varepsilon, l}\right\}$ and $\left\{\gamma_{\varepsilon, r}\right\}$ respectively, and let $\gamma$ be the pointwise limit of $\left\{\gamma_{\varepsilon}\right\}$ (which was shown to exist at the end of Section 3.2). In addition, let 
the functions $H$ and $H_{j}, j=1,2$, be defined as in Section 3.3.2. Combining the relation (3.14) with Theorem 3.5, it follows that for $i=1, \ldots, K$,

$$
\gamma^{i}(\psi, \chi)(t)=H\left(-\xi^{i},-\chi^{i}+[P \gamma]^{i},-\chi_{l}^{i}+\left[P \gamma^{*, l}\right]^{i},-\chi_{r}^{i}+\left[P \gamma^{*, r}\right]^{i}\right)(t)
$$

Since in general $\gamma^{*, l}$ and $\gamma^{*, r}$ depend on the structure of the sequence $\left\{\gamma_{\varepsilon}(\psi, \chi\}\right.$, and are therefore not uniquely determined by $\gamma(\psi, \chi)$, this does not lead to an autonomous characterization of $\gamma(\psi, \chi)$. However, we now show that under additional assumptions on $\psi$ and $\chi, \gamma^{*, l}$ and $\gamma^{*, r}$ are uniquely determined by $\gamma(\psi, \chi)$. Specifically, consider the case when $\psi, \chi \in \mathcal{C}$. Then, by Theorem 3.1, $\theta, \theta_{\varepsilon} \in \mathcal{C}$ and consequently $-\xi$ and $\gamma^{\varepsilon} \in \mathcal{C}$. Likewise, if $\psi \in \mathcal{D}_{c}$ and $\chi \in \mathcal{D}_{\text {lim }}$, it follows from Theorem 3.1 that $\xi \in \mathcal{D}_{c}$. So it follows from Theorem 3.5 that $\gamma \doteq \gamma(\psi, \chi)$ satisfies

$$
\gamma^{i}=H_{j}\left(-\xi^{i},-\chi^{i}+[P \gamma]^{i}\right) \quad \text { for } \quad i=1, \ldots, K
$$

with $j=1$ when $\psi, \chi \in \mathcal{C}$ and $j=2$ when $\psi \in \mathcal{D}_{c}$ and $\chi \in \mathcal{D}_{\text {lim. }}$.

Lemma 3.7 Given an $H$ - $R$ matrix $R$ and $P \doteq I-R$, and $\psi, \chi \in \mathcal{D}_{\text {lim }}$, for $j=1,2$, the system of equations (3.31) has a unique solution $\gamma_{(j)} \doteq \gamma_{(j)}(\psi, \chi) \in \mathcal{D}_{\lim }$. Moreover, for $j=1,2$, given any $\gamma_{0, j} \in \mathcal{D}_{\text {lim }}$, if the sequence $\left\{\gamma_{n, j}\right\}$ is defined recursively by

$$
\gamma_{n+1, j} \doteq H_{j}\left(-\xi^{i},-\chi^{i}+\left[P \gamma_{n, j}\right]^{i}\right)
$$

then for every $N<\infty,\left\|\gamma_{(j)}-\gamma_{n, j}\right\|_{N} \rightarrow 0$ as $n \uparrow \infty$.

Proof. Fix $\psi, \chi \in \mathcal{D}_{\text {lim }}$, and $N<\infty$ and recall from Lemma 3.2 that $\gamma(\psi, \chi)$ is uniformly bounded on $[0, N]$ in $\mathcal{D}_{\lim }$ (with respect to the sup norm). From the definition of $H$ it is easy to see that $H$ maps bounded sets to bounded sets. We show below that $H_{1}$ is a contraction mapping (with respect to the sup norm topology) on $\mathcal{D}_{\text {lim }}$. Since $\mathcal{D}_{\text {lim }}$ endowed with the sup norm metric is a complete metric space, the existence of a unique fixed point for $H_{1}$ then follows from standard theorems [47, Theorems 5.2.1 and 5.2.3].

To establish the contraction property we first consider the case when the maximum row sum of the matrix $P$ is equal to $1-\delta<1$. The general case can then be handled in the usual way using diagonal similarity transforms (see, for example, the proof of Lemma 3.4). Let $\gamma_{1}, \gamma_{2} \in \mathcal{D}_{\text {lim }}$. 
Then the definition of $H_{1}$ along with Lemma 6.1 yields

$$
\begin{aligned}
\max _{i=1, \ldots, K} \sup _{t \in[0, N]} \mid H_{1}^{i}\left(-\xi^{i}\right. & \left.,-\chi^{i}+\left[P \gamma_{1}\right]^{i}\right)-H_{1}^{i}\left(-\xi^{i},-\chi^{i}+\left[P \gamma_{2}\right]^{i}\right) \mid \\
& \leq \sup _{s \in[0, N]} \max _{i=1, \ldots, K}\left|\left[P \gamma_{1}\right]^{i}(s)-\left[P \gamma_{2}\right]^{i}(s)\right| \\
& \leq \sup _{s \in[0, N]} \max _{i=1, j \ldots, K}\left|\sum_{k=1}^{K} P_{i k}\right|\left|\gamma_{1}^{j}(s)-\gamma_{2}^{j}(s)\right| \\
& \leq(1-\delta) \max _{j=1, \ldots, K} \sup _{s \in[0, N]}\left|\gamma_{1}^{j}(s)-\gamma_{2}^{j}(s)\right|,
\end{aligned}
$$

which proves the contraction property since $1-\delta<1$. The proof for $H_{2}$ follows analogously and is thus omitted.

We now establish the equivalence between the sets $\Phi^{i}$ and $\Phi_{-\xi^{i}}$ defined in (1.11) and (1.14), respectively.

Lemma 3.8 Given an $H$ - $R$ reflection matrix $R \in \mathbb{R}^{K \times K}$ and $\psi, \chi \in \mathcal{C}$, let $(\phi, \theta)$ solve the associated ORP for $\psi$, and let $\xi, t_{l}, t_{u}, \Phi_{f}$ and $\Phi^{i}$ be as defined in (3.8), (3.18), (3.19), (1.11) and (1.14), respectively. Then for $i=1, \ldots, K, t_{l}^{i}=t_{l}\left(-\xi^{i}\right)$ if and only if $t_{l}^{i}$ satisfies (1.18), $t_{u}^{i}=t_{u}\left(-\xi^{i}\right)$ if and only if $t_{u}^{i}$ satisfies (1.19) and for $t \in\left[t_{l}^{i}, \infty\right), \Phi^{i}(t)=\Phi_{-\xi^{i}}(t)$.

Proof. In this proof we will make repeated of the fact (which follows from Theorem 3.1) that

$$
\phi^{i}(t)=\psi^{i}(t)+[R \theta]^{i}(t)=\psi^{i}(t)-[P \theta]^{i}(t)+\theta^{i}(t)=\xi^{i}(t)+\overline{-\xi^{i}}(t) \vee 0 .
$$

By definition (3.18) of $t_{l}$, we have $-\xi^{i}(s)<0$ for $s<t_{l}^{i} \doteq t_{l}\left(-\xi^{i}\right)$. Moreover, since $\xi \in \mathcal{C}$, $\xi^{i}\left(t_{l}^{i}\right)=0$. From the above display, it then follows that $t_{l}^{i}$ is characterized by the fact that $\phi^{i}(s)=\xi^{i}(s)>0$ for $s<t_{l}^{i}$ and $\phi^{i}\left(t_{l}^{i}\right)=0$, which proves that $t_{l}^{i}$ can equivalently be defined by (1.18). Now let $t_{u}^{i} \doteq t_{u}\left(-\xi^{i}\right)$ and note that for $s \in\left[0, t_{u}^{i}\right], \theta^{i}(s)=\overline{-\xi^{i}}(s) \vee 0=0$, and therefore for every $s \in\left[0, t_{u}^{i}\right), \theta^{i}$ is flat to the right of $s$. On the other hand, by the definition of $t_{u}$, there must exist a sequence $s_{n} \downarrow t_{u}^{i}$ with $-\xi^{i}\left(s_{n}\right)>-\xi^{i}\left(t_{u}^{i}\right)=0$, and so $\theta^{i}$ is not flat to the right of $s$. This shows that $t_{u}^{i}$ can equivalently be defined by (1.19).

Now for $t>t_{l}^{i}, \overline{-\xi^{i}}(t)>0$. Hence we have $\phi^{i}(t)=\xi^{i}(t)+\overline{-\xi^{i}}(t)$, and for $s<t$, $\phi^{i}(s)=\xi^{i}(s)+\overline{-\xi^{i}}(s)$. Suppose $s \in \Phi_{-\xi^{i}}(t)$, so that $-\xi^{i}(s)=\overline{-\xi^{i}}(t)$. Since $s<t$, this means $-\xi^{i}(s)=\overline{-\xi^{i}}(s)=\overline{-\xi^{i}}(t)$. It immediately follows that $\theta^{i}(s)=\theta^{i}(t)$ and $\phi^{i}(s)=$ $\xi^{i}(s)+\overline{-\xi^{i}}(s)=-\overline{-\xi^{i}}(s)+\overline{-\xi^{i}}(s)=0$, and so $s \in \Phi^{i}(t)$. To show the converse, suppose $s \in \Phi^{i}(t)$. Then $\theta^{i}(s)=\theta^{i}(t)$, which implies $\overline{-\xi^{i}}(s)=\overline{-\xi^{i}}(t)$. Moreover $\phi^{i}(s)=0$, which implies $\xi^{i}(s)=-\overline{-\xi^{i}}(s)$. When combined, the last two statements show that $-\xi^{i}(s)=\overline{-\xi^{i}}(t)$, so that 
$s \in \Phi_{-\xi^{i}}(t)$, and the proof of the lemma is complete.

Proof of Theorem 1.6. Property (1) of Theorem 1.6 was proved at the end of Section 3.2.3, where it was also shown that for $\psi, \chi \in \mathcal{D}_{\lim }, \nabla_{\chi} \Gamma(\psi)=\chi+R \gamma$. Now suppose $\psi, \chi \in \mathcal{C}$. Then we have $\xi, \gamma^{\varepsilon}(\psi, \chi) \in \mathcal{C}$ and so Lemma 3.7 shows that $\gamma(\psi, \chi)=\gamma_{(1)}$, where $\gamma_{(1)}$ is the unique solution in $\mathcal{D}_{\text {lim }}$ to the system of equations (3.31) with $j=1$. The relation for $S_{1}$ in (3.26) of Theorem 3.5, along with Lemma 3.8, then shows that the system of equations (3.31) coincides with the system of equations (1.17), and thus property (2) is proved. Similarly, when $\psi \in \mathcal{D}_{c}$ and $\chi \in \mathcal{D}_{\text {lim }}$, Lemma 3.7 shows that $\gamma(\psi, \chi)=\gamma_{(2)}$, where $\gamma_{(2)}$ is the unique solution to the system of equations (3.31) with $j=2$. However, once again by Lemma 3.8, the system of equations (3.31) is identical to the system (1.21) due to the corresponding equivalence of the relations for $S_{2}$ given in (3.22) and (1.22). This completes the proof of Property (3), and therefore of the theorem.

\section{Discontinuities of $\nabla_{\chi} \Gamma(\psi)$ when $\psi, \chi \in \mathcal{C}$}

Throughout this section we fix an ORP with an H-R constraint matrix $R \in \mathbb{R}^{K \times K}$ and $\psi, \chi \in \mathcal{C}$ and, as usual, let $\xi^{i}$ be defined as in (3.8). For conciseness, we denote the corresponding unique solution $\gamma_{(1)}$ to the set of equations (1.17) simply by $\gamma$. The main result of this section is the proof of Theorem 1.10, which is presented in Section 4.3. First, in Section 4.1 we derive necessary and sufficient conditions for the existence of discontinuities in $\gamma^{i}$ in terms of properties

of the set $\Phi_{-\xi^{i}}$. In Section 4.2, we then establish equivalences between properties of $\Phi_{-\xi^{i}}$ and regimes introduced in Definition 1.8, which provide a more physically intuitive description of when discontinuities occur.

\subsection{Classification of the Discontinuities of $\gamma_{(1)}$}

The main result of this section is Theorem 4.2, which provides necessary and sufficient conditions for the existence of discontinuities in $\gamma^{i}$ in terms of properties of the set functions $\Phi_{-\xi^{i}}(\cdot)$. We first introduce some additional notation. For $i=1, \ldots, K$, define

$$
\mathcal{A}^{i} \doteq\left\{t \in\left[t_{l}^{i}, t_{u}^{i}\right]:-\chi^{i}(t)+[P \gamma]^{i}(t)>0 \text { and }-\xi^{i}(t)=0\right\}
$$


and let

$$
\tilde{t}_{u}^{i} \doteq\left\{\begin{aligned}
\inf \left\{t: t \in \mathcal{A}^{i}\right\} & \text { if } \mathcal{A}^{i} \neq \emptyset \\
t_{u}^{i} & \text { otherwise. }
\end{aligned}\right.
$$

In Lemma 4.1, we first establish some properties of $\gamma^{i}$ that will be used to prove Theorem 4.2.

Lemma 4.1 For $i=1, \ldots, K$, the following properties hold.

1. $\gamma^{i}(t)=0$ for $t \in\left[0, \tilde{t}_{u}^{i}\right)$.

2. If $\mathcal{A}^{i}=\emptyset$ then $\gamma^{i}\left(\tilde{t}_{u}^{i}\right)=0$. On the other hand, if $\mathcal{A}^{i} \neq \emptyset$ then

$$
\gamma^{i}\left(\tilde{t}_{u}^{i}\right)=-\chi^{i}\left(\tilde{t}_{u}^{i}\right)+[P \gamma]^{i}\left(\tilde{t}_{u}^{i}\right) \geq 0 .
$$

Moreover, $\tilde{t}_{u}^{i} \in \operatorname{LDisc}\left(\gamma^{i}\right)$ implies $\tilde{t}_{u}^{i} \in \Phi_{-\xi^{i}}\left(\tilde{t}_{u}^{i}\right)$.

3. For $t \in\left(\tilde{t}_{u}^{i}, \infty\right)$,

$$
\gamma^{i}(t)=\sup _{s \in \Phi_{-\xi^{i}}(t)}\left[-\chi^{i}(s)+[P \gamma]^{i}(s)\right]
$$

4. For $t \in\left(\tilde{t}_{u}^{i}, \infty\right)$, if $\{t\} \neq \Phi_{-\xi^{i}}(t)$ then $t$ is a point of left increase for $\gamma^{i}$ and

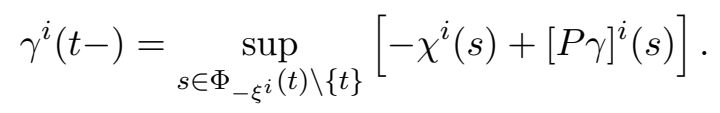

On the other hand, if $\{t\}=\Phi_{-\xi^{i}}(t)$ then

$$
\gamma^{i}(t-)=-\chi^{i}(t)+[P \gamma]^{i}(t-) \leq-\chi^{i}(t)+[P \gamma]^{i}(t)=\gamma^{i}(t) .
$$

5. For $t \in[0, \infty), t \in \operatorname{Disc}\left(\gamma^{i}\right)$ implies $t \in \Phi_{-\xi^{i}}(t)$ and

$$
\gamma^{i}(t)=\left[-\chi^{i}(t)+[P \gamma]^{i}(t)\right] \vee \gamma^{i}(t-)
$$

6. For $t \in\left[\tilde{t}_{u}^{i}, \infty\right)$, if $\Phi_{-\xi^{i}}(r) \cap[0, t]=\emptyset$ for some $r>t$ then

$$
\gamma^{i}(t+)=\gamma^{i}(t) \geq-\chi^{i}(t)+[P \gamma]^{i}(t)
$$

On the other hand, if

$$
\Phi_{-\xi^{i}}(s) \subseteq(t, s] \quad \text { for all } s>t
$$

then

$$
\gamma^{i}(t+)=-\chi^{i}(t)+[P \gamma]^{i}(t+)
$$


Proof. The first statement, and the fact that $A^{i}=\emptyset$ implies $\gamma^{i}\left(\tilde{t}_{u}^{i}\right)=0$, follow trivially from the characterization (1.17) of $\gamma^{i}$ and the definition of $\tilde{t}_{u}^{i}$. Now suppose $\mathcal{A}^{i} \neq \emptyset$. Then, by the definition of $\tilde{t}_{u}^{i}$, there must exist a sequence $\left\{s_{n}\right\} \subset\left[\tilde{t}_{u}^{i}, t_{u}^{i}\right]$ such that $s_{n} \downarrow \tilde{t}_{u}^{i}$, and for every $n \in \mathbb{N}, s_{n} \in \Phi_{-\xi^{i}}\left(s_{n}\right)\left(\right.$ since $\left.-\xi^{i}\left(s_{n}\right)=\overline{-\xi^{i}}\left(s_{n}\right)=0\right)$. By (1.17), it then follows that

$$
\gamma^{i}\left(s_{n}\right)=\sup _{s \in \Phi_{-\xi^{i}}\left(s_{n}\right)}\left[-\chi^{i}(s)+[P \gamma]^{i}(s)\right] \vee 0 \geq\left[-\chi^{i}\left(s_{n}\right)+[P \gamma]^{i}\left(s_{n}\right)\right] \vee 0>0
$$

Taking limits as $n \rightarrow \infty$ in the last expression, using the fact that $s_{n} \downarrow \tilde{t}_{u}^{i}$, along with the upper semicontinuity of $\gamma^{i}$, we see that $\gamma^{i}\left(\tilde{t}_{u}^{i}\right) \geq \gamma^{i}\left(\tilde{t}_{u}^{i}+\right) \geq 0$. Due to the continuity of $\xi$, we have $\xi^{i}\left(\tilde{t}_{u}^{i}\right)=0$, so that $\tilde{t}_{u}^{i} \in \Phi_{-\xi^{i}}\left(\tilde{t}_{u}^{i}\right)$. This in turn implies the last statement of property 2) because $\tilde{t}_{u}^{i} \in \operatorname{LDisc}\left(\gamma^{i}\right)$ holds only when $\mathcal{A}^{i} \neq \emptyset$. In addition, since $-\chi^{i}(s)+[P \gamma]^{i}(s)<0$ for $s<\tilde{t}_{u}^{i}$, this also implies that

$$
0 \leq \gamma^{i}\left(\tilde{t}_{u}^{i}\right)=\sup _{s \in \Phi_{-\xi^{i}}\left(\tilde{t}_{u}^{i}\right)}\left[-\chi^{i}(s)+[P \gamma]^{i}(s)\right] \vee 0=\left[-\chi^{i}\left(\tilde{t}_{u}^{i}\right)+[P \gamma]^{i}\left(\tilde{t}_{u}^{i}\right)\right] \vee 0,
$$

from which (4.3) follows.

For property 3$)$, we need only consider $t \in\left(\tilde{t}_{u}^{i}, t_{u}^{i}\right]$ because for $t \in\left(t_{u}^{i}, \infty\right)$, the representation (4.4) coincides with (1.17) since $\Phi_{-\xi^{i}}(t)=\Phi^{i}(t)$ by Lemma 3.8). Since $-\overline{\xi^{i}}(t)=0$ for $t \in\left(\tilde{t}_{u}^{i}, t_{u}^{i}\right]$, $\Phi_{-\xi^{i}}(\cdot)$ is monotonically non-decreasing on that interval. When combined with (1.17) and the fact that $\gamma^{i}\left(\tilde{t}_{u}^{i}\right) \geq 0$ by property 2 ), this yields the representation (4.4).

For the fourth property, fix $t>\tilde{t}_{u}^{i}$ and suppose $\{t\} \neq \Phi_{-\xi^{i}}(t)$. Since $\Phi_{-\xi^{i}}(t)$ is non-empty, this implies there exists $s<t$ such that $\overline{-\xi^{i}}(s)=\overline{-\xi^{i}}(t)$. Therefore, for every $r \in[s, t] \Phi_{-\xi^{i}}(r)=$ $\Phi_{-\xi^{i}}(t) \cap[0, u]$. As a consequence

$$
\begin{aligned}
\gamma^{i}(t-) & =\lim _{r \uparrow t} \lim _{s \in \Phi_{-\xi^{i}}(r)}\left[-\chi^{i}(s)+[P \gamma]^{i}(s)\right] \\
& =\lim _{r \uparrow t} \sup _{s \in \Phi_{-\xi^{i}}(t) \cap[0, r]}\left[-\chi^{i}(s)+[P \gamma]^{i}(s)\right] \\
& =\sup _{s \in \Phi_{-\xi^{i}}(t) \cap[0, t)}\left[-\chi^{i}(s)+[P \gamma]^{i}(s)\right]
\end{aligned}
$$

which proves (4.5). Now consider the case when $\{t\}=\Phi_{-\xi^{i}}(t)$. Let $s_{n}$ be an increasing sequence such that $s_{n} \uparrow t$, and let $u_{n} \in\left[0, s_{n}\right]$ satisfy

$$
u_{n}=\min \left\{u \in\left[0, s_{n}\right]: \overline{-\xi^{i}}(u)=\overline{-\xi^{i}}\left(s_{n}\right)\right\}
$$

We claim that then $u_{n} \uparrow t$. Indeed, since $u_{n}$ is uniformly bounded, there exists a subsequence (which we denote again by $u_{n}$ ) that converges to a limit $u_{*} \in[0, t]$. Since $\xi^{i}$ is continuous, clearly 
$u_{*} \in \Phi_{-\xi^{i}}(t)$. Since $\Phi_{-\xi^{i}}(t)=\{t\}$ by assumption, we conclude that $u_{*}=t$. Also observe that

$$
\gamma^{i}\left(s_{n}\right)=\max _{s \in \Phi_{-\xi^{i}}\left(s_{n}\right)}\left[-\chi^{i}(s)+[P \gamma]^{i}(s)\right]=\max _{s \in \Phi_{-\xi^{i}}\left(s_{n}\right) \cap\left[u_{n}, s_{n}\right]}\left[-\chi^{i}(s)+[P \gamma]^{i}(s)\right] .
$$

Take limits as $n \uparrow \infty$ on both sides of the above equality and use the fact that $u_{n} \uparrow t$ to obtain

$$
\gamma^{i}(t-)=-\chi^{i}(t)+[P \gamma]^{i}(t-) \leq-\chi^{i}(t)+[P \gamma]^{i}(t)=\gamma^{i}(t),
$$

where the inequality is a consequence of the upper semicontinuity of $[P \gamma]^{i}$ and, due to (4.4), the last equality is a trivial consequence of the fact that $\{t\}=\Phi_{-\xi^{i}}(t)$. This proves (4.6).

Due to properties 1) and 2) and the fact that $\tilde{t}_{u}^{i} \in \Phi_{-\xi^{i}}\left(\tilde{t}_{u}^{i}\right)$, it only remains to establish property 5) for $t \in\left(\tilde{t}_{u}^{i}, \infty\right)$. To do so, we shall use an argument by contradiction. Suppose $t \notin \Phi_{-\xi^{i}}(t)$. Then, since $\xi \in \mathcal{C}$, there must exist $\delta>0$ such that for $s \in[t-\delta, t+\delta]$, $-\xi^{i}(s)<\overline{-\xi^{i}}(t)$ and $\Phi_{-\xi^{i}}(s)=\Phi_{-\xi^{i}}(t)$, which in turn means that $\gamma^{i}(s)=\gamma^{i}(t)$, thus showing that $\gamma^{i}$ is continuous at $t$. Hence $t \in \operatorname{Disc}\left(\gamma^{i}\right)$ only if $t \in \Phi_{-\xi^{i}}(t)$. Along with the relations (4.4)-(4.6), this yields (4.7) and proves property 5).

In order to prove property 6$)$, first fix $t \in[0, \infty)$, and note that given a family of sets $\left\{A_{u}, u>t\right\}$ with the property that $A_{u} \subseteq(t, u]$,

$$
\lim _{u \downarrow t} \sup _{s \in A_{u}}\left[-\chi^{i}(s)+[P \gamma]^{i}(s)\right]=-\chi^{i}(t)+[P \gamma]^{i}(t+) .
$$

First suppose $\Phi_{-\xi^{i}}(r) \cap[0, t]=\emptyset$ for some $r>t$. Then, for all $u \in[t, r], \Phi_{-\xi^{i}}(u) \cap[0, t]=\Phi_{-\xi^{i}}(t)$. The representation (4.4) for $\gamma^{i}$ then shows that for every $u \in[t, r]$,

$$
\gamma^{i}(u)=\sup _{s \in \Phi_{-\xi^{i}}(t)}\left[-\chi^{i}(s)+[P \gamma]^{i}(s)\right] \vee \sup _{s \in \Phi_{-\xi^{i}}(u) \cap(t, u]}\left[-\chi^{i}(s)+[P \gamma]^{i}(s)\right] .
$$

Taking limits as $u \downarrow t$ and invoking (4.11), we obtain

$$
\gamma^{i}(t+)=\gamma^{i}(t) \vee\left[-\chi^{i}(t)+[P \gamma]^{i}(t+)\right]
$$

Together with the inequality $\gamma^{i}(t+) \leq \gamma^{i}(t)$ that holds because of the upper semicontinuity of $\gamma^{i}$, this establishes (4.8). On the other hand, if (4.9) holds then (4.10) is a direct consequence of (4.4) and (4.11) and the proof of the lemma is complete.

We now state and prove the main result of the section.

Theorem 4.2 (Discontinuities of $\gamma_{(1)}$ ) Let $\nabla \Gamma \doteq \nabla_{\chi} \Gamma(\psi)=\chi+[R \gamma]^{i}$. Then, for $i=$ $1, \ldots, K, \gamma^{i}(t)=0$ for $t \in\left[0, \tilde{t}_{u}^{i}\right)$, and the following three properties hold for every $t \in\left[t_{u}^{i}, \infty\right)$. 
1. $t \in L \operatorname{Disc}\left(\gamma^{i}\right)$ if and only if $t \in \Phi_{-\xi^{i}}(t)$ and one of the following conditions holds.

L1. $t=\tilde{t}_{u}^{i}$ and $0<-\chi\left(\tilde{t}_{u}^{i}\right)+[P \gamma]^{i}\left(\tilde{t}_{u}^{i}\right)$. In this case, $\nabla \Gamma^{i}\left(\tilde{t}_{u}^{i}-\right) \geq \nabla \Gamma^{i}\left(\tilde{t}_{u}^{i}\right)=0$.

L2. $t>\tilde{t}_{u}^{i}, \Phi_{-\xi^{i}}(t) \neq\{t\}$ and the following equality is satisfied:

$$
\sup _{s \in \Phi_{-\xi^{i}}(t) \backslash\{t\}}\left[-\chi^{i}(s)+[P \gamma]^{i}(s)\right]<-\chi^{i}(t)+[P \gamma]^{i}(t) .
$$

In this case, $\nabla \Gamma^{i}(t)=0$. Moreover, ift is not isolated in $\Phi_{-\xi^{i}}(t)$ then $t \in \operatorname{LDisc}\left([P \gamma]^{i}\right)$ and $\nabla \Gamma^{i}(t-) \geq \nabla \Gamma^{i}(t)$, while if $t \notin L D i s c\left([P \gamma]^{i}\right)$ then $\nabla \Gamma^{i}(t-)<\nabla \Gamma^{i}(t)$ and $t$ is isolated.

L3. $t>\tilde{t}_{u}^{i},\{t\}=\Phi_{-\xi^{i}}(t)$ and $t \in \operatorname{LDisc}\left([P \gamma]^{i}\right)$. In this case, $\nabla \Gamma^{i}(t-)=\nabla \Gamma^{i}(t)=0$.

2. $t \in R \operatorname{Risc}\left(\gamma^{i}\right)$ if and only if $t \in \Phi_{-\xi^{i}}(t), \Phi_{-\xi^{i}}(s) \subset(t, s]$ for all $s>t$ and one of the following conditions is satisfied.

R1. $\Phi_{-\xi^{i}}(t) \neq\{t\},[P \gamma]^{i}$ is right continuous at $t$ and

$$
\sup _{s \in \Phi_{-\xi^{i}}(t) \backslash\{t\}}\left[-\chi^{i}(s)+[P \gamma]^{i}(s)\right]>-\chi^{i}(t)+[P \gamma]^{i}(t) .
$$

In this case, $\gamma^{i}$ is left continuous at $t$ and $\nabla \Gamma^{i}(t)>\nabla \Gamma^{i}(t+)=0$.

R2. $\Phi_{-\xi^{i}}(t) \neq\{t\}$ and $t \in R \operatorname{Risc}\left([P \gamma]^{i}\right)$. In this case $\nabla \Gamma^{i}(t) \geq \nabla \Gamma^{i}(t+)=0$.

R3. $\{t\}=\Phi_{-\xi^{i}}(t)$ and $t \in R D i s c\left([P \gamma]^{i}\right)$. In this case, $\nabla \Gamma^{i}(t)=\nabla \Gamma^{i}(t+)=0$.

3. $t \in \operatorname{LDisc}\left(\gamma^{i}\right) \cap R \operatorname{Disc}\left(\gamma^{i}\right)=\operatorname{SDisc}\left(\gamma^{i}\right)$ if and only if $t \in \Phi_{-\xi^{i}}(t), \Phi_{-\xi^{i}}(s) \subset(t, s]$ for all $s>t$ and one of the following holds.

S1. $t \in R \operatorname{Risc}\left([P \gamma]^{i}\right)$, (4.12) holds and either $t \neq \Phi_{-\xi^{i}}(t)$ or $t=\tilde{t}_{u}^{i}$. In this case, $\nabla \Gamma^{i}(t)=\nabla \Gamma^{i}(t+)=0$.

S2. $\{t\}=\Phi_{-\xi^{i}}(t)$ and $t \in \operatorname{LDisc}\left([P \gamma]^{i}\right) \cap R \operatorname{Risc}\left([P \gamma]^{i}\right)$. In this case, $\nabla \Gamma^{i}(t-)=$ $\nabla \Gamma^{i}(t)=\nabla \Gamma^{i}(t+)=0$.

Proof. In the proof below, we will make repeated use of the fact that $\Gamma^{i}(t)=\chi^{i}(t)+[R \gamma]^{i}(t)=$ $\chi^{i}(t)-[P \gamma]^{i}(t)+\gamma^{i}(t)$ proved in Theorem 1.6(2), without explicit reference. The fact that $\gamma^{i}(t)=0$ for $t \in\left[0, \tilde{t}_{u}^{i}\right)$ and the fact that $\tilde{t}_{u}^{i} \in \operatorname{LDisc}\left(\gamma^{i}\right)$ if and only if $-\chi^{i}\left(\tilde{t}_{u}^{i}\right)+[P \gamma]^{i}\left(\tilde{t}_{u}^{i}\right)>0$ follows directly from properties 1 ) and 2) of Lemma 4.1. Now fix $t \in\left(\tilde{t}_{u}^{i}, \infty\right)$. Then Lemma 
4.1(5) shows that if $t \in \operatorname{LDisc}\left(\gamma^{i}\right)$ then $t \in \Phi_{-\xi^{i}}(t)$ and $\gamma^{i}(t)=\chi^{i}(t)+[P \gamma]^{i}(t)=0$, so that in this case $\nabla \Gamma^{i}(t)=0$. We consider two exhaustive sub-cases - when $t \in \Phi_{-\xi^{i}}(t) \neq\{t\}$ and $\{t\}=\Phi_{-\xi^{i}}(t)$. In the first case, the fact that (4.12) holds if and only if $t \in \operatorname{LDisc}\left(\gamma^{i}\right)$ follows from (4.5) and (4.7). If $[P \gamma]^{i}$ is continuous at $t$, then by (4.5) and the upper-semicontinuity of $\gamma^{i}$, we have

$$
\nabla \Gamma^{i}(t-)=\chi^{i}(t)-[P \gamma]^{i}(t)+\gamma^{i}(t-)<\chi^{i}(t)-[P \gamma]^{i}(t)+\gamma^{i}(t)=\nabla \Gamma^{i}(t)
$$

If $t$ is not isolated in $\Phi_{-\xi^{i}}(t)$, so that there exists a sequence $s_{n} \in \Phi_{-\xi^{i}}(t)$ with $s_{n} \uparrow t$, by (4.5), we have

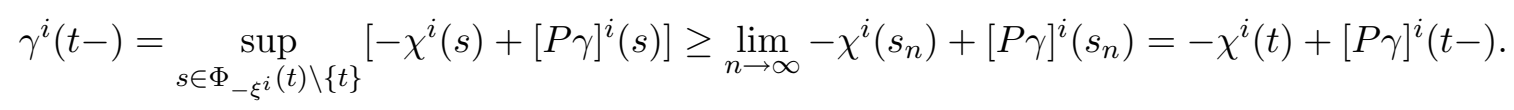

When rearranged, this shows that $\nabla \Gamma^{i}(t-) \geq 0=\nabla \Gamma^{i}(t)$, as stated in L2. Now consider the second case when $\{t\}=\Phi_{-\xi^{i}}(t)$. Then (4.6) shows that $t \in \operatorname{LDisc}\left(\gamma^{i}\right)$ if and only if $[P \gamma]^{i}$ is discontinuous at $t$, as stated in L3. This completes the proof of Theorem 1.6(1).

Now consider the right discontinuities of $\gamma^{i}$. Clearly, $R \operatorname{Disc}\left(\gamma^{i}\right) \subset\left[\tilde{t}_{u}^{i}, \infty\right)$. Properties 5) and 6) of Lemma 4.1 show that $t \in \Phi_{-\xi^{i}}(t)$ and $\Phi_{-\xi^{i}}(s) \subset(t, s]$ for all $s>t$ are necessary conditions for $t \in R \operatorname{Risc}\left(\gamma^{i}\right)$, and $\gamma^{i}(t+)=-\chi^{i}(t)+[P \gamma]^{i}(t+)$, so that $\nabla \Gamma^{i}(t+)=0$. Moreover, due to the upper semicontinuity of $[P \gamma]^{i}$, they also show that

$$
\begin{aligned}
\gamma^{i}(t) & =\left[-\chi^{i}(t)+[P \gamma]^{i}(t)\right] \vee \sup _{s \in \Phi_{-\xi^{i}}(t) \backslash\{t\}}\left[-\chi^{i}(s)+[P \gamma]^{i}(s)\right] \\
& \geq-\chi^{i}(t)+[P \gamma]^{i}(t) \\
& \geq \chi^{i}(t)+[P \gamma]^{i}(t+)=\gamma^{i}(t+)
\end{aligned}
$$

This shows that if $[P \gamma]^{i}$ is right continuous, then $\gamma^{i}(t+)=-\chi^{i}(t)+[P \gamma]^{i}(t)$ and so $t \in R D i s c\left(\gamma^{i}\right)$ if and only if $\Phi_{-\xi^{i}}(t) \neq\{t\}$ and (4.13) holds. A simple rearrangement of terms also shows that in this case $\nabla \Gamma^{i}(t)>0$ and, since the conditions (4.12) and (4.13) are mutually exclusive, it follows that $\gamma^{i}$ is left continuous at $t$. On the other hand, if $t \in R \operatorname{Risc}\left([P \gamma]^{i}\right)$, then the second inequality in (4.14) is strict and so we always have $t \in R \operatorname{Risc}\left(\gamma^{i}\right)$. Moreover, if $\gamma^{i}(t)=-\chi^{i}(t)+[P \gamma]^{i}(t)$ (as would be the case if $\{t\}=\Phi_{-\xi^{i}}(t)$ ), then $\nabla \Gamma^{i}(t)=\nabla \Gamma^{i}(t+)=0$ (as stated in R3), and otherwise $\nabla \Gamma^{i}(t)>\nabla \Gamma^{i}(t+)=0$ (from which R2 follows).

Lastly, we analyze the separated discontinuities of $\gamma^{i}$. Note that since $\gamma^{i} \in \mathcal{D}_{\text {usc }}$, it follows that $\operatorname{SDisc}\left(\gamma^{i}\right)=\operatorname{LDisc}\left(\gamma^{i}\right) \cap \operatorname{RDisc}\left(\gamma^{i}\right)$. From properties 1) and 2) of the theorem proved above, it is clear that, since the condition R1 is incompatible with both L1 and L2, the only 
ways in which this can occur is if (i) R2 and either L1 or L2 are satisfied or (ii) L3 and R3 hold, from which property 3 ) of the theorem follows immediately.

\subsection{Alternative Description of the Regimes of $(\phi, \theta)$}

In Definition 1.8, the regimes of $(\phi, \theta)$ were described in terms of properties of the solution $(\phi, \theta)$ to the ORP for an input $\psi$. On the other hand, as shown in Theorem 4.2, the analysis of the discontinuities of $\gamma$ lead naturally to conditions involving the sets $\Phi_{-\xi^{i}}(t)$ defined in Section 3.3.2. The following lemma provides a link between these two sets of conditions.

\begin{tabular}{|c||c|c|}
\hline $\begin{array}{c}\text { Physical } \\
\text { Description }\end{array}$ & $\begin{array}{c}\text { Definition } \\
\text { in terms of }(\phi, \theta)\end{array}$ & $\begin{array}{c}\text { Equivalent Condition } \\
\text { in terms of } \Phi_{-\xi^{i}}\end{array}$ \\
\hline \hline Overloaded & $\phi^{i}(t)>0$ & $t \notin \Phi_{-\xi^{i}}(t)$ \\
\hline Underloaded & $\phi^{i}(t)=0$ & $\{t\}=\Phi_{-\xi^{i}}(t)$ and \\
& $\Delta \theta^{i}(t-) \neq 0, \Delta \theta^{i}(t+) \neq 0$ & $\Phi_{-\xi^{i}}(s) \subset(t, s] \quad \forall s>t$ \\
\hline Critical & $\phi^{i}(t)=0$ and either & either $t \in \Phi_{-\xi^{i}}(t) \neq\{t\}$ or \\
& $\Delta \theta^{i}(t-)=0$ or $\Delta \theta^{i}(t+)=0$ & $\exists s>t$ such that $\Phi_{-\xi^{i}}(s) \cap[0, t] \neq \emptyset$ \\
\hline End of & $\phi^{i}(t)=0$ and $\exists \delta>0$ such that & $t \in \Phi_{-\xi^{i}}(t), \Phi_{-\xi^{i}}(t) \neq\{t\}$, \\
Overloading & $\phi^{i}(s)>0$ for $s \in(t-\delta, t]$ & $t$ is isolated in $\Phi_{-\xi^{i}}(t)$ \\
\hline Start of & $\phi^{i}(t)=0$ & $t \in \Phi_{-\xi^{i}}(t), \Phi_{-\xi^{i}}(t) \neq\{t\}$ \\
Underloading & $\Delta \theta^{i}(t-)=0$ and $\Delta \theta^{i}(t+) \neq 0$ & $\Phi_{-\xi^{i}}(s) \subset(t, s] \quad \forall s>t$ \\
\hline
\end{tabular}

Table 1: Equivalent Descriptions of the Regimes of $(\phi, \theta)$

Lemma 4.3 For $i \in\{1, \ldots, K\}$, let $\Phi_{-\xi^{i}}(t)$ be as defined by (1.14) and let $t_{l}^{i}$ be defined by (1.18). Then for $t \in\left[t_{l}^{i}, \infty\right)$, the equivalences in Table 1 are satisfied.

Proof. The lemma follows essentially from the property proved in Lemma 3.8 that for $t \geq t_{l}^{i}$, $\Phi^{i}(t)=\Phi_{-\xi^{i}}(t)$, where $\Phi^{i}$ is as defined in (1.11). The first equivalence (for the overloaded state) is then an immediate consequence of the fact that $t \in \Phi^{i}(t)$ if and only if $\phi^{i}(t)=0$. We now show that $\{t\}=\Phi_{-\xi^{i}}(t)$ if and only if $\phi^{i}(t)=0$ and $\Delta \theta^{i}(t-) \neq 0$. Indeed, $\{t\}=\Phi_{-\xi^{i}}(t)$ implies $-\xi^{i}(t)=\overline{-\xi^{i}}(t)$ and $\xi^{i}(s)<\overline{-\xi^{i}}(t)$ for all $s<t$. In turn, this holds if and only if $\theta^{i}(s)=\overline{-\xi^{i}}(s)<\overline{-\xi^{i}}(t)=\theta^{i}(t)$ for every $s<t$. Since $\theta^{i}$ is non-decreasing, this is equivalent to the condition that $\theta^{i}$ is not flat to the left of $t$. By a similar reasoning, it is easy to see that 
$\Delta \theta^{i}(t+) \neq 0$ if and only if $\Phi_{-\xi^{i}}(s) \subset(t, s]$ for all $s>t$. Combining the above equivalences, all the results of Table 1 can be obtained in a straightforward manner. The few remaining details are left to the reader.

\subsection{Proof of the Second Main Result}

We now combine the results of Theorem 4.2 and Lemma 4.3 to identify necessary conditions for discontinuities in $\nabla \Gamma$ to occur. We first establish a simple corollary of Theorem 4.2 and then present the proof of Theorem 1.10.

Lemma 4.4 For $i=1, \ldots, K$ and $t \in\left[\tilde{t}_{u}^{i}, \infty\right)$, we have the following two properties.

1. $[P \gamma]^{i}$ is left continuous at $t$ if the following condition is satisfied:

there is no critical chain preceding $i$ at time $t$

Likewise, $[P \gamma]^{i}$ is right continuous at $t$ if the following condition is satisfied.

there is no sub-critical chain preceding $i$ at time $t$

Proof. Fix $t \in[0, \infty)$ and $i \in\{1, \ldots, K\}$ and suppose (4.15) holds. Define $\mathcal{E}$ to be the class of empty chains $i=j_{0}, j_{1}, \ldots, j_{m}$ preceding $i$ at time $t$ and define

$$
M \doteq \max \left\{m: \exists j_{0} j_{1}, \ldots, j_{m} \in \mathcal{E} \text { with } t \in \operatorname{LDisc}\left(\gamma^{j_{m}}\right)\right\}
$$

Since (4.15) is satisfied, by Definition 1.9(1) of a critical chain it is clear that there are no cyclic chains in $\mathcal{E}$, so that the maximum length of an empty chain is bounded by $K$ and therefore $M$ is finite. We will argue by contradiction to show that in fact $M=0$. Suppose $M>0$ and let $j_{0} j_{1}, \ldots, j_{M} \in \mathcal{E}$ be such that $t \in \operatorname{LDisc}\left(\gamma^{j_{M}}\right)$. Let us introduce the sets $\mathcal{B} \doteq\left\{k: P_{j_{M} k}>0\right\}$ and $\tilde{B} \doteq\left\{k \in \mathcal{B}: \phi^{k}(t)=0\right\}$. By Lemma 4.3, if $k \in \tilde{B}$ then $t \notin \phi_{-\xi^{k}}(t)$ and so Theorem 4.2(1) shows that $t \notin \operatorname{LDisc}\left(\gamma^{k}\right)$. On the other hand, if $k \in B \backslash \tilde{B}$ then $j_{0} j_{1}, \ldots, j_{M}, j_{k}$ is an empty chain and the maximality of $M$ allows us to conclude once again that $t \notin \operatorname{LDisc}\left(\gamma^{j_{k}}\right)$. Together, this implies that $[P \gamma]^{j_{m}}=\sum_{k \in B} P_{j_{m} k} \gamma^{k}$ is left continuous at $t$. Theorem 4.2(1) then dictates that $i \in \operatorname{LDisc}\left(\gamma^{j_{m}}\right)$ only if $t$ is isolated in $\Phi_{-\xi_{j_{m}}}(t) \neq\{t\}$, By Lemma 4.3 this corresponds to $j_{m}$ being at the end of overloading, which in turn implies that $j_{0}, j_{1}, \ldots, j_{m}$ is a critical 
chain. Since this contradicts (4.15), we conclude that we must have $M=0$. In particular, this shows that for all $k$ with $P_{i k}>0$, either $\phi^{k}(t)=0$, in which case $j k$ is an empty chain and $M=0$ implies $t \notin \operatorname{LDisc}\left(\gamma^{k}\right)$; or $\phi^{k}(t)=0$ (which is equivalent to $t \notin \Phi_{-\xi^{k}}(t)$ ), in which case Theorem 4.2(1) shows that $t \notin L D i s c\left(\gamma^{k}\right)$. Together, this leads to the desired conclusion that $[P \gamma]^{i}=\sum_{k: P_{i k}>0} P_{i k} \gamma^{k}$ is continuous at $t$. The proof of the second assertion parallels the first, and is thus omitted.

Proof of Theorem 1.10. Fix $t \in\left(\tilde{t}_{u}^{i}, \infty\right)$. By (1.16), $\nabla \Gamma^{i}=\chi^{i}+[R \gamma]^{i}=\chi^{i}+\gamma^{i}-[P \gamma]^{i}$. Therefore $i \in \operatorname{LDisc}\left(\nabla \Gamma^{i}\right)$ only if either $t \in \operatorname{LDisc}\left([P \gamma]^{i}\right)$ or $t \in \operatorname{LDisc}\left(\gamma^{i}\right)$. First suppose that $t \in \operatorname{LDisc}\left([P \gamma]^{i}\right)$. Then, by Lemma 4.4(1), there must exist a critical chain preceding $i$, which corresponds to ( $\mathrm{Lb})$. Suppose that in addition, $i$ is overloaded, By Lemma 4.3 this means $t \in \Phi_{-\xi^{i}}(t)$, and Theorem 4.2(1) then dictates that $\gamma^{i}$ is left continuous at $t$. The inequality in (1.26) is then a direct result of the upper semicontinuity of $[P \gamma]^{i}$. Now suppose that $t \notin L \operatorname{Disc}\left([P \gamma]^{i}\right)$. Invoking Theorem 4.2(1) once again, we see (from Condition L2) that $t \in \operatorname{LDisc}\left(\nabla \Gamma^{i}\right)$ only if $t$ is isolated in $\Phi_{-\xi^{i}}(t) \neq t$, and in this case (1.25) holds. Together with Lemma 4.3, this completes the proof of Theorem 1.10(1).

The proof of the second property is analogous. If $t \in R \operatorname{Risc}\left([P \gamma]^{i}\right)$, then by Lemma 4.4(2), there must exist a sub-critical chain preceding $i$, as stated in condition $\mathrm{R}(\mathrm{b})$. If, in addition, $i$ is overloaded then $t \notin \Phi_{-\xi^{i}}(t)$ and so Theorem 4.2(2) implies that $t \notin R \operatorname{Risc}\left(\gamma^{i}\right)$. The inequality (1.28) then follows from the upper semicontinuity of $[P \gamma]^{i}$. On the other hand, if $t \notin R \operatorname{Risc}\left([P \gamma]^{i}\right)$ then for $t \in R \operatorname{Disc}\left(\nabla \Gamma^{i}\right)$ it must be that $t \in R \operatorname{Disc}\left(\gamma^{i}\right)$. By Theorem 4.2(2), this can only occur if condition R1 holds, which implies $t \in \Phi_{-\xi^{i}}(t) \neq t$ and $\Phi_{-\xi^{i}}(s) \subset(t, s]$ for all $s>t$. As shown in Lemma 4.3, this is equivalent to the statement that $i$ is at the start of underloading, which is condition $\mathrm{R}(\mathrm{a})$. The third property is implied directly by the first two, and so the proof is complete.

\section{$5 \quad$ Proof of Theorem 3.5}

In this section we prove the representation for the generalized one-dimensional derivative presented in Section 3.3.2. We first establish a lemma that will be needed for the proof of the theorem. The lemma identifies conditions under which the expression $M\left(f, g, g_{l}^{*}, g_{r}^{*}\right)$ defined in (3.17) can be expressed purely as a function of $f$ and $g$. 
Lemma 5.1 Let the sequence $\left\{g_{\varepsilon}\right\}$ satisfy the uniform boundedness and monotonicity properties stated in Theorem 3.5, and let $g_{l}^{*}$ and $g_{r}^{*}$ be defined as in Theorem 3.5. Then the following properties hold.

1. $g^{*, l} \geq g_{l}$ and $g^{*, r} \geq g_{r}$.

2. If $g_{\varepsilon}=g$ is independent of $\varepsilon$, then $g^{*, l}=g_{l}$ and $g^{*, r}=g_{r}$.

3. If $\left\{g_{\varepsilon}\right\} \subset \mathcal{C}$ then $g^{*, l}=g^{*, r}=g$.

4. If $g_{\varepsilon}$ converges to $g$ in the uniform topology, i.e. for every $N<\infty \lim _{\varepsilon \downarrow 0}\left\|g_{\varepsilon}-g\right\|_{N}=0$, then $g^{*, l}=g_{l}$ and $g^{*, r}=g_{r}$.

Proof. Fix $t \in[0, \infty)$. For every $\varepsilon>0$ choose $t_{\varepsilon} \in(t-\varepsilon, t)$ such that $\left|g_{\varepsilon}\left(t_{\varepsilon}\right)-g_{\varepsilon}(t-)\right|<\varepsilon$. Then $t_{\varepsilon} \uparrow t$ and the monotonicity of the sequence $g_{\varepsilon}$ dictates that $g\left(t_{\varepsilon}\right) \leq g_{\varepsilon}\left(t_{\varepsilon}\right)<g_{\varepsilon}(t-)+\varepsilon$. Taking limits as $\varepsilon \downarrow 0$ leads to the conclusion that $g_{l}(t)=g(t-) \leq g_{l}^{*}(t)$. An analogous argument yields the inequality $g_{r} \leq g_{r}^{*}$, thus establishing the first property. The second property is a trivial consequence of the definitions and, due to the assued monotonicity of the sequence $\left\{g_{\varepsilon}\right\}$, the third property is easily deduced from Lemma 6.4.

To prove the fourth property, for $\varepsilon \in(0,1]$ choose $t_{\varepsilon}$ such that $t_{\varepsilon} \downarrow s$ and $\left|g_{\varepsilon}\left(t_{\varepsilon}\right)-g_{\varepsilon}(s+)\right| \leq \varepsilon$. Then for any $s \in[0, \infty)$, uniform convergence of $g_{\varepsilon}$ to $g$ on the interval $[0,2 s]$ implies that given any $\delta>0$ there exists $\varepsilon_{0}>0$ such that for all $\varepsilon \in\left(0, \varepsilon_{0}\right)$ and $n \in \mathbb{N}, g\left(t_{\varepsilon}\right)-\delta \leq g_{\varepsilon}\left(t_{\varepsilon}\right) \leq g\left(t_{\varepsilon}\right)+\delta$. This in turn implies that

$$
g\left(t_{\varepsilon}\right)-\delta-\varepsilon \leq g_{\varepsilon}(s+) \leq g\left(t_{\varepsilon}\right)+\delta+\varepsilon
$$

Taking limits as $\varepsilon \downarrow 0$ we obtain the inequality

$$
g_{r}(s)-\delta=g(s+)-\delta \leq g^{*, r}(s) \leq g(s+)+\delta=g_{r}(s)+\delta,
$$

and then sending $\delta \downarrow 0$ we conclude that $g_{r}(s)=g^{*, r}(s)$. An analogous argument can be used to prove that $g_{l}=g^{*, l}$.

We now prove the characterization of the generalized one-dimensional derivative stated in Theorem 3.5.

Proof of Theorem 3.5. First note that the sequence $\left\{g_{\varepsilon}\right\}$ has a pointwise limit $g$ since for each $s \in[0, \infty)\left\{g_{\varepsilon}(s)\right\}$ is uniformly bounded and monotonically non-increasing. By the 
same token, since the left and right regularized sequences $\left\{g_{\varepsilon, l}\right\}$ and $\left\{g_{\varepsilon, r}\right\}$ inherit the uniform boundedness and monotonicity properties of $\left\{g_{\varepsilon}\right\}$, the corresponding limits $g^{*, l}$ and $g^{*, r}$ exist.

Let $t_{\ell} \doteq t_{\ell}(f)$ and $t_{u} \doteq t_{u}(f)$ be defined as in (3.18) and (3.19) respectively. Fix $t>t_{u}$, so that $\bar{f}(t)>0$ and let $L=\sup _{\varepsilon>0}\left\|g_{\varepsilon}\right\|_{t}$. By assumption $L<\infty$, and so relation (6.15) of Lemma 6.3 guarantees the existence of $\varepsilon_{0}>0$ such that $\overline{\varepsilon^{-1} f+g_{\varepsilon}}(t)>0$ for all $\varepsilon \in\left(0, \varepsilon_{0}\right)$. Hence for all $\varepsilon \in\left(0, \varepsilon_{0}\right)$, we have $\tilde{\gamma}_{\varepsilon}(t)=\overline{\varepsilon^{-1} f+g_{\varepsilon}}(t)-\overline{\varepsilon^{-1} f}(t)$. For each $\varepsilon \in\left(0, \varepsilon_{0} \wedge 1 / 8\right)$ choose $s_{\varepsilon}(t) \in[0, t]$ to satisfy

$$
\left(\varepsilon^{-1} f+g_{\varepsilon}\right)\left(s_{\varepsilon}\right) \geq \overline{\varepsilon^{-1} f+g_{\varepsilon}}(t)-8 L \varepsilon .
$$

Then, by Lemma 6.3(2) and the definition of the supremum, it is clear that $\bar{f}(t)-8 L \varepsilon \leq f\left(s_{\varepsilon}\right) \leq$ $\bar{f}(t)$. Taking limits as $\varepsilon \downarrow 0$, this yields the equality

$$
\lim _{\varepsilon \downarrow 0} f\left(s_{\varepsilon}\right)=\bar{f}(t)
$$

Moreover, we clearly also have

$$
\tilde{\gamma}_{\varepsilon}(t) \leq g_{\varepsilon}\left(s_{\varepsilon}\right)+8 L \varepsilon+\varepsilon^{-1}\left[f\left(s_{\varepsilon}\right)-\bar{f}(t)\right] \leq g_{\varepsilon}\left(s_{\varepsilon}\right)+8 L \varepsilon
$$

and therefore

$$
\limsup _{\varepsilon \downarrow 0} \tilde{\gamma}_{\varepsilon}(t) \leq \limsup _{\varepsilon \downarrow 0} g_{\varepsilon}\left(s_{\varepsilon}\right)
$$

We now show that

$$
\limsup _{\varepsilon \downarrow 0} g_{\varepsilon}\left(s_{\varepsilon}\right) \leq \tilde{\gamma}(t)
$$

Select a sequence $\left\{\varepsilon_{n}\right\}$ with $\varepsilon_{n} \downarrow 0$ such that

$$
\lim _{n \uparrow \infty} g_{\varepsilon_{n}}\left(s_{\varepsilon_{n}}\right)=\limsup _{\varepsilon \downarrow 0} g_{\varepsilon}\left(s_{\varepsilon}\right) .
$$

Since $\left\{s_{\varepsilon_{n}}\right\} \subset[0, t]$ is uniformly bounded, it can be assumed without loss of generality (by choosing a subsequence if necessary) that there exists $s_{0} \in[0, t]$ such that $\lim _{n \rightarrow \infty} s_{\varepsilon_{n}}=s_{0}$. By choosing a further subsequence if necessary, it can be assumed that either (i) $s_{\varepsilon_{n}}=s_{0}$ for all $n$ sufficiently large, or (i) does not hold and either $s_{\varepsilon_{n}} \uparrow s_{0}$ or $s_{\varepsilon_{n}} \downarrow s_{0}$ as $n \rightarrow \infty$. If (i) holds, so that $s_{\varepsilon_{n}}=s_{0}$ for all $n \in \mathbb{N}$ sufficiently large, then (5.1) implies $f\left(s_{0}\right)=\bar{f}(t)$, so that $s_{0} \in \Phi_{f}(t)$. In that case

$$
\limsup _{\varepsilon \downarrow 0} g_{\varepsilon}\left(s_{\varepsilon}\right)=\lim _{n \uparrow \infty} g_{\varepsilon_{n}}\left(s_{0}\right)=g\left(s_{0}\right) \leq \sup _{s \in \Phi_{f}(t)} g(s) \leq \tilde{\gamma}(t),
$$

and (5.3) holds. Now suppose that (i) above does not hold, but instead $s_{\varepsilon_{n}} \uparrow s_{0}$ as $n \uparrow \infty$. Then $s_{0} \in \Phi_{f}^{L}(t)$ due to (5.1). Fix $\delta>0$, and given $\varepsilon_{m}>0$ choose $N(m) \geq m$ such that for all 
$n \geq N(m), g_{\varepsilon_{m}}\left(s_{\varepsilon_{n}}\right) \leq g_{\varepsilon_{m}}\left(s_{0}-\right)+\delta$. The fact that $\left\{g_{\varepsilon_{n}}\right\}$ is a monotone non-increasing sequence as $n \uparrow \infty$ then shows that for all $n \geq N(m), g_{\varepsilon_{n}}\left(s_{\varepsilon_{n}}\right) \leq g_{\varepsilon_{m}}\left(s_{0}-\right)+\delta$. Take limits as $n \uparrow \infty$ and then $m \uparrow \infty$ to obtain

$$
\lim _{n \uparrow \infty} g_{\varepsilon_{n}}\left(s_{\varepsilon_{n}}\right) \leq \lim _{m \uparrow \infty} g_{\varepsilon_{m}}\left(s_{0}-\right)+\delta=\lim _{m \uparrow \infty} g_{\varepsilon_{m}, l}\left(s_{0}\right)+\delta=g^{*, l}\left(s_{0}\right)+\delta .
$$

Send $\delta \downarrow 0$ in the above display and use (5.4) to conclude that

$$
\limsup _{\varepsilon \downarrow 0} g_{\varepsilon}\left(s_{\varepsilon}\right) \leq g^{*, l}\left(s_{0}\right) \leq \sup _{s \in \Phi_{f}^{L}(t)} g^{*, l}(s) \leq \tilde{\gamma}(t) .
$$

Lastly, if (i) does not hold but $s_{\varepsilon_{n}} \downarrow s_{0}$ as $n \uparrow \infty$, it must be that $s_{0} \neq t$ (since $s_{\varepsilon_{n}} \in[0, t]$ ) and, due to (5.1), that $f\left(s_{0}+\right)=\bar{f}(t)$. Thus $s_{0} \in \tilde{\Phi}_{f}^{R}(t)$, and arguments similar to those given above yield the relation

$$
\limsup _{\varepsilon \downarrow 0} g_{\varepsilon}\left(s_{\varepsilon}\right) \leq \lim _{\varepsilon \downarrow 0} g_{\varepsilon}\left(s_{0}+\right)=\lim _{\varepsilon \downarrow 0} g_{\varepsilon, r}\left(s_{0}\right)=g^{*, r}\left(s_{0}\right) \leq \sup _{s \in \tilde{\Phi}_{f}^{R}(t)} g^{*, r}(s) \leq \tilde{\gamma}(t) .
$$

This establishes (5.3) which, when combined with (5.2), shows that

$$
\limsup _{\varepsilon \downarrow 0} \tilde{\gamma}_{\varepsilon}(t) \leq \tilde{\gamma}(t)
$$

In order to establish the reverse inequality (with limsup replaced by liminf) first note that for any $r \in \Phi_{f}(t)$,

$$
\tilde{\gamma}_{\varepsilon}(t) \geq \varepsilon^{-1} f(r)+g_{\varepsilon}(r)-\varepsilon^{-1} \bar{f}(t)=g_{\varepsilon}(r)
$$

First take limits as $\varepsilon \downarrow 0$ and then take the supremum over $r \in \Phi_{f}(t)$ to obtain

$$
\liminf _{\varepsilon \downarrow 0} \tilde{\gamma}_{\varepsilon}(t) \geq \sup _{r \in \Phi_{f}(t)} g(r)
$$

Now let $r \in \Phi_{f}^{L}(t)$ and for each $\varepsilon>0$ choose $r_{\varepsilon} \in[t-\varepsilon, t]$ such that

$$
\varepsilon^{-1}\left[f\left(r_{\varepsilon}\right)-\bar{f}(t)\right]>-\frac{\varepsilon}{2} \quad \text { and } \quad\left|g_{\varepsilon}\left(r_{\varepsilon}\right)-g_{\varepsilon}(r-)\right|<\frac{\varepsilon}{2}
$$

Then

$$
\tilde{\gamma}_{\varepsilon}(t) \geq \varepsilon^{-1} f\left(r_{\varepsilon}\right)+g_{\varepsilon}\left(r_{\varepsilon}\right)-\varepsilon^{-1} \bar{f}(t)>g_{\varepsilon}(r-)-\varepsilon
$$

Take limits as $\varepsilon \downarrow 0$ and then take the supremum over $r \in \Phi_{f}^{L}(t)$ to arrive at the inequality

$$
\liminf _{\varepsilon \downarrow 0} \tilde{\gamma}_{\varepsilon}(t) \geq \sup _{r \in \Phi_{f}^{L}(t)} g^{*, l}(r)
$$


Analogous arguments show that

$$
\liminf _{\varepsilon \downarrow 0} \tilde{\gamma}_{\varepsilon}(t) \geq \sup _{r \in \tilde{\Phi}_{f}^{L}(t)} g^{*, r}(r)
$$

The last two displays together with (5.6) yield the relation

$$
\liminf _{\varepsilon \downarrow 0} \tilde{\gamma}_{\varepsilon}(t) \geq \sup _{r \in \Phi_{f}^{L}(t)}\left[g^{*, l}(r)\right] \vee \sup _{r \in \Phi_{f}(t)}[g(r)] \vee \sup _{r \in \tilde{\Phi}_{f}^{R}(t)}\left[g^{*, r}(r)\right]=\tilde{\gamma}(t) .
$$

The last display along with (5.5) shows that $\lim _{\varepsilon \downarrow 0} \tilde{\gamma}_{\varepsilon}(t)=\tilde{\gamma}(t)$ for $t>t_{u}$, as desired.

For $t \in\left[t_{\ell}, t_{u}\right]$, note that $\bar{f}(t)=0$ and so $\tilde{\gamma}_{\varepsilon}(t)=\overline{\varepsilon^{-1} f+g_{\varepsilon}}(t) \vee 0$. By the same reasoning that was used for the case $t>t_{u}$, it follows that

$$
\lim _{\varepsilon \downarrow 0} \overline{\varepsilon^{-1} f+g_{\varepsilon}}(t)=\left[\sup _{s \in \Phi_{f}^{L}(t)}\left\{g^{*, l}(s)\right\} \vee \sup _{s \in \Phi_{f}(t)}\{g(s)\} \vee \sup _{s \in \tilde{\Phi}_{f}^{R}(t)}\left\{g^{*, r}(s)\right\}\right] .
$$

Taking the maximum of both sides with 0 yields the conclusion that $\lim _{\varepsilon \downarrow 0} \tilde{\gamma}_{\varepsilon}(t)=\tilde{\gamma}(t)$. Lastly, if $t<t_{\ell}$ then $\overline{\varepsilon^{-1} f}(t)<0$. Since the family $\left\{g_{\varepsilon}\right\}$ is uniformly bounded on $[0, t]$, relation $(6.16)$ of Lemma 6.3 implies that for all $\varepsilon$ sufficiently small $\overline{\varepsilon^{-1} f+g}(t)<0$. Hence for all sufficiently small $\varepsilon>0, \tilde{\gamma}_{\varepsilon}(t)=0$. Since $\tilde{\gamma}(t)=0$ for $t<t_{l}$, this completes the proof of (3.24) in Theorem 3.5 .

When $\left\{g_{\varepsilon}\right\} \subset \mathcal{C}$, by Lemma 5.1(3), $g^{*, l}=g^{*, r}=g$ and so $H\left(f, g, g^{*, l}, g^{*, r}\right)=H(f, g, g, g)=$ $H_{1}(f, g)$ and the identity (3.25) follows.

\section{Auxiliary Results}

Lemma 6.1 Any two real-valued functions $f, g$ on $[0, \infty)$ satisfy the inequality

$$
\bar{f} \vee 0-\bar{g} \vee 0 \leq \overline{f-g} \vee 0 \leq|\overline{f-g}|
$$

Moreover, if $f, g \in \mathcal{B} \mathcal{V}$ then

$$
|\bar{f} \vee 0-\bar{g} \vee 0|_{T} \leq|f-g|_{T},
$$

where $|\cdot|_{T}$ represents the total variation norm on $[0, T]$.

Proof. The first inequality in (6.7) follows from a case-by-case verification and the second inequality holds trivially. The details of the proof of (6.7) are thus left to the reader. 
For the second property, note that since $\mathcal{D}_{c, \text { lim }}$ is dense in $\mathcal{D}_{\text {lim }}$ (with respect to the topology of uniform convergence on compact sets cf., [49]), there exist sequences $\left\{f^{(n)}\right\},\left\{g^{(n)}\right\} \subset \mathcal{D}_{c}$ such that $f^{(n)} \rightarrow f$ and $g^{(n)} \rightarrow g$ as $n \rightarrow \infty$. Moreover, we clearly also have $\overline{f^{(n)}} \rightarrow \bar{f}$ and $\overline{g^{(n)}} \rightarrow \bar{g}$, and, since $f, g \in \mathcal{B V}$, it is easy to show that $\left|f^{(n)}-g^{(n)}\right|_{T} \rightarrow|f-g|_{T}$ and $\left|\overline{f^{(n)}} \vee 0-\overline{g^{(n)}} \vee 0\right|_{T} \rightarrow|\bar{f} \vee 0-\bar{g} \vee 0|_{T}$ as $n \rightarrow \infty$. Thus, to prove the second property, it suffices to establish (6.8) for $f, g \in \mathcal{D}_{c, l i m}$.

We first show that inequality (6.8) holds for $f, g \in \mathcal{D}_{c}$ of the form

$$
f=f_{0} 1_{\left[0, s_{1}\right)}+f_{1} 1_{\left[s_{1}, t\right]} \quad \text { and } \quad g=g_{0} 1_{\left[0, s_{2}\right)}+g_{1} 1_{\left[s_{2}, t\right]},
$$

where $f_{0}, f_{1}, g_{0}, g_{1} \in \mathbb{R}$ and (without loss of generality) $0 \leq s_{1} \leq s_{2} \leq t<\infty$. In this case it is clear that

$\bar{f} \vee 0-\bar{g} \vee 0=\left(f_{0} \vee 0-g_{0} \vee 0\right) 1_{\left[0, s_{1}\right)}+\left(f_{1} \vee f_{0} \vee 0-g_{0} \vee 0\right) 1_{\left[s_{1}, s_{2}\right)}+\left(f_{1} \vee f_{0} \vee 0-g_{1} \vee g_{0} \vee 0\right) 1_{\left[s_{2}, t\right]}$

and, similarly,

$$
f-g=\left(f_{0}-g_{0}\right) 1_{\left[0, s_{1}\right)}+\left(f_{1}-g_{0}\right) 1_{\left[s_{1}, s_{2}\right)}+\left(f_{1}-g_{1}\right) 1_{\left[s_{2}, t\right]} \cdot
$$

Combining this with inequality (6.7) applied to constant functions, we obtain

$$
\begin{aligned}
|\bar{f} \vee 0-\bar{g} \vee 0|_{t} & =\left|f_{1} \vee f_{0} \vee 0-f_{0} \vee 0\right|+\left|g_{1} \vee g_{0} \vee 0-g_{0} \vee 0\right| \\
& \leq\left|f_{1} \vee f_{0}-f_{0}\right|+\left|g_{1} \vee g_{0}-g_{0}\right| \\
& =\left|\left(f_{1}-f_{0}\right) \vee 0\right|+\left|\left(g_{1}-g_{0}\right) \vee 0\right| \\
& \leq\left|f_{1}-f_{0}\right|+\left|g_{1}-g_{0}\right| \\
& =|f-g|_{t},
\end{aligned}
$$

thus establishing the inequality (6.8) in this case. Similar elementary calculations can be used to show that (6.10) continues to hold for functions $f, g \in \mathcal{D}_{c, l i m} \cap \mathcal{D}_{l, r}$ that have at most one jump point in the interval $[0, t]$.

We now use the the additivity of the total variation function to show that estimates of the form (6.10) can be patched together to establish (6.8) for arbitrary functions $f, g \in \mathcal{D}_{c, l i m}$. For any real-valued function $h$ on $[0, \infty)$ and $t \in[0, \infty)$, we first define the function $h^{t}$ by

$$
h^{t}(s) \doteq h(t+s) \quad \text { for } s \in[0, \infty)
$$

and note that for $u>t, \bar{h}(u)=\overline{h^{t}}(u-t) \vee \bar{h}(t)$. Now given functions $f, g \in \mathcal{D}_{c, l i m}$, they have at most a finite number of discontinuities and hence there exists a partition $\pi=\left\{0=t_{0} \leq t_{1} \leq\right.$ 
$\left.\ldots \leq t_{k} \leq t_{m}=T\right\}$ such that for each $k=1, \ldots, m$, neither $f$ nor $g$ have any points of separated discontinuity on $\left[t_{k-1}, t_{k}\right]$ (this can clearly achieved by making including all points of separated discontinuity in the partition) and $f$ and $g$ each have at most one point of discontinuity in $\left[t_{k-1}, t_{k}\right]$. By the additivity of the total variation function, we then have

$$
|\bar{f} \vee 0-\bar{g} \vee 0|_{T}=\sum_{k=1}^{m} \bar{V}_{k} \quad \text { and } \quad|f-g|_{T}=\sum_{k=1}^{m} V_{k}
$$

where

$$
\bar{V}_{k} \doteq\left|\overline{f^{t_{k-1}}} \vee \bar{f}\left(t_{k-1}\right) \vee 0-\overline{g^{t_{k-1}}} \vee \bar{g}\left(t_{k-1}\right) \vee 0\right|_{\Delta t_{k}} \quad \text { and } \quad V_{k} \doteq\left|f^{t_{k-1}}-g^{t_{k-1}}\right|_{\Delta t_{k}}
$$

with $\Delta t_{k}=t_{k}-t_{k-1}$. Since for any function $h$ and $c \in \mathbb{R},|h+c|_{T}=|h|_{T}$, and for $a, b \in \mathbb{R}$, $a \vee b-b=(a-b) \vee 0$, we can set $c_{k}^{f}=\bar{f}\left(t_{k-1}\right) \vee 0, c_{k}^{g}=\bar{g}\left(t_{k-1}\right) \vee 0$ and rewrite $\bar{V}_{k}$ as

$$
\bar{V}_{k}=\left|\overline{f^{t_{k-1}}} \vee c_{k}^{f}-c_{k}^{f}-\overline{g^{t_{k-1}}} \vee c_{k}^{g}+c_{k}^{g}\right|_{\Delta t_{k}}=\left|\left[\overline{f^{t_{k-1}}}-c_{k}^{f}\right] \vee 0-\left[\overline{g^{t_{k-1}}}-c_{k}^{g}\right] \vee 0\right|_{\Delta t_{k}} .
$$

Observe that the functions $f^{t_{k}}(\cdot)-c_{k}^{f}$ and $g^{t_{k}}(\cdot)-c_{k}^{g}$ restricted to $\left[0, \Delta t_{k}\right]$ lie in $\mathcal{D}_{c, l i m} \cap \mathcal{D}_{l, r}$ and have at most one jump in that interval. Moreover, $\overline{f^{t_{k}}(\cdot)-c_{k}^{f}}=\overline{f^{t_{k}}}-c_{k}^{f}$ and, analogously, $\overline{g^{t_{k}}(\cdot)-c_{k}^{g}}=\overline{g^{t_{k}}}-c_{k}^{g}$. Therefore we can use (6.10) and, once again the fact that $|h+c|_{T}=|h|_{T}$, in the last expression to obtain

$\bar{V}_{k}=\left|\overline{f^{t_{k-1}}-c_{k}^{f}} \vee 0-\overline{g^{t_{k-1}}-c_{k}^{g}} \vee 0\right|_{\Delta t_{k}} \leq\left|f^{t_{k-1}}-c_{k}^{f}-g^{t_{k-1}}+c_{k}^{g}\right|_{\Delta t_{k}}=\left|f^{t_{k-1}}-g^{t_{k-1}}\right|_{\Delta t_{k}}=V_{k}$.

Summing both sides over $k=1, \ldots, m$, we obtain the desired inequality (6.8) for $f, g \in \mathcal{D}_{c, l i m}$ and the proof of the lemma is complete.

Lemma 6.2 Consider a sequence of functions $\left\{f_{n}\right\} \subset \mathcal{D}_{\lim }$ that converges pointwise to a function $f$. If for every $T<\infty \sup _{n}\left|f_{n}\right|_{T}<\infty$, then $f \in \mathcal{D}_{\lim }$.

Proof. We argue by contradiction to prove the lemma. Suppose $f \notin \mathcal{D}_{\text {lim }}$. Then there exists $t \in(0, \infty)$ such that $f$ either has no left limit or has no right limit at $t$. We assume without loss of generality that $f$ does not have a left limit at $t$ (the case when $f$ does not have a right limit follows by a similar argument). By the Cauchy condition there must exist $\delta>0$ and sequences $\left\{s_{i}\right\}$ and $\left\{s_{i}^{\prime}\right\}$ such that $s_{i} \uparrow t, s_{i}^{\prime} \uparrow t$ and for all $i, i^{\prime}$,

$$
\left|f\left(s_{i}\right)-f\left(s_{i^{\prime}}^{\prime}\right)\right| \geq 4 \delta
$$


By choosing further subsequences if necessary, we can assume that $s_{1}<s_{1}^{\prime}<s_{2}<s_{2}^{\prime}<\ldots$ without loss of generality. Since $f_{n} \rightarrow f$ pointwise, given any $m \in \mathbb{N}$ there exists $N<\infty$ such that for all $n \geq N,\left|f_{n}(s)-f(s)\right|<\delta$ for all $s \in\left\{s_{i}, s_{i}^{\prime}, i=1, \ldots, m\right\}$. Combining this inequality with (6.12) we conclude that $\left|f_{n}\left(s_{i}\right)-f_{n}\left(s_{i}^{\prime}\right)\right|>\delta$ for every $i=1, \ldots, m$ and $n \geq N$, and hence that

$$
\left|f_{n}\right|_{t} \geq \sum_{i=1}^{m}\left|f_{n}\left(s_{i}\right)-f_{n}\left(s_{i}^{\prime}\right)\right| \geq m \delta .
$$

Taking the supremum over all $n$, and then letting $m$ go to infinity, we conclude that $\sup _{n}\left|f_{n}\right|_{t}=$ $\infty$, which leads to a contradiction. Thus it must be that $f \in \mathcal{D}_{\lim }$.

Lemma 6.3 Suppose $f \in \mathcal{D}_{\lim }$ and $\left\{g_{\varepsilon}, \varepsilon>0\right\} \subseteq \mathcal{D}_{\text {lim }}$ satisfies

$$
L_{N} \doteq \sup _{\varepsilon>0}\left\|g_{\varepsilon}\right\|_{N}<\infty \quad \text { for every } N \in[0, \infty)
$$

Then the following properties hold for any $t \in[0, \infty)$.

1. There exists $\varepsilon_{0}>0$ such that for all $\varepsilon \in\left(0, \varepsilon_{0}\right)$

$$
\bar{f}(t)<0 \quad \Rightarrow \quad \overline{\varepsilon^{-1} f+g_{\varepsilon}}(t)<0
$$

and likewise

$$
\bar{f}(t)>0 \Rightarrow \overline{\varepsilon^{-1} f+g_{\varepsilon}}(t)>0 .
$$

2. Given $\delta \in\left(0, L_{t}\right)$, if

$$
\overline{\varepsilon^{-1} f+g_{\varepsilon}}(t) \leq \varepsilon^{-1} f(s)+g_{\varepsilon}(s)+\delta
$$

for some $s \in[0, t]$ and $\varepsilon \in\left(0, \delta / 6 L_{t}\right)$, then

$$
\bar{f}(t) \leq f(s)+\delta
$$

Proof. Fix $t \in[0, \infty)$, let $L \doteq L_{t}$ and choose $\varepsilon_{0}=|\bar{f}(t)| / 2 L$. If $\bar{f}(t)<0$, then for $\varepsilon \in\left(0, \varepsilon_{0}\right)$

$$
\overline{\varepsilon^{-1} f+g_{\varepsilon}} \leq \varepsilon^{-1} \bar{f}(t)+\bar{g}_{\varepsilon}(t) \leq-2 L+L<0
$$

which establishes (6.14). A similar argument establishes (6.15).

We now argue by contradiction to establish the second property. Suppose there exists $\varepsilon<$ $\delta / 6 L_{t}$ and $s \in[0, t]$ that satisfies (6.16), but for which $f(s)<\bar{f}(t)-\delta$. Choose $\tilde{s} \in[0, t]$ such 
that $f(\tilde{s})>\bar{f}(t)-\delta / 2$. Then the last two inequalities together show that $f(\tilde{s})>f(s)+\delta / 2$ which, along with (6.16), the fact that $\delta<L_{t}$ and $\varepsilon<\delta / 6 L_{t}$, implies that

$$
\begin{aligned}
\varepsilon^{-1} f(\tilde{s})+g_{\varepsilon}(\tilde{s})-\overline{\varepsilon^{-1} f+g_{\varepsilon}}(t) & \geq \varepsilon^{-1} f(\tilde{s})+g_{\varepsilon}(\tilde{s})-\left(\varepsilon^{-1} f(s)+g_{\varepsilon}(s)\right)-\delta \\
& \geq \varepsilon^{-1}[f(\tilde{s})-f(s)]-2 L_{t}-\delta \\
& >\frac{\varepsilon^{-1} \delta}{2}-3 L_{t}>0
\end{aligned}
$$

which contradicts the definition of the supremum since $\tilde{s} \in[0, t]$.

Lemma 6.4 Consider a family of left (respectively right) continuous functions $\left\{g_{\varepsilon}\right\}$ that converges pointwise monotonically down to a function $g \in \mathcal{D}_{\lim }$ as $\varepsilon \downarrow 0$. If $s$ is a point of left (respectively right) continuity for $g$, then given any sequence $s_{\varepsilon} \uparrow s$ (respectively $s_{\varepsilon} \downarrow s$ )

$$
\lim _{\varepsilon \downarrow 0} g_{\varepsilon}\left(s_{\varepsilon}\right)=g(s)
$$

Proof. Fix $s \in[0, \infty)$. Given any $\delta>0$, the pointwise convergence of $g_{\kappa}$ shows that there exists $\kappa_{0}>0$ such that for all $\kappa \in\left(0, \kappa_{0}\right),\left|g_{\kappa}(s)-g(s)\right|<\delta / 2$. Likewise, given any $\kappa>0$, since either $g_{\kappa}$ is left continuous and $s_{\varepsilon} \uparrow s$, or $g_{\kappa}$ is right continuous and $s_{\varepsilon} \downarrow 0$, there exists $\varepsilon_{0}(\kappa)<\kappa$ such that for all $\varepsilon<\varepsilon_{0}(\kappa),\left|g_{\kappa}\left(s_{\varepsilon}\right)-g_{\kappa}(s)\right|<\delta / 2$. Together, the last two inequalities show that given any $\delta>0$ there exists $\kappa_{0}>0$ such that for all $\kappa<\kappa_{0}$ and $\varepsilon<\varepsilon_{0}(\kappa),\left|g_{\kappa}\left(s_{\varepsilon}\right)-g(s)\right|<\delta$. When combined with the fact that $\varepsilon<\varepsilon_{0}(\kappa)<\kappa$ and $g_{\varepsilon}$ converges pointwise monotonically down to $g$, this implies that

$$
g\left(s_{\varepsilon}\right) \leq g_{\varepsilon}\left(s_{\varepsilon}\right) \leq g_{\kappa}\left(s_{\varepsilon}\right) \leq g(s)+\delta .
$$

Taking limits as $\varepsilon \downarrow 0$ and using the left continuity of $g$ and the fact that $s_{\varepsilon} \uparrow s$ (or the right continuity of $g$ and the fact that $s_{\varepsilon} \downarrow s$ ), one concludes that

$$
g(s) \leq \liminf _{\varepsilon \downarrow 0} g_{\varepsilon}\left(s_{\varepsilon}\right) \leq \limsup _{\varepsilon \downarrow 0} g_{\varepsilon}\left(s_{\varepsilon}\right) \leq g(s)+\delta .
$$

Sending $\delta \downarrow 0$ concludes the proof of the lemma.

Acknowledgements. The authors would like to thank Ward Whitt for his valuable comments, as well as for his encouragement throughout the course of this work. The authors are also grateful to Lillian Bluestein for her help in typing up parts of this manuscript. The second author would also like to thank Rami Atar, Avi Mandelbaum, Adam Shwartz and Ofer Zeitouni for an 
invitation to the Technion, which provided the leisure necessary to complete this paper. Finally, the authors are grateful to anonymous referees for many constructive comments that helped improve the exposition of the paper.

\section{References}

[1] S. Asmussen and H. Thorisson. 1987. A Markov chain approach to periodic queues. J. Appl. Probab., 24:215-225.

[2] N. Bambos and J. Walrand. 1989. On queues with periodic inputs. J. Appl. Probab., $26: 381-389$.

[3] A. Bernard and A. El Kharroubi. 1991. Regulation de processus dans le premier orthant de $\mathbb{R}^{n}$. Stoch. and Stoch. Rep., 34:149-167.

[4] A. Berman and R. Plemmons. 1994. Nonnegtaive Matrices in the Mathematical Sciences. SIAM, New York.

[5] P. Billingsley. 1968. Convergence of Probability Measures. John Wiley, New York.

[6] M. Bramson and J. Dai. 2001. Heavy traffic limits for some queueing networks. Ann. of App. Prob., 11:49-90.

[7] H. Chen and A. Mandelbaum. 1991. Discrete flow networks: Bottleneck analysis and fluid approximations. Math. Oper. Res., 16:408-446.

[8] H. Chen and A. Mandelbaum. 1991. Discrete flow networks: Diffusion approximations and bottlenecks Ann. Prob., 19:1463-1519.

[9] P. Dupuis and H. Ishii. 1991. On Lipschitz continuity of the solution mapping to the Skorokhod problem, with applications. Stochastics, 35:31-62.

[10] P. Dupuis and K. Ramanan. 1999. Convex duality and the Skorokhod Problem, I, II. Prob. Theor. and Rel. Fields, 115:153-195 (I); 197-236 (II).

[11] M. Gerla and L. Kleinrock. 1980. Flow control: A comparative survey. IEEE Trans. Commun. vol. COM-29, 4:553-574. 
[12] P. Glynn. 1998. Strong approximations in queueing theory. Asymptotic Methods in Probability and Statistics: A Volume in Honour of Mikols Csorgo, B. Szyszkowicz, ed., Elsevier Press, Netherlands, 135-150.

[13] S. Halfin and W. Whitt. 1981. Heavy-traffic limit theorems for queues with many exponential servers. Oper. Res., 29:567-588.

[14] J.M. Harrison. 1985. Brownian Motion and Stochastic Flow Systems. Wiley, New York.

[15] J. M. Harrison and A. J. Lemoine. 1977. Limit theorems for periodic queues, J. Appl. Prob., 14:566-576.

[16] J.M. Harrison and M.I. Reiman. 1981. Reflected Brownian motion on an orthant. Ann. of Prob., 9:302-308.

[17] D. P. Heyman and W. Whitt. 1984. The asymptotic behaviour of queues with time-varying arrival rates. J. Appl. Prob., 21:143-156.

[18] 2003. P. Jelenkovic, A. Mandelbaum and P. Momcilovic. Heavy traffic limits for queues with many deterministic servers. To appear in Queueing Syst..

[19] J. B. Keller. 1982. Time-dependent queues. SIAM Rev., 24, 401-412.

[20] N. Gans, G. Koole and A. Mandelbaum. 2003. Telephone call centers: Tutorial, review and research projects. Invited review paper by Manufacturing and Service Operations Management, $\mathbf{5}$.

[21] H. Kushner. 2001. Heavy Traffic Analysis of Controlled Queueing and Communication Networks. Springer-Verlag, New York.

[22] A. J. Lemoine. 1989. Waiting time and workload in queues with periodic Poisson input. J. Appl. Prob., 26:390-397.

[23] W. P. Lovegrove, J. L. Hammond and D. Tipper. 1990. Simulation methods for studying nonstationary behavior of computer networks. IEEE Journal of Sel. Areas in Comm., 9:1696-1708.

[24] A. Mandelbaum. 1989. The dynamic complementarity problem. Unpublished manuscript. 
[25] A. Mandelbaum and W. Massey. 1995. Strong approximations for time-dependent queues. Math. of Oper. Res., 20:33-63.

[26] A. Mandelbaum, W. Massey and M. Reiman. 1998. Strong approximations for Markovian service networks. Queueing Syst. 30:149-201.

[27] A. Mandelbaum and G. Pats. 1998. State-dependent stochastic networks. PartI: Approximations and applications with continuous diffusion limits. Ann. of App. Prob., 2:569-646.

[28] A. Mandelbaum and K. Ramanan. Functional central limit theorems for non-stationary queueing networks. In preparation.

[29] A. Mandelbaum and K. Ramanan. Directional derivatives of the QED map. In preparation.

[30] A. Mandelbaum and L. Van der Heyden. 1987. Complementarity and reflection. Unpublished work.

[31] W. Massey. 1981. Nonstationary queues. Ph.D. Thesis, Stanford University, CA.

[32] W. Massey. 1985. Asymptotic analysis of the time dependent M/M/1 Queue. Math. of Oper. Res., 10:305-327.

[33] W. Massey and W. Whitt. 1993. Networks of infinite-server queues with nonstationary Poisson input. Queueing Systems, 13:183-250.

[34] G. F. Newell. 1968. Queues with time-dependent arrival rates I, II, III. J. Appl. Prob. 5:436-451 (I); 570-590 (II); 591-606 (III).

[35] G. F. Newell. 1982. Applications of Queueing Theory. Chapman and Hall, Second Edition.

[36] J. L. Pomerade. 1976. A unified approach via graphs to Skorokhod's topologies on the function space D. Ph.D. Thesis, Dept. of Statistics, Yale University.

[37] W. P. Peterson. 1991. Diffusion approximations for networks of queues with multiple customer types. Math. of Oper. Res., 9:90-118.

[38] A. Puhalskii and M. Reiman. 2000. The multiclass GI/PH/N queue in the Halfin-Whitt regime. Adv. in App. Prob. 32, 564-595. 
[39] K. Ramanan. Functional Central Limits for Non-stationary Queueing Networks In preparation.

[40] K. Ramanan and M. Reiman. 2003. Fluid and heavy traffic diffusion limits for a generalized processor sharing model. Ann. of App. Prob., 13 1:100-139.

[41] M.I. Reiman. 1984. Open queueing networks in heavy traffic. Math. of Oper. Res., 9:441458.

[42] T. Rolski. 1981. Queues with non-stationary input stream: Ross' conjecture. Adv. Appl. Prob., 13:608-618.

[43] T. Rolski. 1990. Queues with non-stationary inputs. Queueing Systems, 5:113-130.

[44] H. L. Royden. 1989. Real Analysis, Third Edition. Macmillan Publishing Co., New York.

[45] A. V. Skorokhod. 1956. Limit theorems for stochastic processes. Theor. Prob. and its Appl., $1: 261-290$.

[46] A. V. Skorokhod. 1961. Stochastic equations for diffusions in a bounded region. Theor. of Prob. and its Appl., 6:264-274.

[47] D. R. Smart. 1974. Fixed Point Theorems. Cambridge University Press, Great Britain.

[48] A. F. Veinott Jr. 1969. Discrete dynamic programming with sensitive discount optimality criteria. Ann. Math. Statist., 40:1635-1660.

[49] W. Whitt. 2002. An Introduction to Stochastic-Process Limits and Their Application to Queues. Springer-Verlag, New York.

[50] W. Whitt. 2002. An Introduction to Stochastic-Process Limits and Their Application to Queues. Internet Supplement, http://www.columbia.edu/ ww2040/supplement.html.

[51] R. Williams. 1998. Reflecting diffusions and queueing networks. Proceedings of the ICM. 

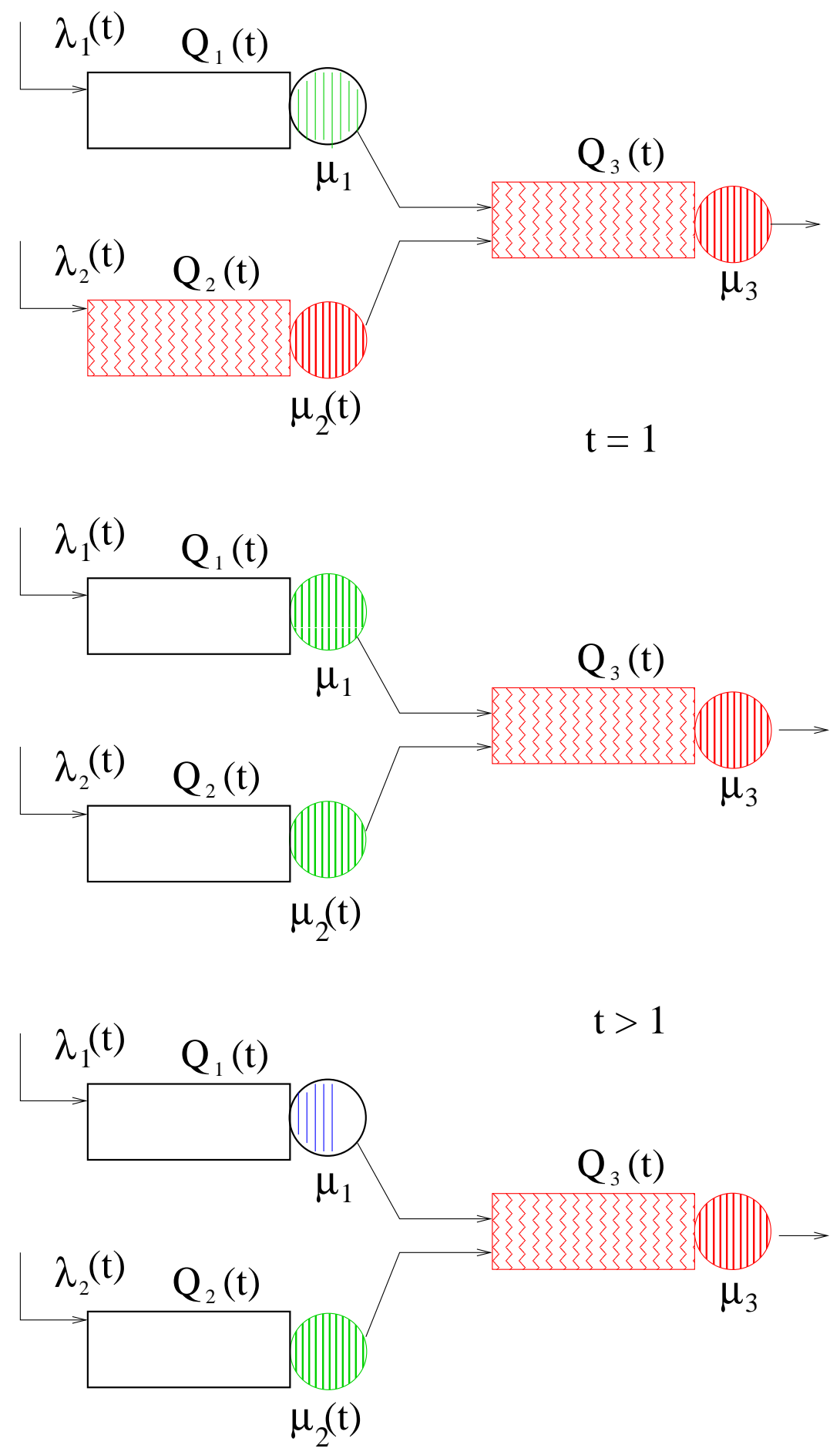

Figure 3: A queueing network with a merge and time-varying arrival and sevice rates giving rise to a separated discontinuity in the directional derivative at $t=1$ 


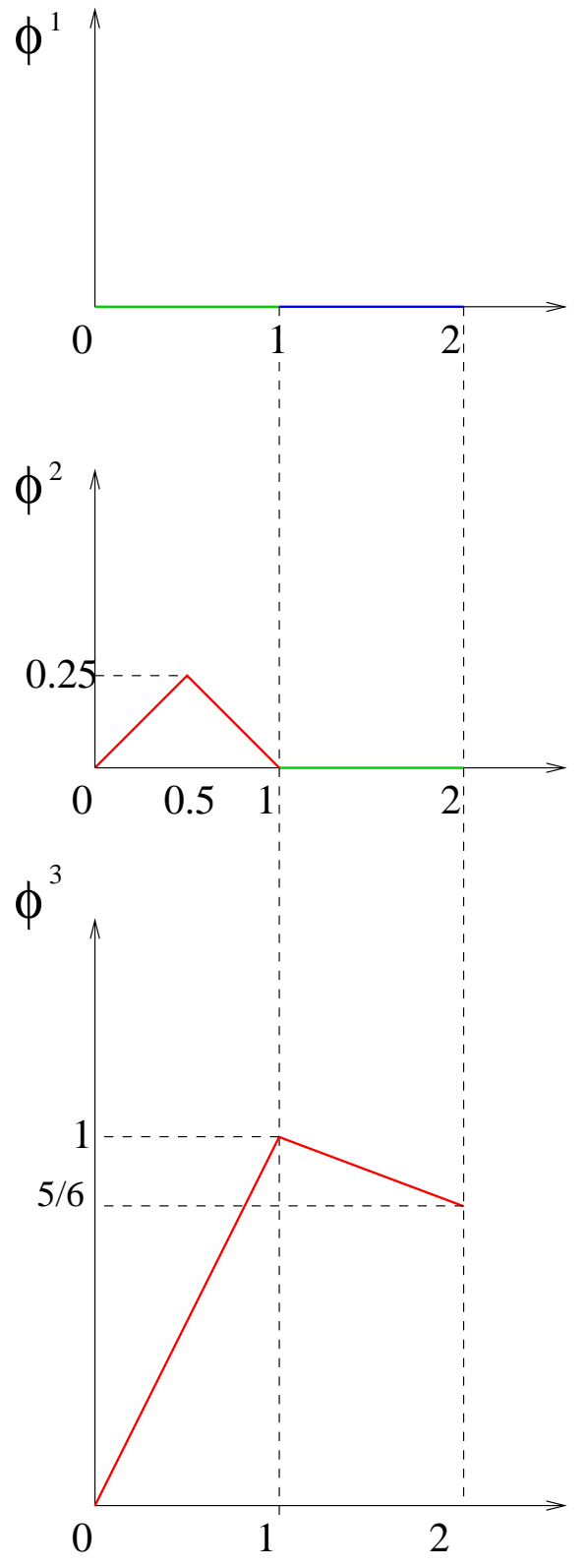

Figure 4: The fluid limit of the non-stationary merge queueing network 